University of Nebraska - Lincoln

DigitalCommons@University of Nebraska - Lincoln

$11-2011$

\title{
A Mild One-Pot Conversion of Alkenes into Amines through Tandem Ozonolysis and Reductive Amination
}

\author{
ShivaKumar Kyasa \\ University of Nebraska-Lincoln \\ Thomas J. Fisher \\ University of Nebraska-Lincoln, \\ Patrick Dussault \\ University of Nebraska-Lincoln, pdussault1@unl.edu
}

Follow this and additional works at: https://digitalcommons.unl.edu/chemistrydussault

Part of the Chemistry Commons

Kyasa, ShivaKumar; Fisher, Thomas J.; and Dussault, Patrick, "A Mild One-Pot Conversion of Alkenes into Amines through Tandem Ozonolysis and Reductive Amination" (2011). Patrick Dussault Publications. 13. https://digitalcommons.unl.edu/chemistrydussault/13

This Article is brought to you for free and open access by the Published Research - Department of Chemistry at DigitalCommons@University of Nebraska - Lincoln. It has been accepted for inclusion in Patrick Dussault Publications by an authorized administrator of DigitalCommons@University of Nebraska - Lincoln. 
Published in Synthesis 21 (2011), pp. 3475-3481; doi: 10.1055/s-0030-1260244

Copyright ( 2011 Georg Thieme Verlag, Stuttgart \& New York. Used by permission.

Submitted June 30, 2011; published online September 27, 2011; in print November 2, 2011.

\title{
A Mild One-Pot Conversion of Alkenes into Amines through Tandem Ozonolysis and Reductive Amination
}

\author{
ShivaKumar Kyasa, ${ }^{1}$ Thomas J. Fisher, ${ }^{1}$ and Patrick H. Dussault \\ Department of Chemistry, University of Nebraska-Lincoln, Lincoln, NE 68588-0304, USA \\ Corresponding author - P. Dussault, fax 402 472-9402, email pdussault1@unl.edu
}

\begin{abstract}
The selective reduction of hydroperoxyacetals to aldehydes by sodium triacetoxyborohydride provides the basis for a mild one-pot synthesis of amines from alkenes.
\end{abstract}

Keywords: ozonolysis, hydroperoxyacetal, reductive amination, amine, sodium triacetoxyborohydride

Reductive amination is a widely used transformation in organic synthesis. ${ }^{2}$ The carbonyl precursors are often prepared from alkenes through ozonolysis followed by reduction of the ozonide or peroxide intermediates. ${ }^{3,4}$ In the course of investigations into new transformations based upon fragmentation of ozonolysis intermediates, ${ }^{5}$ we became interested in the development of a mild method for a one-pot conversion of alkenes to amines. Reductive amination is frequently achieved through the reaction of carbonyls and amines in the presence of deactivated boron hydrides, $2 \mathrm{~b}, 6,7$ and $\mathrm{NaCNBH}_{3}$-promoted reductive amination has been applied in tandem with ozonolysis. ${ }^{8}$ However, $\mathrm{NaCNBH}_{3}$ reduces ozonides (1,2,4-trioxolanes) very slowly, ${ }^{9}$ and some of the reported transformations may actually involve amine-promoted $\mathrm{E}_{1} \mathrm{cb}$ fragmentation of terminal ozonides. ${ }^{10}, 11$ We investigated the reaction of $\mathrm{NaCNBH}_{3}$ with more reactive hydroperoxyacetals, but observed significant formation of alcohols. Our attention was therefore turned to the less reactive triacetoxyborohydride, which has been investigated little in ozonolysis/amination sequences. ${ }^{12,}{ }^{13}$ We now report that $\mathrm{NaBH}(\mathrm{OAc})_{3}$ allows rapid and efficient reduction of ozonolysis-derived hydroperoxyacetals to aldehydes, enabling a mild and convenient one-pot synthesis of amines from alkenes based upon ozonolysis and reductive amination.

The starting hydroperoxyacetals were readily available through ozonolysis of precursor alkenes or enol ethers in 3\% $\mathrm{MeOH}-\mathrm{CH}_{2} \mathrm{Cl}_{2}$ (Table 1). ${ }^{14,15}$

Reduction conditions were initially screened on hydroperoxyacetal $1 b$ using ${ }^{1} \mathrm{H}$ NMR of crude reaction mixtures to monitor the ratio of starting hydroperoxyacetal, aldehyde (nonanal), and the alcohol (nonanol) derived from overreduction. It was found that $\mathrm{NaBH}(\mathrm{OAc})_{3}$ offered high selectivity for aldehyde formation in several different solvent systems $\left(\mathrm{CH}_{2} \mathrm{Cl}_{2}, \mathrm{DCE}\right.$, THF) and under several sets of conditions. Performing the reduction with one equivalent of $\mathrm{NaBH}(\mathrm{OAc})_{3}$ proved optimal; and excess of reducing reagent (1.5-3 equiv) led to slow overreduction of the aldehyde. In contrast, the use of the more reactive $\mathrm{NaCNBH}_{3}$ in stoichiometric amounts cleanly generated alcohol, while a mixture of alcohol and starting material was observed even with only 0.33 equivalent of reagent.
Table 1. Preparation of Hydroperoxyacetals

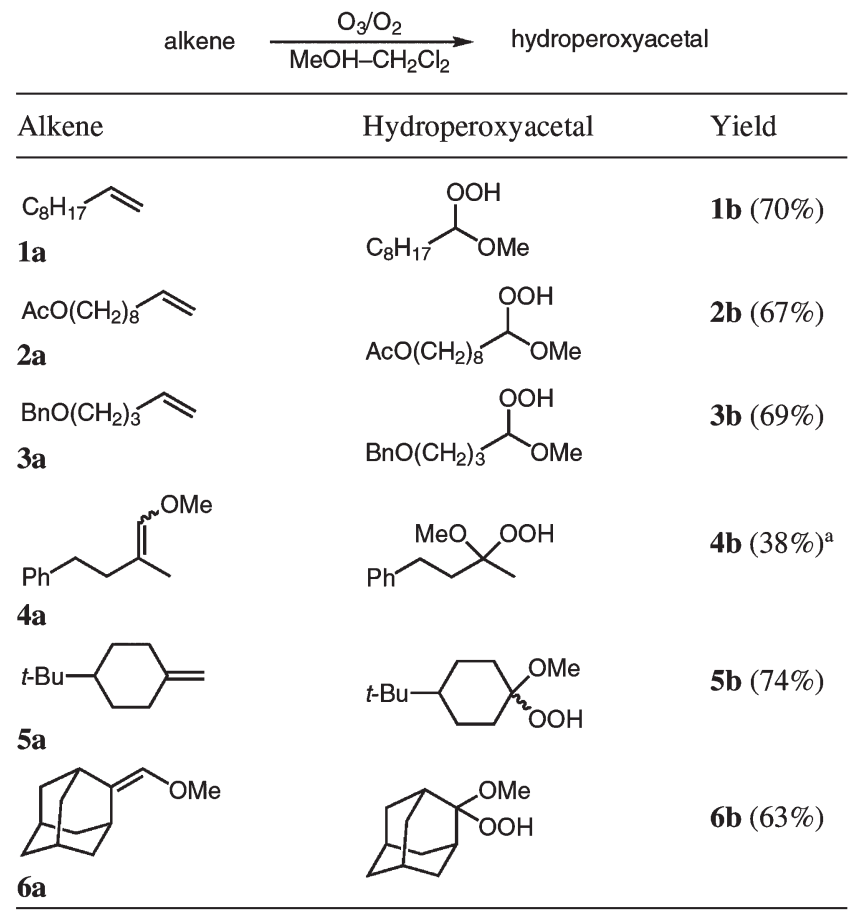

a Partial decomposition upon chromatography.

Reduction with $\mathrm{NaBH}(\mathrm{OAc})_{3}$ was next applied to a wider group of hydroperoxyacetals (Table 2). Hydroperoxyacetals reacted faster $(2 \mathrm{~h})$ than hydroperoxyketals (5-8 h). In both cases, a high yield of the carbonyl was obtained with little or no overreduction.

Reductive amination was easily conducted through reduction of the hydroperoxyacetal with $\mathrm{NaBH}(\mathrm{OAc})_{3}$ (1 equiv), followed by addition of amine and additional hydride (Scheme 1). The reactions were found to give good yields of monoalkylated amines. For the amination of aldehyde $2 b$ with benzylamine, $8 \%$ of the tertiary amine derived from overalkylation was isolated, in addition to the expected secondary amine $2 f$.

Finally, it was found that ozonolysis and reductive amination could be easily combined into a one-pot transformation 
Table 2. Reduction of Hydroperoxyacetals with $\mathrm{NaBH}(\mathrm{OAc})_{3}$

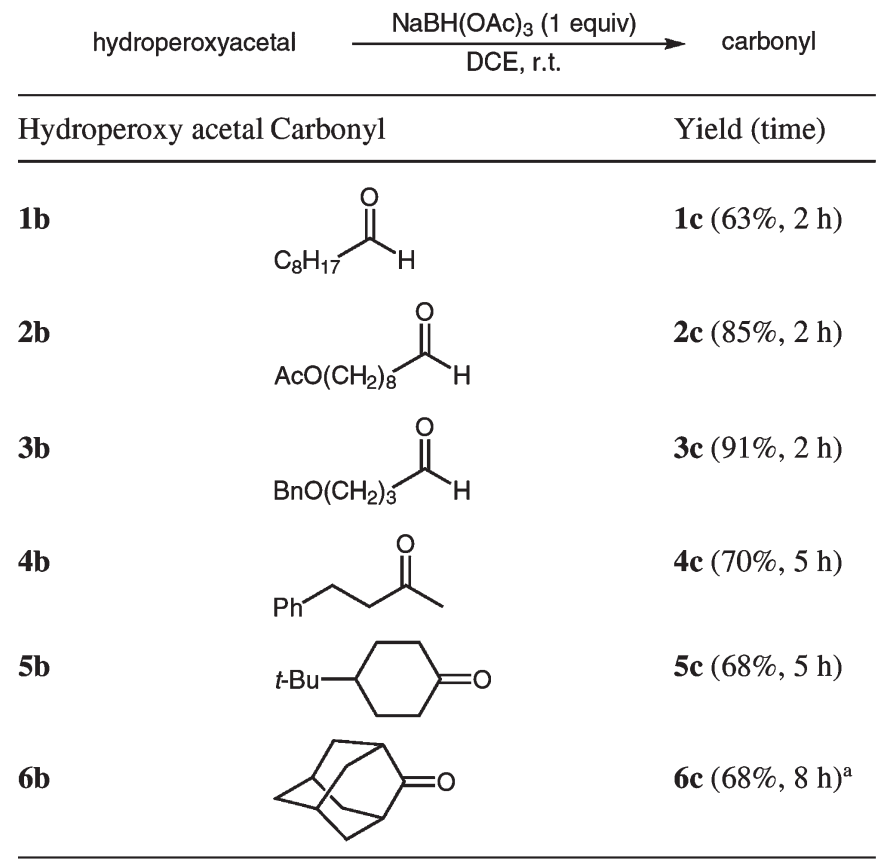

a Based upon $15 \%$ recovered starting material.

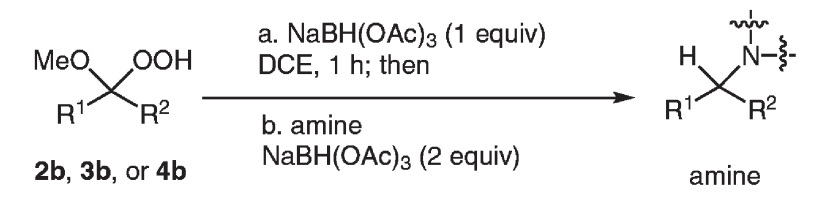

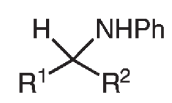
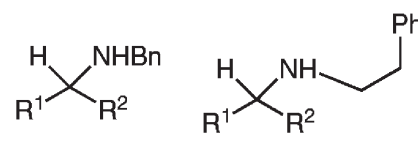

2d: $85 \%$

3d: $89 \%$

4d: $45 \%$ 2f: $67 \%^{\mathrm{a}}$

3f: $63 \%$

4f: $75 \%$

$\begin{array}{ll}\text { 2g: } & 63 \% \\ \text { 3g: } & 65 \% \\ \mathbf{4 g :} & 52 \%\end{array}$

2d-g: $\mathrm{R}^{1}=\mathrm{AcO}\left(\mathrm{CH}_{2}\right)_{8}: \mathrm{R}^{2}=\mathrm{H}$

3d-g: $\mathrm{R}^{1}=\mathrm{BnO}\left(\mathrm{CH}_{2}\right)_{3} ; \mathrm{R}^{2}=\mathrm{H}$

4d-g: $\mathrm{R}^{1}=\mathrm{Ph}\left(\mathrm{CH}_{2}\right)_{2} ; \mathrm{R}^{2}=\mathrm{Me}$

a $8 \%$ of $3^{\circ}$ amine was also isolated.

Scheme 1. Stepwise reduction-reductive amination

(Table 3). Ozonolysis of an alkene substrate was conducted in methanol- $\mathrm{CH}_{2} \mathrm{Cl}_{2}$ until the alkene could no longer be detected (TLC). The crude reaction mixture was then submitted to one of two protocols. In the first (method A), addition of acetoxyborohydride (1 equiv) was followed, after 45 minutes to 1 hour, by addition of amine and additional acetoxyborohydride. More conveniently (method B), the crude ozonolysis product was treated with acetoxyborohydride (3 equiv) and amine (1-2 equiv). Similar yields were obtained from the two sequences. Under these conditions, cyclic alkenes un-
Table 3. Stepwise versus One-Pot Reductive Amination

$$
\begin{aligned}
& \mathrm{O}_{3}, \mathrm{CH}_{2} \mathrm{Cl}_{2}-\mathrm{MeOH} \text {; then: } \\
& \text { A) } \mathrm{NaBH}(\mathrm{OAc})_{3} \text {; then reactant } \\
& \text { amine, additional } \mathrm{NaBH}(\mathrm{OAc})_{3}
\end{aligned}
$$

Alkene Reactant amine Method (yield) Product amine

○ी $\mathrm{PhCH}_{2} \mathrm{NH}_{2} \quad \mathrm{~A}(57 \%)$

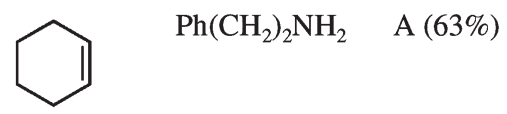

$\begin{array}{ll}\text { 3a } & \begin{array}{l}\text { morpholine } \\ \mathrm{PhCH}_{2} \mathrm{NH}_{2}\end{array} \\ & \mathrm{~A}(65 \%)\end{array}$

7

3d<smiles>c1ccc(CN2CCCCC2)cc1</smiles>

9

$\begin{array}{ll}\mathrm{Ph}\left(\mathrm{CH}_{2}\right)_{2} \mathrm{NH}_{2} & \mathrm{~A}(72 \%) \\ & \mathrm{B}(62 \%)\end{array}$

2a morpholine $\quad$ B $(66 \%) \quad 2 d$

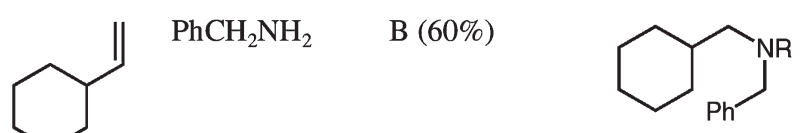

11a $\mathrm{R}=\mathrm{H} 50 \%$

11b $\mathrm{R}=\mathrm{Me} 10 \%$<smiles>C1=CC2CCC1C2</smiles>

$\mathrm{Ph}\left(\mathrm{CH}_{2}\right)_{2} \mathrm{NH}_{2}$

A $(65 \%)$

B $(65 \%)$

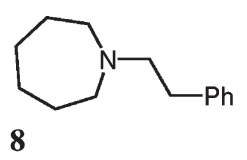

12

dergo a tandem oxidation/reduction amination sequence to provide a variety of heterocyclic amines with an average yield of $\sim 90 \%$ per reaction. In some cases, condensation of the product secondary amine with residual formaldehyde, a by-product of the ozonolysis of terminal olefins, gave rise to a tertiary amine (e.g., 11b).

A very similar protocol can be applied to the synthesis of hydrazones, hydrazines, and diazenes. Ozonolysis of $4 a$, followed by treatment of the crude hydroperoxyacetal with phenylhydrazine and $\mathrm{NaBH}(\mathrm{OAc})_{3}$, cleanly furnished a phenylhydrazone (TLC). Addition of $\mathrm{NaCNBH}_{3}$ resulted in the formation of the phenylhydrazide, which underwent rapid air oxidation during isolation to furnish the diazene as the isolated material in 57\% yield (Scheme 2). 


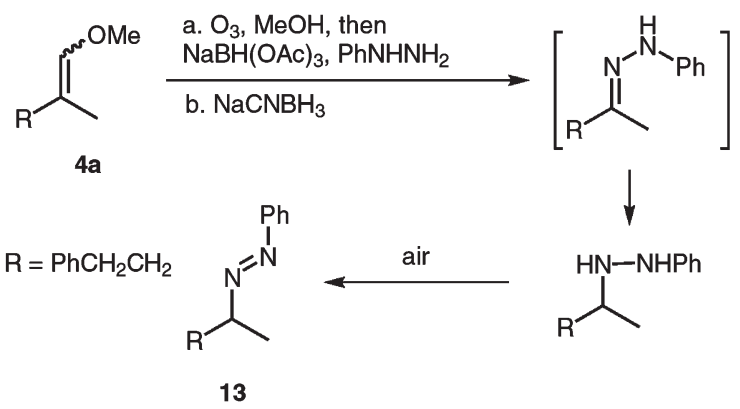

Scheme 2

While there are many procedures for conversion of alkenes to amines, most require separate oxidation and amination steps, each involving isolation and purification. The procedure reported here, which allows a one-pot conversion of alkenes to a variety of amines while avoiding any accumulation of peroxide intermediates, may be of particular use for preparation of an array of amines from a common precursor, or in procedures limited by concerns with isolation or handling of peroxide intermediates. Utilization of $\mathrm{NaBH}(\mathrm{OAc})_{3}$ in place of $\mathrm{NaCNBH}_{3}$ provides a more economical, safe, and selective protocol for the one-pot tandem ozonolysis/reductive amination sequence for the conversion of alkenes to amines. The speed and selectivity of the reduction of the hydroperoxyacetals suggests the formation and decomposition of a peroxyborate, $\mathrm{ROOBH}(\mathrm{OAc})_{2}$.

All reagents were used as received from commercial vendors, with the exception of $\mathrm{CH}_{2} \mathrm{Cl}_{2}$, which was distilled from $\mathrm{CaH}_{2}$, and THF, which was distilled from $\mathrm{Na}$ /benzophenone. TLC was performed on $0.25 \mathrm{~mm}$ hard-layer silica G plates; developed plates were visualized with a hand-held UV lamp or by staining: $1 \% \mathrm{Ce}\left(\mathrm{SO}_{4}\right)_{2}$ and $10 \%\left(\mathrm{NH}_{4}\right)_{2} \mathrm{MoO}_{4}$ in $10 \% \mathrm{H}_{2} \mathrm{SO}_{4}$ (general stain, after charring); $1 \%$ $N, N$-dimethyl-p-phenylenediamine solution in 1:20:100 AcOH$\mathrm{H}_{2} \mathrm{O}-\mathrm{MeOH}$ (specific for peroxides) ${ }^{16} 1 \%$ aq $\mathrm{KMnO}_{4}$ (for unsaturated compounds); $3 \%$ vanillin in $3 \% \mathrm{H}_{2} \mathrm{SO}_{4}$ in $\mathrm{EtOH}$ (general stain after charring). NMR spectra were recorded at $400 \mathrm{MHz}\left({ }^{1} \mathrm{H}\right)$ or 100 $\mathrm{MHz}\left({ }^{13} \mathrm{C}\right)$ and in $\mathrm{CDCl}_{3}$ unless otherwise indicated; peaks are reported as: chemical shift (multiplicity, $J$ couplings in $\mathrm{Hz}$, number of protons). IR spectra were recorded as neat films (ZnSe, ATR mode) with selected absorbances reported in wavenumbers $\left(\mathrm{cm}^{-1}\right)$. Melting points were collected using a melting point apparatus and all values are uncorrected unless otherwise noted.

\section{Hydroperoxyacetals from Alkenes; General Procedure 1 (GP 1)}

A solution of alkene $(\sim 1 \mathrm{mmol})$ and $\mathrm{MeOH}(0.3 \mathrm{~mL})$ in $\mathrm{CH}_{2} \mathrm{Cl}_{2}(10$ $\mathrm{mL}$ ) in a round-bottomed flask was cooled to $-78{ }^{\circ} \mathrm{C}$ and a gaseous stream of $2 \% \mathrm{O}_{3} / \mathrm{O}_{2}$ (approximately $1 \mathrm{mmol} \mathrm{O}_{3} / \mathrm{min}$ ) was bubbled through the solution. Once the pale blue color of ozone appeared, the ozonizer voltage was set to zero and the reaction was sparged with $\mathrm{O}_{2}$ until the blue color dissipated. The reaction was quenched with sat. aq $\mathrm{NaHCO}_{3}(15 \mathrm{~mL})$ and the resulting mixture was allowed to warm to r.t. The separated aqueous layer was extracted with $\mathrm{CH}_{2} \mathrm{Cl}_{2}(2 \times 25 \mathrm{~mL})$ and the combined organic layers were dried $\left(\mathrm{Na}_{2} \mathrm{SO}_{4}\right)$. The residue obtained upon concentration in vacuo was purified by flash chromatography using EtOAc-hexanes.
Carbonyl Compounds from Hydroperoxyacetals; General Procedure 2 (GP 2)

To a r.t. solution of hydroperoxyacetal $(\sim 0.5 \mathrm{mmol})$ in DCE (10 $\mathrm{mL})$ was added $\mathrm{NaBH}(\mathrm{OAc})_{3}(0.5 \mathrm{mmol})$. After product formation was complete (TLC, eluent: EtOAc-hexanes), the reaction was diluted with $\mathrm{CH}_{2} \mathrm{Cl}_{2}(\sim 40 \mathrm{~mL})$ and washed sequentially with sat. aq $\mathrm{NaHCO}_{3}(\sim 30 \mathrm{~mL})$ and brine $(\sim 30 \mathrm{~mL})$. The organic layer was dried $\left(\mathrm{Na}_{2} \mathrm{SO}_{4}\right)$ and the residue obtained upon concentration in vacuo was purified by flash chromatography using EtOAc-hexanes.

Amines from Hydroperoxyacetals; General Procedure 3 (GP 3) To a solution of hydroperoxyacetal $(\sim 0.5 \mathrm{mmol})$ in DCE $(10 \mathrm{~mL})$ was added $\mathrm{NaBH}(\mathrm{OAc})_{3}(0.5 \mathrm{mmol})$. The reaction mixture was stirred for an hour at r.t., after which additional $\mathrm{NaBH}(\mathrm{OAc})_{3}(1 \mathrm{mmol})$ was added followed by amine $(0.5 \mathrm{mmol})$. After the reaction appeared complete (TLC, eluent: EtOAc-hexanes), the solution was diluted with $\mathrm{CH}_{2} \mathrm{Cl}_{2}(40 \mathrm{~mL})$, and washed sequentially with sat. aq $\mathrm{NaHCO}_{3}$ $(30 \mathrm{~mL})$ and brine $(30 \mathrm{~mL})$. The organic layer was dried $\left(\mathrm{Na}_{2} \mathrm{SO}_{4}\right)$ and the residue obtained upon concentration was purified by flash chromatography with $\mathrm{CH}_{2} \mathrm{Cl}_{2}-\mathrm{MeOH}(1-2 \%)$ containing a few drops of $\mathrm{Et}_{3} \mathrm{~N}$.

\section{Direct Synthesis of Amines from Alkenes; General Procedure 4 (GP} 4)

Stepwise (Method A): A solution of alkene ( $1 \mathrm{mmol})$ and $\mathrm{MeOH}(0.3$ $\mathrm{mL})$ in $\mathrm{CH}_{2} \mathrm{Cl}_{2}(10 \mathrm{~mL})$ in a round-bottomed flask was ozonized as in GP 1. Following removal of free ozone, the cooling bath was removed and $\mathrm{NaBH}(\mathrm{OAc})_{3}(1 \mathrm{mmol})$ was added. The reaction mixture was stirred at r.t. for $45 \mathrm{~min}$, after which amine $(\sim 1 \mathrm{mmol})$ and $\mathrm{NaBH}(\mathrm{OAc})_{3}(2 \mathrm{mmol})$ were sequentially added. The mixture was stirred at r.t. for $1-2 \mathrm{~h}$ and then filtered through Celite and the pad washed with $\mathrm{CH}_{2} \mathrm{Cl}_{2}(20 \mathrm{~mL})$. The filtrate was concentrated on a rotary evaporator, and the residue was purified as in GP 3.

One Step (Method B): A solution of alkene ( 1 mmol) and $\mathrm{MeOH}(0.3$ $\mathrm{mL})$ in $\mathrm{CH}_{2} \mathrm{Cl}_{2}(10 \mathrm{~mL})$ in a round-bottomed flask was ozonized as in GP 1. Following removal of free ozone, the cooling bath was removed and $\mathrm{NaBH}(\mathrm{OAc})_{3}(3 \mathrm{mmol})$ and the amine $(1-2 \mathrm{mmol})$ were sequentially added. The reaction mixture was stirred at r.t. for $1-2 \mathrm{~h}$ and then filtered through Celite and the pad washed with $\mathrm{CH}_{2} \mathrm{Cl}_{2}$ $(20 \mathrm{~mL})$. The filtrate was concentrated on a rotary evaporator, and the residue was purified as in GP 3 .

Hydroperoxyacetals from Alkenes1-Methoxynonyl Hydroperoxide (1b) [20525-41-1]

Prepared from $1 \mathrm{a}(1.40 \mathrm{~g}, 10 \mathrm{mmol})$ by GP 1 ; yield: $1.33 \mathrm{~g}(70 \%) ; R_{f}=$ 0.22 (10\% EtOAc-hexanes).

${ }^{1} \mathrm{H}$ NMR: $\delta=8.19(\mathrm{~s}, 1 \mathrm{H}), 4.76(\mathrm{t}, J=5.6 \mathrm{~Hz}, 1 \mathrm{H}), 3.52(\mathrm{~s}, 3 \mathrm{H}), 1.60-$ $1.80(\mathrm{~m}, 2 \mathrm{H}), 1.20-1.50(\mathrm{~m}, 12 \mathrm{H}), 0.90(\mathrm{t}, J=6.8 \mathrm{~Hz}, 3 \mathrm{H})$.

${ }^{13} \mathrm{C}$ NMR: $\delta=108.8,55.8,31.9,31.3,29.43,29.38,29.3,24.6,22.7,14.1$.

9-Hydroperoxy-9-methoxynonanol Acetate Ester (2b) [1255126-65-8] Prepared from $2 a(2.0 \mathrm{~g}, 10 \mathrm{mmol})$ by GP 1 ; yield: $1.66 \mathrm{~g}(67 \%) ; R_{f}=$ 0.33 (25\% EtOAc-hexanes).

${ }^{1} \mathrm{H}$ NMR (300 MHz): $\delta=9.46(\mathrm{~s}, 1 \mathrm{H}), 4.66(\mathrm{t}, J=5.8 \mathrm{~Hz}, 1 \mathrm{H}), 3.97(\mathrm{t}$, $J=6.7 \mathrm{~Hz}, 2 \mathrm{H}), 3.43(\mathrm{~s}, 3 \mathrm{H}), 1.98(\mathrm{~s}, 3 \mathrm{H}), 1.66-1.48(\mathrm{~m}, 4 \mathrm{H}), 1.33-$ $1.23(\mathrm{~m}, 10 \mathrm{H})$.

${ }^{13} \mathrm{C}$ NMR $(75 \mathrm{MHz}): \delta=171.6,108.5,64.7,55.7,31.3,29.2,29.1,28.9$, 28.4, 25.7, 24.5, 20.9.

4-Benzyloxy-1-methoxybutyl Hydroperoxide (3b) [1255126-67-0] Prepared from $3 a(1.76 \mathrm{~g}, 10 \mathrm{mmol})$ by GP 1 ; yield: $1.56 \mathrm{~g}(69 \%) ; R_{f}=$ 0.37 (25\% EtOAc-hexanes). 
${ }^{1} \mathrm{H}$ NMR: $\delta=8.93(\mathrm{~s}, 1 \mathrm{H}), 7.39-7.29(\mathrm{~m}, 5 \mathrm{H}), 4.77-4.74(\mathrm{~m}, 1 \mathrm{H}), 4.54$ (s, $2 \mathrm{H}), 3.58-3.50(\mathrm{~s}, 5 \mathrm{H}), 1.93-1.71(\mathrm{~m}, 4 \mathrm{H})$.

${ }^{13} \mathrm{C}$ NMR: $\delta=138.1,128.4,127.8,127.7,108.4,72.9,69.8,55.9,28.0$, 24.6.

\section{(3-Hydroperoxy-3-methoxybutyl)benzene (4b)}

Prepared from $4 a(1.0 \mathrm{~g}, 5.6 \mathrm{mmol})$ by GP 1; yield: $0.427 \mathrm{~g}(38 \%) ; R_{f}=$ 0.36 (25\% EtOAc-hexanes).

IR (neat): 3345, 2943, 1102, 1069, $697 \mathrm{~cm}^{-1}$.

${ }^{1} \mathrm{H}$ NMR: $\delta=7.49$ (s, $\left.1 \mathrm{H}\right), 7.31-7.22(\mathrm{~m}, 5 \mathrm{H}), 3.37$ (s, $\left.3 \mathrm{H}\right), 2.76-2.69$ $(\mathrm{m}, 2 \mathrm{H}), 2.08-2.02(\mathrm{~m}, 2 \mathrm{H}), 1.45(\mathrm{~s}, 3 \mathrm{H})$.

${ }^{13}$ C NMR: $\delta=141.7,128.5,128.3,126.0,106.9,49.1,36.9,30.5,19.1$.

HRMS-ESI: $m / z$ calcd for $\mathrm{C}_{11} \mathrm{H}_{16} \mathrm{O}_{3}+\mathrm{Na}(\mathrm{M}+\mathrm{Na})^{+}:$219.0997; found: 219.1007.

\section{4-(t-Butyl)-1-hydroperoxy-1-methoxycyclohexane} [169294-55-7]

Prepared as a mixture of diastereomers from $5 a(1.52 \mathrm{~g}, 10 \mathrm{mmol})$ by GP 1; yield: $1.49 \mathrm{~g}$ (74\%); $R_{f}=0.41$ (20\% EtOAc-hexanes).

${ }^{1} \mathrm{H}$ NMR: $\delta=7.50(\mathrm{~s}, 0.25 \mathrm{H}), 7.48(\mathrm{~s}, 0.68 \mathrm{H}), 3.34(\mathrm{~s}, 0.79 \mathrm{H}), 3.31$ (s, $2.18 \mathrm{H}), 2.20-2.28(\mathrm{~m}, 0.54 \mathrm{H}), 2.09-2.19(\mathrm{~m}, 1.52 \mathrm{H}), 1.66-177(\mathrm{~m}, 2 \mathrm{H})$, 1.33-1.46 (m, 2 H), 1.12-1.32 (m, 2 H), 1.00-1.11 (m, 1 H), 0.89 (s, 9 H). ${ }^{13} \mathrm{C}$ NMR: $\delta=105.7,105.4,48.5,48.3,47.6,47.5,32.3,31.5,30.9,27.6$, 23.6, 23.4 .

2-Hydroperoxy-2-methoxyadamantane (6b) [55975-21-8]

Prepared from $6 a(1.0 \mathrm{~g}, 5.61 \mathrm{mmol})$ by GP 1 ; yield: $0.700 \mathrm{~g}(63 \%) ; R_{f}$ $=0.50$ (25\% EtOAc-hexanes).

${ }^{1} \mathrm{H}$ NMR: $\delta=7.34$ (s, $\left.1 \mathrm{H}\right), 3.32$ (s, $\left.3 \mathrm{H}\right), 2.23$ (m, $\left.2 \mathrm{H}\right), 2.01-1.86(\mathrm{~m}, 6$ $\mathrm{H}), 1.70-1.66(\mathrm{~m}, 6 \mathrm{H})$.

${ }^{13}$ C NMR: $\delta=107.5,47.4,37.1,33.71,33.68,31.9,27.0,26.9$.

\section{Carbonyls from HydroperoxyacetalsNonanal (1c) [124-19-6]}

Prepared from $1 b(0.095 \mathrm{~g}$, $0.5 \mathrm{mmol})$ by GP 2; reaction time: $2 \mathrm{~h}$; yield: $0.045 \mathrm{~g}(63 \%) ; R_{f}=0.66$ (10\% EtOAc-hexanes).

${ }^{1} \mathrm{H}$ NMR: $\delta=9.78(\mathrm{t}, J=1.8 \mathrm{~Hz}, 1 \mathrm{H}), 2.43(\mathrm{dt}, J=1.8,7.3 \mathrm{~Hz}, 2 \mathrm{H})$, $1.64(\mathrm{~m}, 2 \mathrm{H}), 1.31-1.28(\mathrm{~m}, 10 \mathrm{H}), 0.89(\mathrm{t}, J=6.9 \mathrm{~Hz}, 3 \mathrm{H})$.

${ }^{13} \mathrm{C}$ NMR: $\delta=203.0,43.9,31.8,29.3,29.2,29.1,22.6,22.0,14.0$.

\section{9-Hydroxynonanal Acetate Ester (2c) [29541-97-7]}

Prepared from $2 b$ (0.496 g, $2 \mathrm{mmol})$ by GP 2; reaction time: $2 \mathrm{~h}$; yield: $0.341 \mathrm{~g}(85 \%) ; R_{f}=0.44$ (25\% EtOAc-hexanes).

${ }^{1} \mathrm{H}$ NMR: $\delta=9.78(\mathrm{t}, J=1.8 \mathrm{~Hz}, 1 \mathrm{H}), 4.07(\mathrm{t}, J=6.8 \mathrm{~Hz}, 2 \mathrm{H}), 2.45(\mathrm{dt}$, $J=1.8,7.3 \mathrm{~Hz}, 2 \mathrm{H}), 2.07(\mathrm{~s}, 3 \mathrm{H}), 1.64(\mathrm{~m}, 4 \mathrm{H}), 1.34(\mathrm{~m}, 8 \mathrm{H})$.

${ }^{13} \mathrm{C}$ NMR: $\delta=202.7,171.1,64.5,43.8,29.1,28.97,28.95,28.5,25.8$, $21.9,20.9$.

\section{4-(Benzyloxy)butanal (3c) [5470-84-8]}

Prepared from $3 b(0.107 \mathrm{~g}, 0.47 \mathrm{mmol})$ by GP 2; reaction time: $2 \mathrm{~h}$; yield: $0.076 \mathrm{~g}(91 \%) ; R_{f}=0.33$ (25\% EtOAc-hexanes).

${ }^{1} \mathrm{H}$ NMR: $\delta=9.79(\mathrm{t}, J=1.5 \mathrm{~Hz}, 1 \mathrm{H}), 7.39-7.27(\mathrm{~m}, 5 \mathrm{H}), 4.51(\mathrm{~s}, 2 \mathrm{H})$, $3.53(\mathrm{t}, J=6.0 \mathrm{~Hz}, 2 \mathrm{H}), 2.58(\mathrm{dt}, J=1.32,7.1 \mathrm{~Hz}, 2 \mathrm{H}$ ), 1.98 (quint, $J=$ $6.5 \mathrm{~Hz}, 2 \mathrm{H})$.

${ }^{13}$ C NMR: $\delta=202.3,138.3,128.4,127.6,72.9,69.1,40.9,22.6$.

\section{4-Phenylbutan-2-one (4c) [2550-26-7]}

Prepared from $4 b(0.098 \mathrm{~g}, 0.5 \mathrm{mmol})$ by GP 2; reaction time: $5 \mathrm{~h}$; yield: $0.052 \mathrm{~g}(70 \%) ; R_{f}=0.37$ (25\% EtOAc-hexanes).

${ }^{1} \mathrm{H}$ NMR: $\delta=7.33-7.29$ (m, $\left.2 \mathrm{H}\right)$, 7.24-7.20 (m, $\left.3 \mathrm{H}\right), 2.92(\mathrm{t}, J=7.6 \mathrm{~Hz}$,
$2 \mathrm{H}), 2.79(\mathrm{t}, J=7.6 \mathrm{~Hz}, 2 \mathrm{H}), 2.16(\mathrm{~s}, 3 \mathrm{H})$.

${ }^{13}$ C NMR: $\delta=207.9,141.0,128.5,128.3,126.1,45.2,30.1,29.7$.

4-(tert-Butyl)cyclohexanone (5c) [98-53-3]

Prepared from $5 b(0.101 \mathrm{~g}, 0.5 \mathrm{mmol})$ by GP 2; reaction time: $5 \mathrm{~h}$; yield: $0.053 \mathrm{~g}(68 \%) ; R_{f}=0.50$ (25\% EtOAc-hexanes).

${ }^{1} \mathrm{H}$ NMR: $\delta=2.40-2.26(\mathrm{~m}, 4 \mathrm{H}), 2.09-2.06(\mathrm{~m}, 2 \mathrm{H}), 1.52-1.38(\mathrm{~m}, 3$ $\mathrm{H}), 0.91$ (s, $9 \mathrm{H})$.

${ }^{13} \mathrm{C}$ NMR: $\delta=212.5,46.7,41.3,32.4,27.6$.

Adamantan-2-one (6c) [700-58-3]

Prepared from $6 b(0.099 \mathrm{~g}$, $0.5 \mathrm{mmol})$ by GP 2; reaction time: $8 \mathrm{~h}$; yield: $0.044 \mathrm{~g}$ [ $68 \%$ based on $0.015 \mathrm{~g}(15 \%)$ starting material recovery]; $R_{f}=0.47$ ( $25 \%$ EtOAc-hexanes).

${ }^{1} \mathrm{H}$ NMR: $\delta=2.54(\mathrm{~m}, 2 \mathrm{H}), 2.09-1.92(\mathrm{~m}, 12 \mathrm{H})$.

${ }^{13} \mathrm{C}$ NMR: $\delta=218.4,46.9,39.2,36.3,27.4$.

Amines from Hydroperoxyacetals9-Morpholinononanol Acetate Ester $(2 d)$

Prepared from $2 b(0.124 \mathrm{~g}, 0.5 \mathrm{mmol})$ with morpholine $(0.5 \mathrm{mmol})$ by GP 3; reaction time: $5 \mathrm{~h}$; yield: $0.115 \mathrm{~g}(85 \%) ; R_{f}=0.32(10 \%$ $\mathrm{MeOH}-\mathrm{CH}_{2} \mathrm{Cl}_{2}$ ).

IR (neat): 2927, 2853, 1738, $1118 \mathrm{~cm}^{-1}$.

${ }^{1} \mathrm{H}$ NMR: $\delta=4.05(\mathrm{t}, J=6.8 \mathrm{~Hz}, 2 \mathrm{H}), 3.72(\mathrm{t}, J=4.4 \mathrm{~Hz}, 4 \mathrm{H}), 2.44(\mathrm{~m}$, $4 \mathrm{H}), 2.32(\mathrm{t}, J=7.4 \mathrm{~Hz}, 2 \mathrm{H}), 2.04(\mathrm{~s}, 3 \mathrm{H}), 1.65-1.57(\mathrm{~m}, 2 \mathrm{H}), 1.48(\mathrm{~m}$, $2 \mathrm{H}), 1.35-1.29(\mathrm{~m}, 10 \mathrm{H})$.

${ }^{13} \mathrm{C}$ NMR: $\delta=171.2,66.9,64.6,59.2,53.8,29.42,29.38,29.1,28.5,27.4$, 26.5, 25.8, 20.9 .

HRMS-FAB: $m / z$ calcd for $\mathrm{C}_{15} \mathrm{H}_{30} \mathrm{NO}_{3}(\mathrm{M}+\mathrm{H})^{+}$: 272.2226; found: 272.2213 .

N-Phenyl-9-aminononanol Acetate Ester (2e)

Prepared from $2 b(0.124 \mathrm{~g}, 0.5 \mathrm{mmol})$ with aniline $(0.5 \mathrm{mmol})$ by GP 3; reaction time: $5 \mathrm{~h}$; yield: $0.104 \mathrm{~g}(75 \%) ; R_{f}=0.50(10 \%$ EtOAc-hexanes).

IR (neat): 3401, 2926, 2854, 1734, $1236 \mathrm{~cm}^{-1}$.

${ }^{1} \mathrm{H}$ NMR: $\delta=7.22-7.18(\mathrm{~m}, 2 \mathrm{H}), 6.74-6.62(\mathrm{~m}, 3 \mathrm{H}), 4.09(\mathrm{t}, J=6.76$ $\mathrm{Hz}, 2 \mathrm{H}), 3.66(\mathrm{~s}, 1 \mathrm{H}), 3.13(\mathrm{t}, J=7.16 \mathrm{~Hz}, 2 \mathrm{H}), 2.08(\mathrm{~s}, 3 \mathrm{H}), 1.68-1.63$ $(\mathrm{m}, 4 \mathrm{H}), 1.43-1.36(\mathrm{~m}, 10 \mathrm{H})$.

${ }^{13} \mathrm{C}$ NMR: $\delta=171.3,148.6,129.2,117.0,112.7,64.6,43.9,29.6,29.5$, 29.4, 29.2, 28.6, 27.2, 25.9, 21.1.

HRMS-FAB: $m / z$ calcd for $\mathrm{C}_{17} \mathrm{H}_{28} \mathrm{NO}_{2}(\mathrm{M}+\mathrm{H})^{+}:$278.2115; found: 278.2123.

N-Benzyl-9-aminononanol Acetate Ester (2f)

Prepared from $2 b(0.124 \mathrm{~g}, 0.5 \mathrm{mmol})$ with benzylamine $(2.5 \mathrm{mmol})$ by GP 3; reaction time: $5 \mathrm{~h}$; yield: $0.098 \mathrm{~g}(67 \%) ; R_{f}=0.42(10 \%$ $\mathrm{MeOH}-\mathrm{CH}_{2} \mathrm{Cl}_{2}$ ).

IR (neat): 2923, 2856, 1737, $1236 \mathrm{~cm}^{-1}$.

${ }^{1} \mathrm{H}$ NMR: $\delta=7.39-7.25(\mathrm{~m}, 5 \mathrm{H}), 4.21(\mathrm{~s}, 1 \mathrm{H}), 4.04(\mathrm{t}, J=6.8 \mathrm{~Hz}, 2 \mathrm{H})$ $3.84(\mathrm{~s}, 2 \mathrm{H}), 2.65(\mathrm{t}, J=7.5 \mathrm{~Hz}, 2 \mathrm{H}), 2.04(\mathrm{~s}, 3 \mathrm{H}), 1.62-1.59(\mathrm{~m}, 4 \mathrm{H})$, $1.28(\mathrm{~m}, 10 \mathrm{H})$.

${ }^{13} \mathrm{C}$ NMR: $\delta=171.2,138.2,128.6,128.5,127.4,64.6,53.3,48.7,29.4$, 29.3, 29.1, 29.1, 28.6, 27.2, 25.9, 21.0.

HRMS-ESI: $m / z$ calcd for $\mathrm{C}_{15} \mathrm{H}_{29} \mathrm{NO}_{2}+\mathrm{Na}(\mathrm{M}+\mathrm{Na})^{+}: 314.2091$; found: 314.2110 . 


\section{$N, N^{\prime}-(B i s-9-a c e t o x y n o n y l) b e n z y l a m i n e$}

The tertiary amine, $N, N^{\prime}$-(bis-9-acetoxynonyl)benzylamine, resulting from the reductive amination of $2 f$ by $2 b$ was also isolated in $8 \%$ yield; $R_{f}=0.65\left(10 \% \mathrm{MeOH}-\mathrm{CH}_{2} \mathrm{Cl}_{2}\right)$.

IR (neat): 2925, 2855, 1738, 1232, $1043 \mathrm{~cm}^{-1}$.

${ }^{1} \mathrm{H}$ NMR: $\delta=7.35-7.22(\mathrm{~m}, 5 \mathrm{H}), 4.06(\mathrm{t}, J=6.8 \mathrm{~Hz}, 4 \mathrm{H}), 3.56(\mathrm{~s}, 2 \mathrm{H})$, $2.40(\mathrm{t}, J=7.2 \mathrm{~Hz}, 4 \mathrm{H}), 2.06(\mathrm{~s}, 6 \mathrm{H}), 1.66-1.59(\mathrm{~m}, 4 \mathrm{H}), 1.47-1.45(\mathrm{~m}$, $4 \mathrm{H}), 1.34-1.27(20 \mathrm{H})$.

${ }^{13} \mathrm{C}$ NMR: $\delta=171.2,140.5,128.8,128.0,126.6,64.6,58.6,53.8,29.5$, $29.4,29.2,28.6,27.4,27.0,25.9,21.0$.

HRMS-FAB Calcd for $\mathrm{C}_{29} \mathrm{H}_{50} \mathrm{NO}_{4}(\mathrm{M}+\mathrm{H})^{+}$: 476.3740; found: 476.3725 .

\section{N-Phenethyl-9-aminononanol Acetate Ester (2g)}

Prepared from $2 b(0.124 \mathrm{~g}, 0.5 \mathrm{mmol})$ with phenethylamine $(2 \mathrm{mmol})$ by GP 3; reaction time: $5 \mathrm{~h}$; yield: $0.096 \mathrm{~g}(63 \%) ; R_{f}=0.60(10 \%$ $\mathrm{MeOH}-\mathrm{CH}_{2} \mathrm{Cl}_{2}$ ).

IR (neat): 3676, 2988, 2901, $1736 \mathrm{~cm}^{-1}$.

${ }^{1} \mathrm{H}$ NMR: $\delta=7.32-7.19(\mathrm{~m}, 5 \mathrm{H}), 4.06(\mathrm{t}, J=6.7 \mathrm{~Hz}, 2 \mathrm{H}), 2.91-2.87(\mathrm{~m}$, $2 \mathrm{H}), 2.84-2.80(\mathrm{~m}, 2 \mathrm{H}), 2.62(\mathrm{t}, J=7.3 \mathrm{~Hz}, 2 \mathrm{H}), 2.06(\mathrm{~s}, 3 \mathrm{H}), 1.62(\mathrm{q}, J$ $=7.2 \mathrm{~Hz}, 2 \mathrm{H}), 1.47(\mathrm{~m}, 2 \mathrm{H}), 1.29(\mathrm{~m}, 10 \mathrm{H})$.

${ }^{13} \mathrm{C}$ NMR: $\delta=171.3,140.2,128.7,128.4,126.1,64.6,51.3,49.9,36.5$, 30.1, 29.5, 29.4, 29.2, 28.6, 27.3, 25.9, 21.0.

HRMS-FAB: $m / z$ calcd for $\mathrm{C}_{19} \mathrm{H}_{32} \mathrm{NO}_{2}(\mathrm{M}+\mathrm{H})^{+}$: 306.2428; found: 306.2439.

\section{4-[4-(Benzyloxy)butyl]morpholine (3d)}

Prepared from $3 b(0.107 \mathrm{~g}, 0.47 \mathrm{mmol})$ with morpholine $(0.5 \mathrm{mmol})$ by GP 3; reaction time: $5 \mathrm{~h}$; yield: $0.104 \mathrm{~g}(89 \%) ; R_{f}=0.33(5 \%$ $\mathrm{MeOH}-\mathrm{CH}_{2} \mathrm{Cl}_{2}$ ).

IR (neat): 2942, 2853, 2806, $1116 \mathrm{~cm}^{-1}$.

${ }^{1} \mathrm{H}$ NMR: $\delta=7.34-7.27(\mathrm{~m}, 5 \mathrm{H}), 4.50(\mathrm{~s}, 2 \mathrm{H}), 3.72(\mathrm{t}, J=4.7 \mathrm{~Hz}, 4 \mathrm{H})$, $3.49(\mathrm{t}, J=6.1 \mathrm{~Hz}, 2 \mathrm{H}), 2.44(\mathrm{~m}, 4 \mathrm{H}), 2.35(\mathrm{t}, J=7.2 \mathrm{~Hz}, 2 \mathrm{H}), 1.66-$ $1.56(\mathrm{~m}, 4 \mathrm{H})$.

${ }^{13} \mathrm{C}$ NMR: $\delta=138.6,128.4,127.6,127.5,72.9,70.1,66.9,58.8,53.7$, 27.7, 23.2.

HRMS-FAB: $m / z$ calcd for $\mathrm{C}_{15} \mathrm{H}_{24} \mathrm{NO}_{2}(\mathrm{M}+\mathrm{H})^{+}$: 250.1802; found: 250.1812 .

\section{N-[4-(Benzyloxy)butyl]aniline (3e)}

Prepared from $3 b(0.107 \mathrm{~g}, 0.47 \mathrm{mmol})$ with aniline $(0.5 \mathrm{mmol})$ by GP 3; reaction time: $4 \mathrm{~h}$; yield: $0.083 \mathrm{~g}(69 \%) ; R_{f}=0.51(20 \%$ EtOAc-hexanes).

IR (neat): 3403, 3026, 2935, 2858, 1601, $1505 \mathrm{~cm}^{-1}$.

${ }^{1} \mathrm{H}$ NMR: $\delta=7.38-7.30(\mathrm{~m}, 5 \mathrm{H}), 7.21-7.17(\mathrm{~m}, 2 \mathrm{H}), 6.71(\mathrm{t}, J=7.3 \mathrm{~Hz}$, $1 \mathrm{H}), 6.61-6.59(\mathrm{~m}, 2 \mathrm{H}), 4.54(\mathrm{~s}, 2 \mathrm{H}), 3.73(\mathrm{~s}, 1 \mathrm{H}), 3.55(\mathrm{t}, J=5.9 \mathrm{~Hz}, 2$ $\mathrm{H}), 3.18-3.15(\mathrm{~m}, 2 \mathrm{H}), 1.79-1.57(\mathrm{~m}, 4 \mathrm{H})$.

${ }^{13}$ C NMR: $\delta=148.6,138.6,129.3,128.5,127.8,127.7,117.2,112.8,73.1$, $70.2,43.8,27.5,26.5$

HRMS-FAB: $m / z$ calcd for $\mathrm{C}_{17} \mathrm{H}_{22} \mathrm{NO}(\mathrm{M}+\mathrm{H})^{+}$: 256.1696; found: 256.1692.

N-[4-(Benzyloxy)butyl]benzylamine (3f) [60058-23-3]

Prepared from $3 b(0.107 \mathrm{~g}$, $0.47 \mathrm{mmol})$ with benzylamine $(2.5 \mathrm{mmol})$ by GP 3; reaction time: $5 \mathrm{~h}$; yield: $0.079 \mathrm{~g}(63 \%) ; R_{f}=0.51(10 \%$ $\mathrm{MeOH}-\mathrm{CH}_{2} \mathrm{Cl}_{2}$ )

${ }^{1} \mathrm{H}$ NMR: $\delta=7.39-7.24(\mathrm{~m}, 10 \mathrm{H}), 4.52(\mathrm{~s}, 2 \mathrm{H}), 3.80(\mathrm{~s}, 2 \mathrm{H}), 3.50(\mathrm{t}, J=$ $6.2 \mathrm{~Hz}, 2 \mathrm{H}), 2.67(\mathrm{t}, J=6.9 \mathrm{~Hz}, 2 \mathrm{H}), 1.73-1.59(\mathrm{~m}, 4 \mathrm{H}), 1.55(\mathrm{~s}, 1 \mathrm{H})$.

${ }^{13}$ C NMR: $\delta=140.5,138.6,128.4,128.3,128.1,127.6,127.5,126.9,72.9$, $70.3,54.0,49.2,27.6,26.8$.
N-[4-(Benzyloxy)butyl)]-2-phenethylamine (3g)

Prepared from $3 b(0.107 \mathrm{~g}, 0.47 \mathrm{mmol})$ with phenethylamine $(2.5$ $\mathrm{mmol})$ by GP 3; reaction time: $4 \mathrm{~h}$; yield: $0.087 \mathrm{~g}(65 \%) ; R_{f}=0.22(5 \%$ $\mathrm{MeOH}-\mathrm{CH}_{2} \mathrm{Cl}_{2}$ ).

IR (neat): 3026, 2932, 2854, 1097, $696 \mathrm{~cm}^{-1}$.

${ }^{1} \mathrm{H}$ NMR: $\delta=7.37-7.22(\mathrm{~m}, 10 \mathrm{H}), 4.52(\mathrm{~s}, 2 \mathrm{H}), 3.50(\mathrm{t}, J=6.2 \mathrm{~Hz}, 2$ $\mathrm{H}), 2.92-2.88(\mathrm{~m}, 2 \mathrm{H}), 2.84-2.80(\mathrm{~m}, 2 \mathrm{H}), 2.68-2.65(\mathrm{t}, J=7.1 \mathrm{~Hz}, 2 \mathrm{H})$, 1.69-1.55 (m, $4 \mathrm{H}), 1.37(\mathrm{~s}, 1 \mathrm{H})$.

${ }^{13}$ C NMR: $\delta=140.1,138.6,128.7,128.5,128.4,127.6,127.5,126.1,72.9$, 70.3, 51.2, 49.7, 36.5, 27.6, 26.9.

HRMS-FAB: $m / z$ calcd for $\mathrm{C}_{19} \mathrm{H}_{26} \mathrm{NO}(\mathrm{M}+\mathrm{H})^{+}$: 284.2009; found: 284.2018.

4-(4-Phenylbutan-2-yl)morpholine (4d) [2832-95-3]

Prepared from $4 b(0.098 \mathrm{~g}$, $0.5 \mathrm{mmol})$ with morpholine $(0.5 \mathrm{mmol})$ by GP 3; reaction time: $24 \mathrm{~h}$; yield: $0.049 \mathrm{~g}(45 \%) ; R_{f}=0.37(5 \%$ $\mathrm{MeOH}-\mathrm{CH}_{2} \mathrm{Cl}_{2}$ ).

${ }^{1} \mathrm{H}$ NMR: $\delta=7.32-7.18(\mathrm{~m}, 5 \mathrm{H}), 3.75-3.72(\mathrm{~m}, 4 \mathrm{H}), 2.75-2.62(\mathrm{~m}, 2$ $\mathrm{H}), 2.60-2.52-2.44(\mathrm{~m}, 5 \mathrm{H}), 1.90-1.83(\mathrm{~m}, 1 \mathrm{H}), 1.66(\mathrm{~m}, 1 \mathrm{H}), 1.64-1.54$ $(\mathrm{m}, 1 \mathrm{H}), 1.03(\mathrm{~d}, J=6.7 \mathrm{~Hz}, 2 \mathrm{H})$.

${ }^{13} \mathrm{C}$ NMR: $\delta=142.6,128.4,128.3,125.7,67.5,58.4,48.7,35.3,32.8$, 13.9 .

N-(4-Phenylbutan-2-yl)aniline (4e) [72641-00-0]

Prepared from $4 b(0.098 \mathrm{~g}, 0.5 \mathrm{mmol})$ with aniline $(0.5 \mathrm{mmol})$ by GP 3; reaction time: $36 \mathrm{~h}$; yield: $0.068 \mathrm{~g}(60 \%) ; R_{f}=0.51(10 \%$ EtOAc-hexanes).

${ }^{1} \mathrm{H}$ NMR: $\delta=7.33-7.28(\mathrm{~m}, 2 \mathrm{H}), 7.23-7.15(\mathrm{~m}, 5 \mathrm{H}), 6.69(\mathrm{t}, J=7.3 \mathrm{~Hz}$, $1 \mathrm{H}), 6.56(\mathrm{~d}, J=8.2 \mathrm{~Hz}, 2 \mathrm{H}), 3.52(\mathrm{~m}, 1 \mathrm{H}), 3.45(\mathrm{~s}, 1 \mathrm{H}), 2.75(\mathrm{t}, J=$ $7.8 \mathrm{~Hz}, 2 \mathrm{H}), 1.95-1.86(\mathrm{~m}, 1 \mathrm{H}), 1.84-1.75(\mathrm{~m}, 1 \mathrm{H}), 1.24(\mathrm{~d}, J=6.3 \mathrm{~Hz}$, $3 \mathrm{H})$.

${ }^{13}$ C NMR: $\delta=147.6,142.0,129.3,128.5,128.4,125.9,116.9,113.2,47.9$, $38.8,32.5,20.9$

\section{N-Benzyl-4-phenylbutan-2-amine (4f) [68164-04-5]}

Prepared from $4 b(0.098 \mathrm{~g}, 0.5 \mathrm{mmol})$ with benzylamine $(0.5 \mathrm{mmol})$ by GP 3; reaction time: $36 \mathrm{~h}$; yield: $0.089 \mathrm{~g}(75 \%) ; R_{f}=0.25(5 \%$ $\mathrm{MeOH}-\mathrm{CH}_{2} \mathrm{Cl}_{2}$ ).

${ }^{1} \mathrm{H}$ NMR: $\delta=7.36-7.20(\mathrm{~m}, 10 \mathrm{H}), 3.87(\mathrm{~d}, J=13.0 \mathrm{~Hz}, 1 \mathrm{H}), 3.77(\mathrm{~d}, J$ $=13.0 \mathrm{~Hz}, 1 \mathrm{H}), 2.82-2.64(\mathrm{~m}, 3 \mathrm{H}), 1.90-1.81(\mathrm{~m}, 1 \mathrm{H}), 1.76-1.66(\mathrm{~m}, 1$ $\mathrm{H}), 1.41(\mathrm{~s}, 1 \mathrm{H}), 1.19(\mathrm{~d}, J=6.4 \mathrm{~Hz}, 3 \mathrm{H})$.

${ }^{13} \mathrm{C}$ NMR: $\delta=142.5,140.7,128.44,128.39,128.2,127.9,126.9,125.7$, $52.0,51.3,38.7,32.3,20.4$.

N-Phenethyl-4-phenylbutan-2-amine (4g) [161012-69-7]

Prepared from $4 b(0.138 \mathrm{~g}, 0.7 \mathrm{mmol})$ with phenethylamine by GP 3; reaction time: $30 \mathrm{~h}$; yield: $0.092 \mathrm{~g}(52 \%) ; R_{f}=0.30(10 \%$ $\mathrm{MeOH}-\mathrm{CH}_{2} \mathrm{Cl}_{2}$ ).

${ }^{1} \mathrm{H}$ NMR: $\delta$ = 7.36-7.15 (m, $\left.10 \mathrm{H}\right), 2.99-2.91(\mathrm{~m}, 1 \mathrm{H}), 2.88-2.79(\mathrm{~m}, 3$ $\mathrm{H}), 2.72-2.66(\mathrm{~m}, 1 \mathrm{H}), 2.64-2.57(\mathrm{~m}, 2 \mathrm{H}), 1.84-1.75(\mathrm{~m}, 1 \mathrm{H}), 1.68-1.59$ $(\mathrm{m}, 1 \mathrm{H}), 1.30(\mathrm{~s}, 1 \mathrm{H}), 1.13(\mathrm{~d}, J=6.3 \mathrm{~Hz}, 3 \mathrm{H})$.

${ }^{13}$ C NMR: $\delta=142.3,140.1,128.7,128.5,128.4,128.3,126.2,125.7,55.4$, $48.5,38.7,36.6,32.3,20.3$.

Amines from Alkenes 1-Benzylazapine (7) [20422-13-3]

Prepared by reacting cyclohexene $(0.082 \mathrm{~g}, 1 \mathrm{mmol})$ with benzylamine (1 mmol) according to GP 4A; reaction time: $2 \mathrm{~h}$; yield: $0.107 \mathrm{~g}$ $(57 \%) ; R_{f}=0.57\left(10 \% \mathrm{MeOH}-\mathrm{CH}_{2} \mathrm{Cl}_{2}\right)$.

${ }^{1} \mathrm{H}$ NMR: $\delta=7.40-7.27$ (m, 5 H), $3.69(\mathrm{~s}, 2 \mathrm{H}), 2.67-2.66(\mathrm{~m}, 4 \mathrm{H}), 1.67$ $(\mathrm{m}, 8 \mathrm{H})$.

${ }^{13}$ C NMR: $\delta=140.1,128.8,128.1,126.7,62.7,55.6,28.2,27.0$. 
1-Phenethylazapine (8) [65530-43-0]

Prepared by reacting cyclohexene $(0.082 \mathrm{~g}, 1 \mathrm{mmol})$ with phenethylamine ( $1 \mathrm{mmol}$ ) according to GP 4A; reaction time: 2 h; yield: $0.127 \mathrm{~g}$ $(63 \%) ; R_{f}=0.57\left(10 \% \mathrm{MeOH}-\mathrm{CH}_{2} \mathrm{Cl}_{2}\right)$.

${ }^{1} \mathrm{H}$ NMR: $\delta=7.32-7.21(\mathrm{~m}, 5 \mathrm{H}), 2.83-2.80(\mathrm{~m}, 8 \mathrm{H}), 1.72(\mathrm{~m}, 4 \mathrm{H}), 1.64$ $(\mathrm{m}, 4 \mathrm{H})$.

${ }^{13}$ C NMR: $\delta=140.2,128.8,128.4,126.0,59.9,55.1,33.6,27.2,27.1$.

\section{4-[4-(Benzyloxy)butyl]morpholine (3d)}

Prepared from $3 a(0.176 \mathrm{~g}, 1.0 \mathrm{mmol})$ with morpholine $(1 \mathrm{mmol})$ by GP 4A; reaction time: $2 \mathrm{~h}$; yield: $0.159 \mathrm{~g}(64 \%)$. Spectral data were identical with those of compound $3 d$ described above.

\section{1-Benzylpiperidine (9) [2905-56-8]}

Prepared by reacting cyclopentene $(0.068 \mathrm{~g}, 1 \mathrm{mmol})$ with benzylamine ( $1 \mathrm{mmol}$ ) according to GP 4A; reaction time: $2 \mathrm{~h}$; yield: $0.113 \mathrm{~g}$ $(65 \%) ; R_{f}=0.50\left(10 \% \mathrm{MeOH}-\mathrm{CH}_{2} \mathrm{Cl}_{2}\right)$.

${ }^{1} \mathrm{H}$ NMR: $\delta=7.35-7.24(\mathrm{~m}, 5 \mathrm{H}), 3.50(\mathrm{~s}, 2 \mathrm{H}), 2.40-2.39(\mathrm{~m}, 4 \mathrm{H}), 1.64-$ $1.57(\mathrm{~m}, 4 \mathrm{H}), 1.49-1.46(\mathrm{~m}, 2 \mathrm{H})$.

${ }^{13} \mathrm{C}$ NMR: $\delta=138.7,129.2,128.0,126.8,63.9,54.5,26.0,24.4$.

\section{1-Phenethylpiperidine (10) [332-14-9]}

Prepared by reacting cyclopentene $(0.068 \mathrm{~g}, 1 \mathrm{mmol})$ with phenethylamine ( $1 \mathrm{mmol}$ ) according to GP $4 \mathrm{~A}$; reaction time: $2 \mathrm{~h}$; yield: $0.136 \mathrm{~g}(72 \%) ; R_{f}=0.55\left(10 \% \mathrm{MeOH}-\mathrm{CH}_{2} \mathrm{Cl}_{2}\right)$.

${ }^{1} \mathrm{H}$ NMR: $\delta=7.32-7.20(\mathrm{~m}, 5 \mathrm{H}), 2.86-2.82(\mathrm{~m}, 2 \mathrm{H}), 2.60-2.50(\mathrm{~m}, 2$ $\mathrm{H}), 2.40(\mathrm{~m}, 4 \mathrm{H}), 1.68-1.62(\mathrm{~m}, 4 \mathrm{H}), 1.51-1.48(\mathrm{~m}, 2 \mathrm{H})$.

${ }^{13} \mathrm{C}$ NMR: $\delta=140.7,128.7,128.3,125.9,61.5,54.6,33.7,26.1,24.5$.

Compound 10 was also prepared by reacting cyclopentene $(0.068 \mathrm{~g}$, $1 \mathrm{mmol})$ with phenethylamine (1 mmol) according to GP 4B; yield: $0.117 \mathrm{~g}(62 \%)$.

\section{9-(N-Morpholino)nonyl Acetate Ester (2d)}

Prepared from $2 a(0.099 \mathrm{~g}, 0.5 \mathrm{mmol})$ with morpholine $(1 \mathrm{mmol})$ by GP $4 \mathrm{~B}$; reaction time: $6 \mathrm{~h}$. The product was identical to $2 d$ obtained as above; yield: $0.089 \mathrm{~g}(66 \%)$.

N-Benzylcyclohexylmethanamine (11a) [4352-47-0] and N-benzylN-methylcyclohexylmethanamine (11b) [79952-95-7]

Prepared from the reaction of vinylcyclohexane $(0.11 \mathrm{~g}, 1 \mathrm{mmol})$ with benzylamine ( $2 \mathrm{mmol}$ ) by GP 4B; reaction time: 6 h; yield: 0.102 $\mathrm{g}(50 \%) ; R_{f}=0.50\left(10 \% \mathrm{MeOH}-\mathrm{CH}_{2} \mathrm{Cl}_{2}\right)$.

${ }^{1} \mathrm{H}$ NMR: $\delta=7.36-7.26(\mathrm{~m}, 5 \mathrm{H}), 3.80(\mathrm{~s}, 2 \mathrm{H}), 2.49(\mathrm{~d}, J=6.7 \mathrm{~Hz}, 2 \mathrm{H})$, 1.84-1.68 (m, $5 \mathrm{H}), 1.57-1.46(\mathrm{~m}, 1 \mathrm{H}), 1.32-1.13(\mathrm{~m}, 4 \mathrm{H}), 0.99-0.90(\mathrm{~m}$, $2 \mathrm{H})$.

${ }^{13} \mathrm{C}$ NMR: $\delta=140.6,128.4,128.0,126.8,56.2,54.1,37.9,31.5,26.7$, 26.1.

The reaction also yielded $10 \%$ of $11 b$.

$11 b$

$R_{f}=0.63\left(10 \% \mathrm{MeOH}-\mathrm{CH}_{2} \mathrm{Cl}_{2}\right)$.

${ }^{1} \mathrm{H}$ NMR (600 MHz): $\delta=7.35-7.24(\mathrm{~m}, 5 \mathrm{H}), 3.47$ (s, $\left.2 \mathrm{H}\right), 2.18-2.16$ $(\mathrm{m}, 5 \mathrm{H}), 1.84(\mathrm{~d}, J=12.4 \mathrm{~Hz}, 2 \mathrm{H}), 1.74-1.67(\mathrm{~m}, 3 \mathrm{H}), 1.57-1.51(\mathrm{~m}, 1$ $\mathrm{H}), 1.28-1.13(\mathrm{~m}, 3 \mathrm{H}), 0.89-0.82(\mathrm{~m}, 2 \mathrm{H})$.

${ }^{13} \mathrm{C}$ NMR $(150 \mathrm{MHz}): \delta=139.8,128.9,128.0,126.7,64.7,62.7,42.9$, $35.8,31.8,26.9,26.2$.

\section{3-Phenethyl-3-azabicyclo[3.2.1]octane (12)}

Prepared by reacting norbornene $(0.094 \mathrm{~g}, 1 \mathrm{mmol})$ with phenethylamine ( $1 \mathrm{mmol}$ ) according to GP 4A; reaction time: 2 h; yield: $0.139 \mathrm{~g}$ $(65 \%) ; R_{f}=0.63\left(10 \% \mathrm{MeOH}-\mathrm{CH}_{2} \mathrm{Cl}_{2}\right)$.
IR (neat): 2931, 2757, 1496, $1406 \mathrm{~cm}^{-1}$.

${ }^{1} \mathrm{H}$ NMR: $\delta=7.37-7.24(\mathrm{~m}, 5 \mathrm{H}), 2.84-2.80(\mathrm{~m}, 4 \mathrm{H}), 2.64-2.60(\mathrm{~m}, 2$ H), 2.20-2.14 (m, 4 H), 1.76-1.63 (m, 4 H), 1.56-1.53 (m, 1 H), 1.44-1.42 $(\mathrm{m}, 1 \mathrm{H})$.

${ }^{13} \mathrm{C}$ NMR: $\delta=140.9,128.8,128.2,125.8,60.2,60.1,37.7,35.2,33.3$, 28.6.

HRMS-FAB: $m / z$ calcd for $\mathrm{C}_{15} \mathrm{H}_{22} \mathrm{~N}(\mathrm{M}+\mathrm{H})^{+}$: 216.1747; found: 216.1748 .

This product was also prepared by reacting norbornene $(0.041 \mathrm{~g}$, $0.43 \mathrm{mmol})$ with phenethylamine $(0.43 \mathrm{mmol})$ according to GP $4 \mathrm{~B}$; yield: $0.062 \mathrm{~g}(65 \%)$.

1-Phenyl-2-(4-phenylbutan-2-yl)diazene (13) [343222-12-8]

A solution of alkene $4 a(0.176 \mathrm{~g}, 1 \mathrm{mmol})$ and $\mathrm{MeOH}(0.3 \mathrm{~mL})$ in $\mathrm{CH}_{2} \mathrm{Cl}_{2}(10 \mathrm{~mL})$ in a round-bottomed flask was cooled to $-40{ }^{\circ} \mathrm{C}$ and a stream of $2 \% \mathrm{O}_{3} / \mathrm{O}_{2}$ (approximately $1 \mathrm{mmol} \mathrm{O}_{3} / \mathrm{min}$ ) was bubbled through the reaction solution. Once the pale blue color of ozone was observed, the ozonizer voltage was set to zero and the reaction was sparged for 1 min with $\mathrm{N}_{2}$. The resulting solution was removed from dry ice/acetone bath, whereupon $\mathrm{NaBH}(\mathrm{OAc})_{3}(2 \mathrm{mmol})$ and phenylhydrazine $(1 \mathrm{mmol})$ were sequentially added. The reaction was stirred for $2 \mathrm{~h}$ at r.t. and then treated with $\mathrm{NaCNBH}_{3}(1 \mathrm{mmol})$. After stirring for another $30 \mathrm{~min}$, the reaction was filtered though neutral alumina with $\mathrm{CH}_{2} \mathrm{Cl}_{2}$, and the residue obtained upon concentration was purified by chromatography (alumina) using 10\% EtOAc-hexanes to furnish a light yellow oil, which darkened very rapidly. Air was passed over the oil for $2 \mathrm{~h}$ to furnish diazene 13 as a yellow oil; yield: $0.135 \mathrm{~g}(57 \%) ; R_{f}=0.50$ (10\% EtOAc-hexanes).

${ }^{1} \mathrm{H}$ NMR: $\delta=7.73-7.70(\mathrm{~m}, 2 \mathrm{H}), 7.53-7.46(\mathrm{~m}, 3 \mathrm{H}), 7.33-7.28(\mathrm{~m}, 2$ $\mathrm{H})$, 7.24-7.20 (m, $3 \mathrm{H}), 3.90-3.81(\mathrm{~m}, 1 \mathrm{H}), 2.66(\mathrm{t}, J=8.1 \mathrm{~Hz}, 2 \mathrm{H})$, 2.41-2.32 (m, $1 \mathrm{H}), 2.13-2.04(\mathrm{~m}, 1 \mathrm{H}), 1.42(\mathrm{~d}, J=6.6 \mathrm{~Hz}, 3 \mathrm{H})$.

${ }^{13} \mathrm{C}$ NMR: $\delta=152.1,141.9,130.3,129.0,128.5,128.4,125.8,122.2,72.7$, $36.9,32.6,19.0$.

Supporting Information for this article follows the References.

Acknowledgments - Research was conducted with NSF funding (CHE-0749916, -1057982) in facilities remodeled with NIH support (RR016544). NMR spectra were acquired, in part, on instruments purchased with NSF support (MRI 0079750 and CHE 0091975).

\section{References}

1. Authors Kyasa and Fisher contributed equally to this article.

2. a.) Margaretha P, In Science of Synthesis Vol. 40a: Schaumann E, Enders D, Thieme; Stuttgart: 2009. p. 65-89. b. Baxter EW, Reitz AB, Org. React. 2002, 59: 1. c.) Ghosh AK, Bilcer G, Schiltz G, Synthesis 2001, 2203.

3. Van Ornum SG, Champeau RM, Pariza R, Chem. Rev. 2006, 106: 2990

4. Although a variety of reagents have been applied for post-ozonolysis reductions, most examples employ $\mathrm{Me}_{2} \mathrm{~S}, \mathrm{Ph}_{3} \mathrm{P}$, thiourea, or $\mathrm{Zn} /$ AcOH: a.) Kropf H, In Houben-Weyl Methoden der Organischen Chemie, Peroxo-Verbindungen Kropf H, Thieme; Stuttgart: 1988. p.11021116 . b.) The reduction of ozonides, tetraoxanes, or polymeric peroxides derived from hindered substrates can be very slow, leading to the inadvertent concentration of potentially explosive intermediates, see, for example: Chen L, Wiemer DF, J. Org. Chem. 2002, 67: 7561. c.) Lavalle P, Bouthillier G, J. Org. Chem. 1986, 51: 1362. d.) Hida T, Kikuchi J, Kakinuma M, Nogusa H, Org. Process Res. Dev. 2010, 14: 1493. e.) Gordon PM, Chem. Eng. News 1990, 68 (32): 2. 
5. a.) Fisher TJ, Dussault PH, Tetrahedron Lett. 2010, 51: 5615. b.) Schiaffo CE, Dussault PH, J. Org. Chem. 2008, 73: 4688. c.) Schwartz C, Raible J, Mott K, Dussault PH, Tetrahedron 2006, 62: 10747.

6. a.) Abdel-Magid AF, Mehrman SJ, Org. Process Res. Dev. 2006, 10: 971. b.) Abdel-Magid AF, Carson KC, Harris BD, Maryanoff CA, Shah RD, J. Org. Chem. 1996, 61: 3849. c.) Gribble G, Chem. Soc. Rev. 1998, 27: 395. d.) Matos K, Pichlmair S, Burkhardt ER, Chim. Oggi. 2007, 25: 17.

7. Lane CF, Synthesis 1975, 135.

8. For reductive aminations involving $\mathrm{NaCNBH}_{3}$ and hydroperoxyacetals or related intermediates, see: a.) Namba K, Murata Y, Horikawa M, Iwashita T, Kusumoto S, Angew. Chem. Int. Ed. 2007, 46: 7060. b.) Shawe TT, Sheils CJ, Gray SM, Conard JL, J. Org. Chem. 1994, 59: 5841. c.) Kawaguchi M, Hayashi O, Sakai N, Hamada M, Yamamoto Y, Oda J, Agric. Biol. Chem. 1986, 50: 3107.

9. Ozonides react with $\mathrm{NaCNBH}_{3}$ very slowly, as evidenced by the selective reduction of a ketone in the presence of an ozonide: Laventine DM, Davies M, Evinson EL, Jenkins PR, Cullis PM, Fawcett J, Tetrahedron Lett. 2005, 46: 307.

10. Ozonides derived from mono- and 1, 1-disubstituted alkenes can react with secondary amines to form tertiary amines through basepromoted $\mathrm{E}_{1} \mathrm{cb}$ fragmentation to form an aldehyde, which undergoes reductive amination in the presence of the cogenerated formate: Hon Y.-S, Lu L, Tetrahedron Lett. 1993, 34: 5309.
11. Reductive amination of ozonides and/or hydroperoxyacetals has been accomplished using more powerful reducing systems; Raney Ni: a.) Pollart KA, Miller RE, J. Org. Chem. 1962, 27: 2392. b.) Diaper DGM, Mitchell DL, Can. J. Chem. 1962, 40: 1189. c.) White RW, King SW, O'Brien JL, Tetrahedron Lett. 1971, 3591. Pt/ $\mathrm{H}_{2}$ : d.) Brooks PR, Caron S, Coe JW, Ng KK, Singer RA, Vazquez E, Vetelino MG, Watson HH, Whritenour DC, Wirtz MC, Synthesis 2004, 1755. $\mathrm{NaBH}_{4}$ : e.) Mustafin AG, Dyachenko DI, Gataullin RR, Ishmuratov GYu, Kharisov RYa, Abdrakhmanov IB, Tolstikov GA, Russ. Chem. Bull. 2003, 52: 989.

12. a.) Noe MC, Hawkins JM, Snow SL, Wolf-Gouveia L, J. Org. Chem. 2008, 73: 3295. b.) Yang L, Butora G, Jiao RX, Pasternak A, Zhou C, Parsons WH, Mills SG, Vicario PP, Ayala JM, Cascieri MA, MacCoss M, J. Med. Chem. 2007, 50: 2609.

13. $\mathrm{NaBH}(\mathrm{OAc})_{3}$ has been reported to reduce ozonolysis products to the corresponding alcohols: Ishmuratov GYu, Kharisov RYa, Yakovleva MP, Botsman OV, Muslukhov RR, Tolstikov GA, Russ. J. Org. Chem. 2001, 37: 37

14. a.) Bunnelle WH, Chem. Rev. 1991, 91: 335. b.) Zmitek K, Zupan M, Iskra J, Org. Biomol. Chem. 2007, 5: 3895.

15. All products were purified by flash chromatography to greater than $95 \%$ purity (NMR). Hydroperoxides were characterized by ${ }^{1} \mathrm{H} /{ }^{13} \mathrm{C}$ NMR; aldehydes and amines were characterized by ${ }^{1} \mathrm{H} /{ }^{13} \mathrm{C}$ NMR, IR, and HRMS.

16. Smith LL, Hill FL, J. Chromatogr. 1972, 66: 101. 
Supporting Information: A Mild One-pot Conversion of Alkenes to Amines through Tandem Ozonolysis and Reductive Amination.

\section{ShivaKumar Kyasa ${ }^{1}$, Thomas J. Fisher ${ }^{1}$, and Patrick H. Dussault*}

Department of Chemistry, University of Nebraska-Lincoln, Lincoln, NE 68588-0304

$$
\text { pdussault1@unl.edu }
$$

\section{Table of Contents}

\section{Preparation of hydroperoxyacetals}

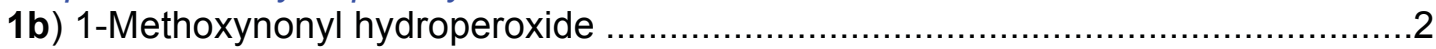

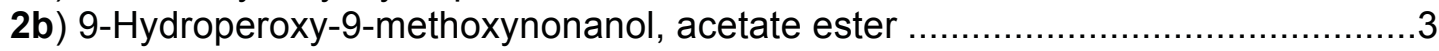

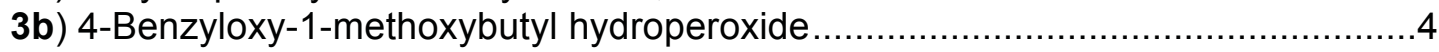

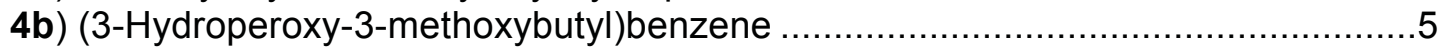

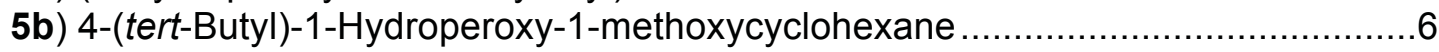

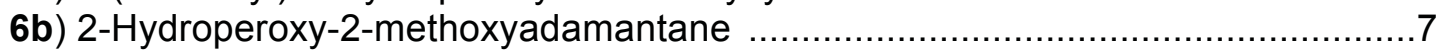

Synthesis of carbonyls from hydroperoxy acetals:

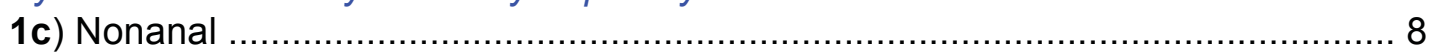

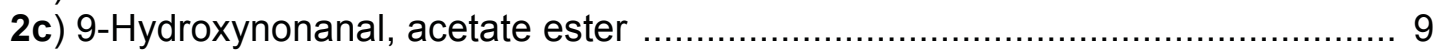

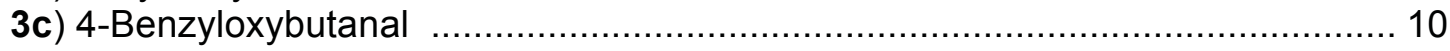

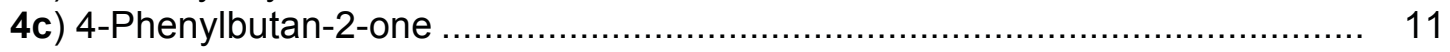

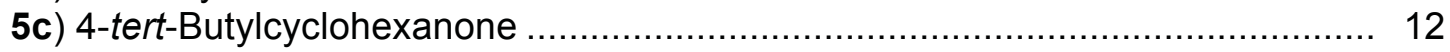

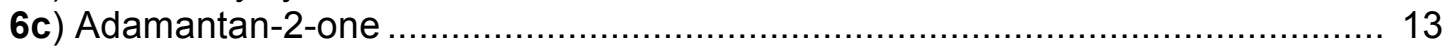

Synthesis of amines from hydroperoxy acetals:

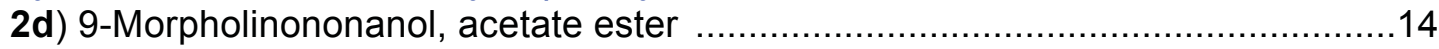

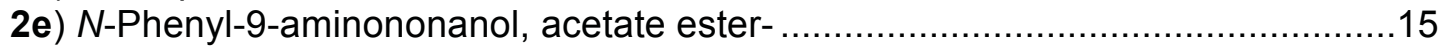

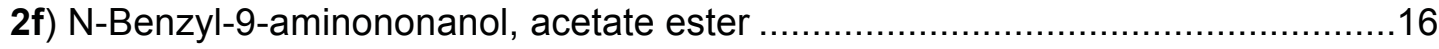

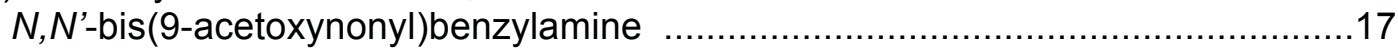

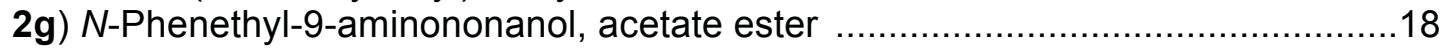

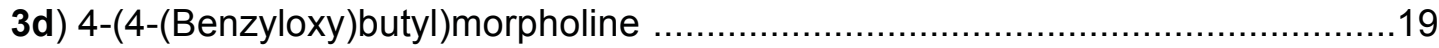

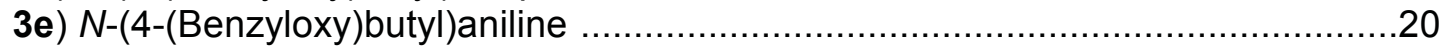

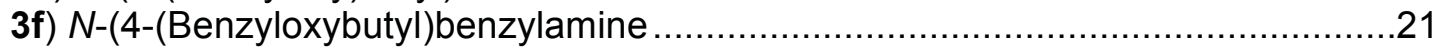

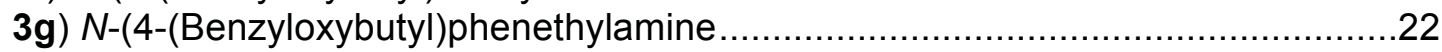

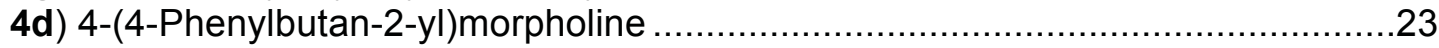

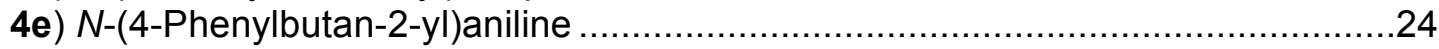

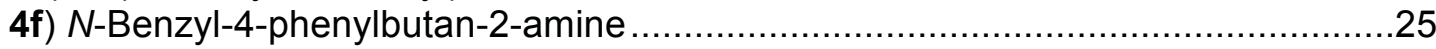

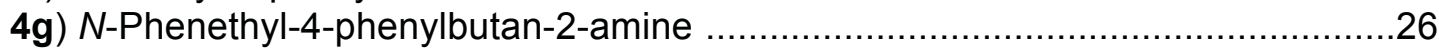

Synthesis of amines from alkenes:

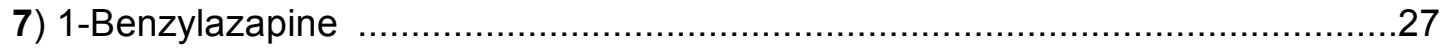

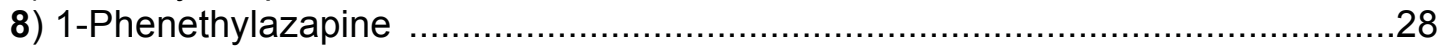

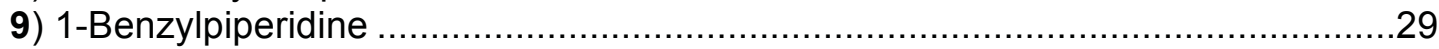

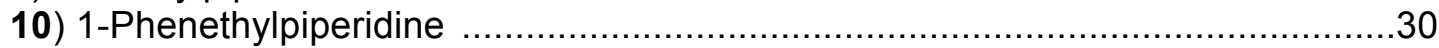

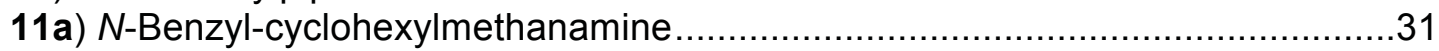

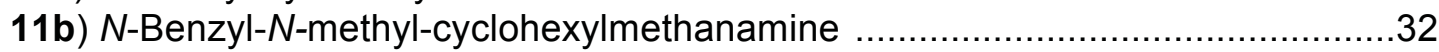

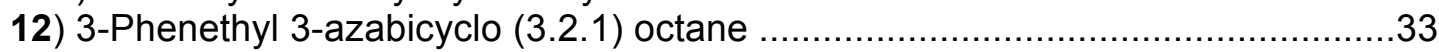

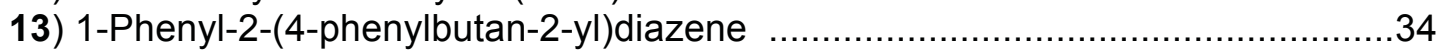




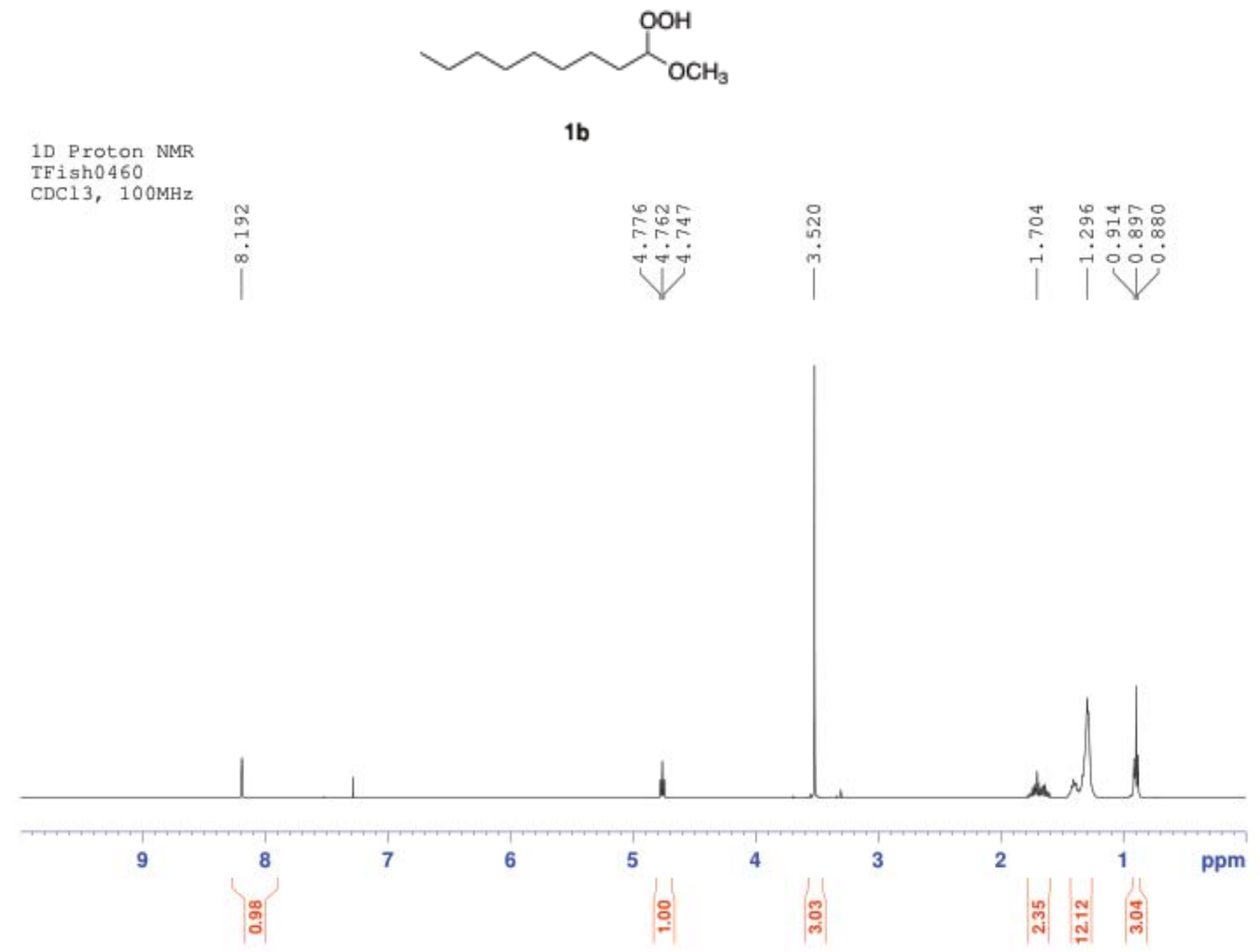

$13 \mathrm{C}$

sh0 460

CDCl3, $100 \mathrm{MHz}$
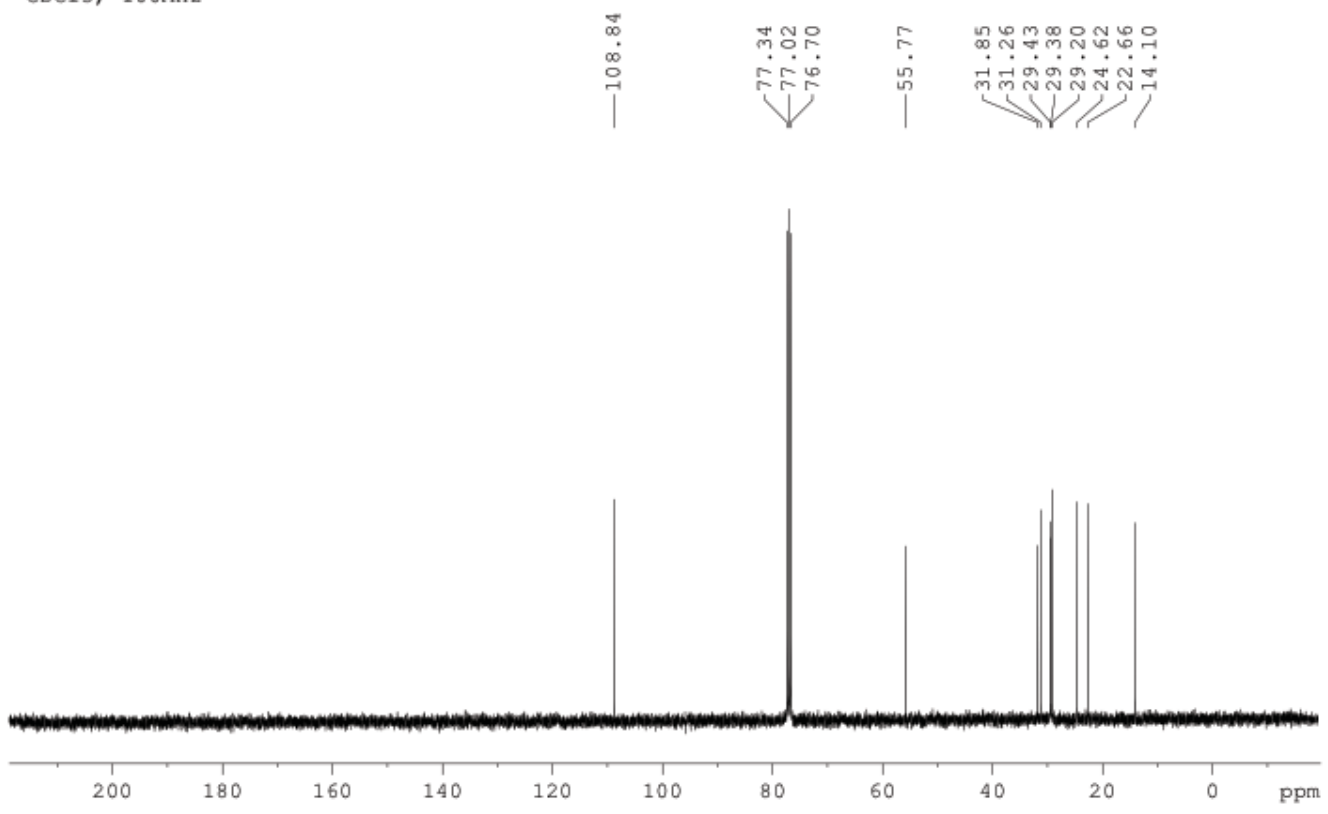


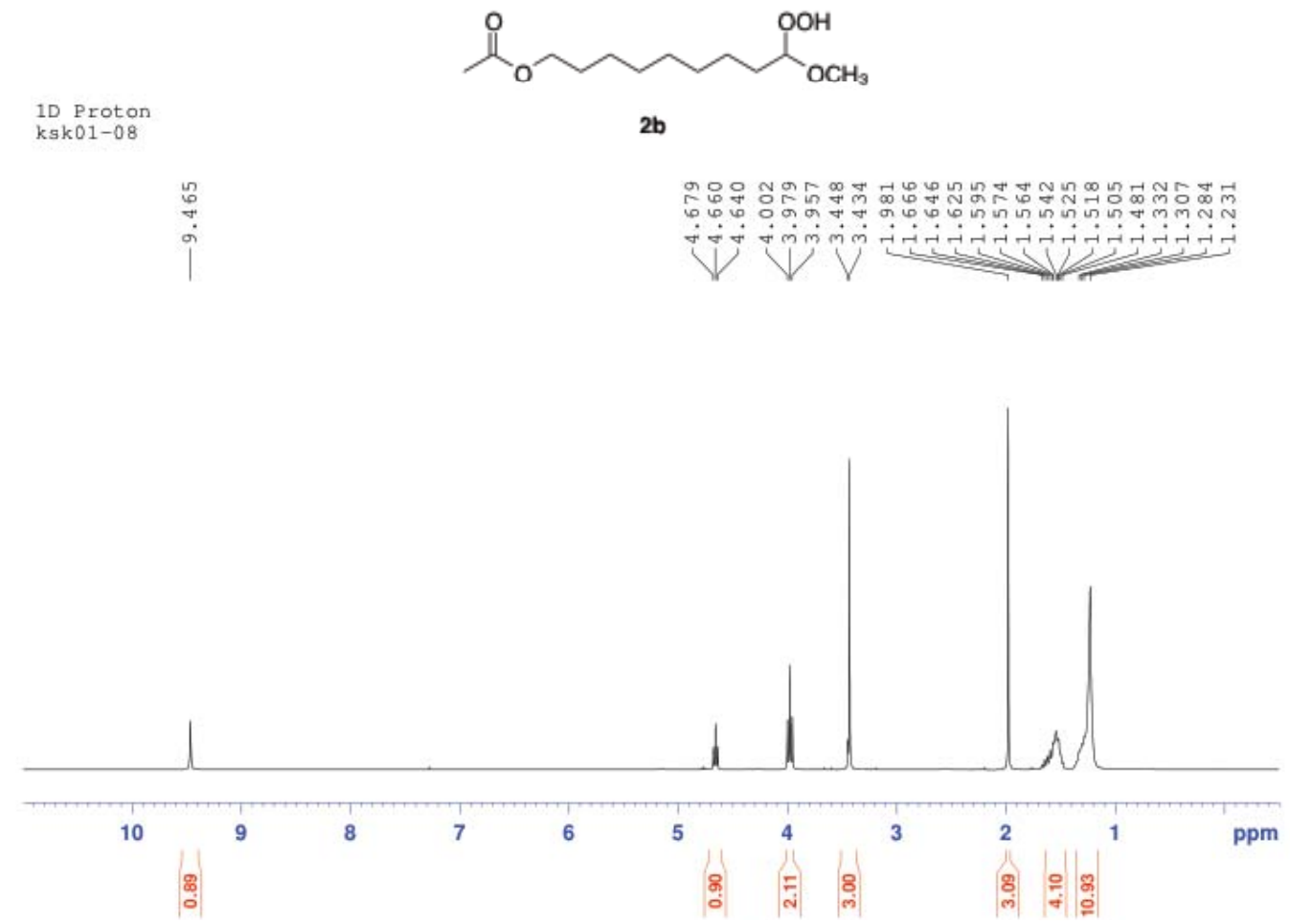

$13 \mathrm{C}$
ksk01-08
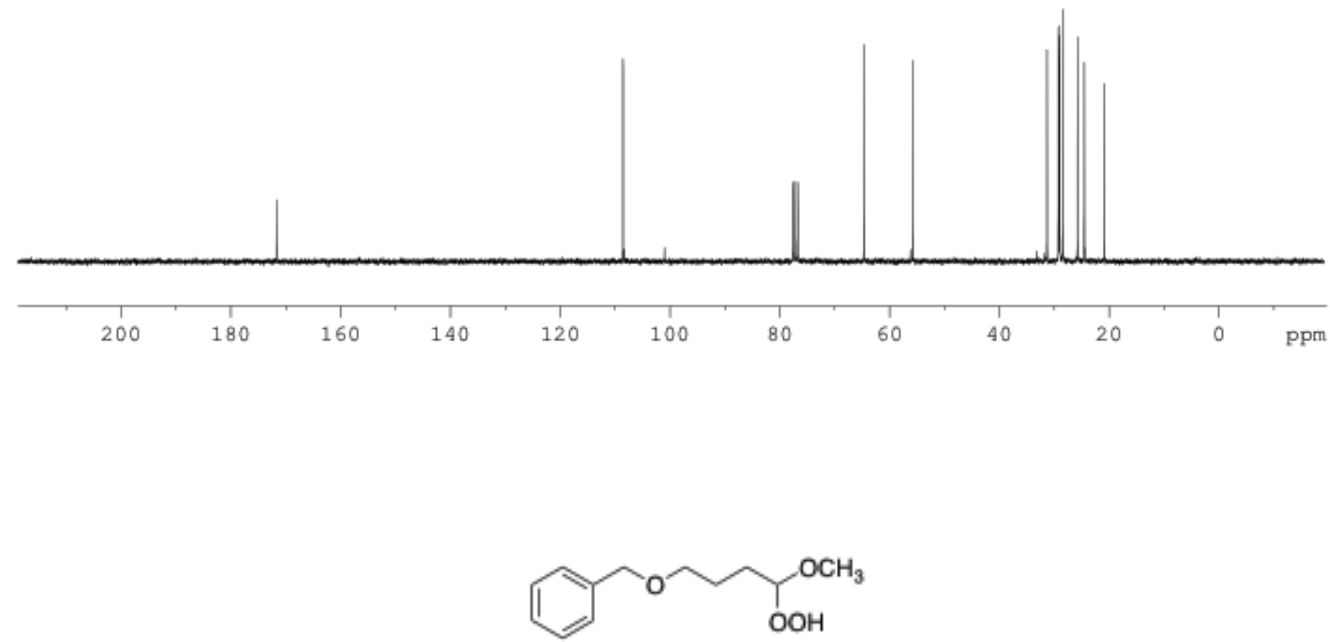

$3 b$ 
1D Proton NMR

ksk01-94

$400 \mathrm{MHz}, \mathrm{CDCl} 13$

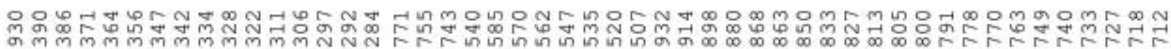

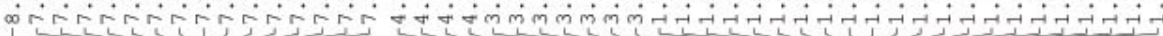

1 .

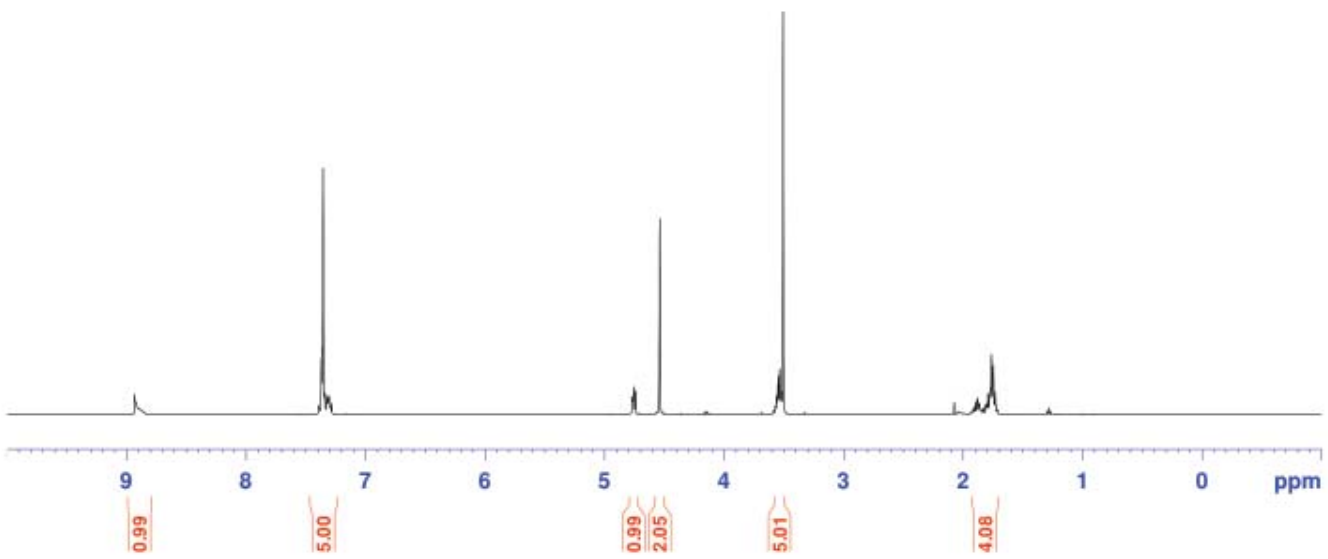

$13 \mathrm{C}$

ksk01-94

$\mathrm{CDCl} 3,400 \mathrm{MHz}$
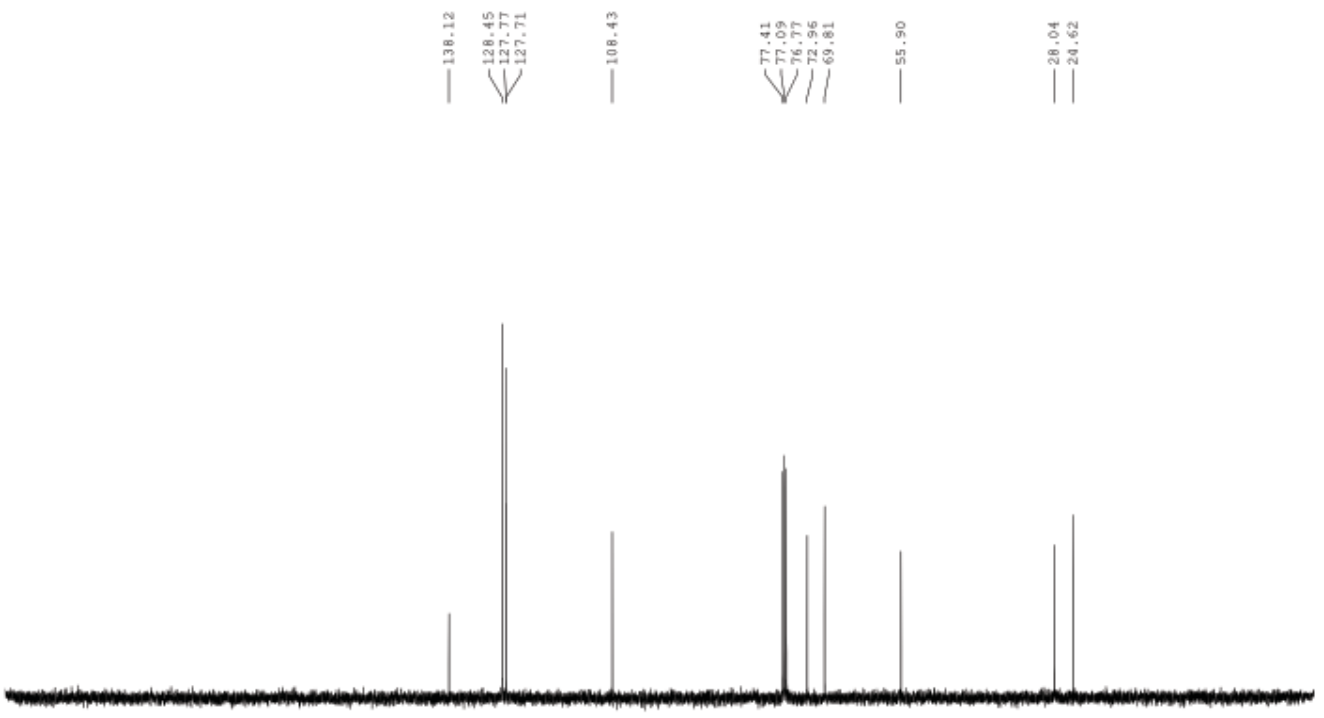

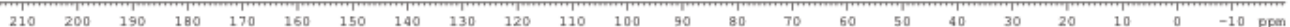

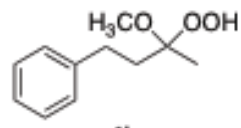

$4 b$ 
1D Proton NMR ksk01-79

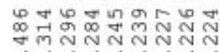

$\therefore r i r i r$

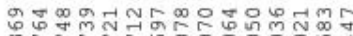

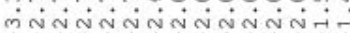

$\underbrace{2}$

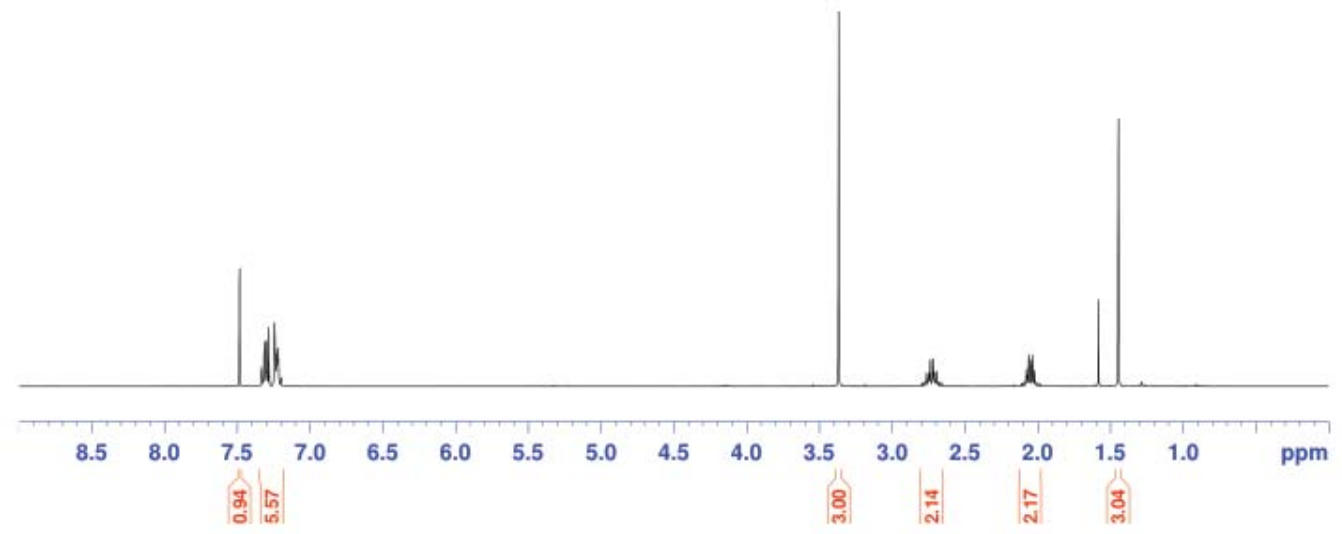

$13 \mathrm{C}$

ksk01-79
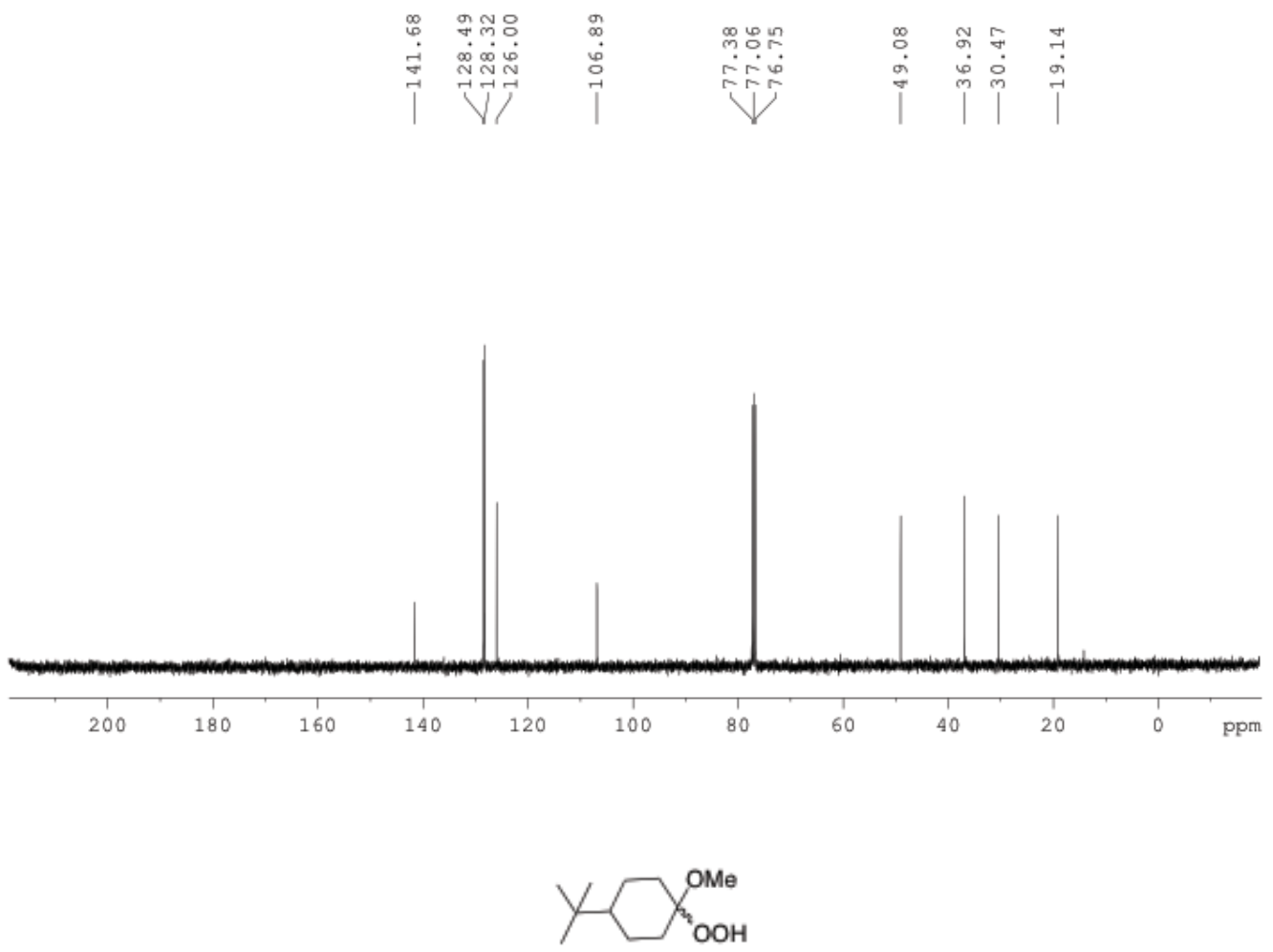

$5 \mathrm{~b}$ 
1D Proton NMR

CDCl3, 400MH z

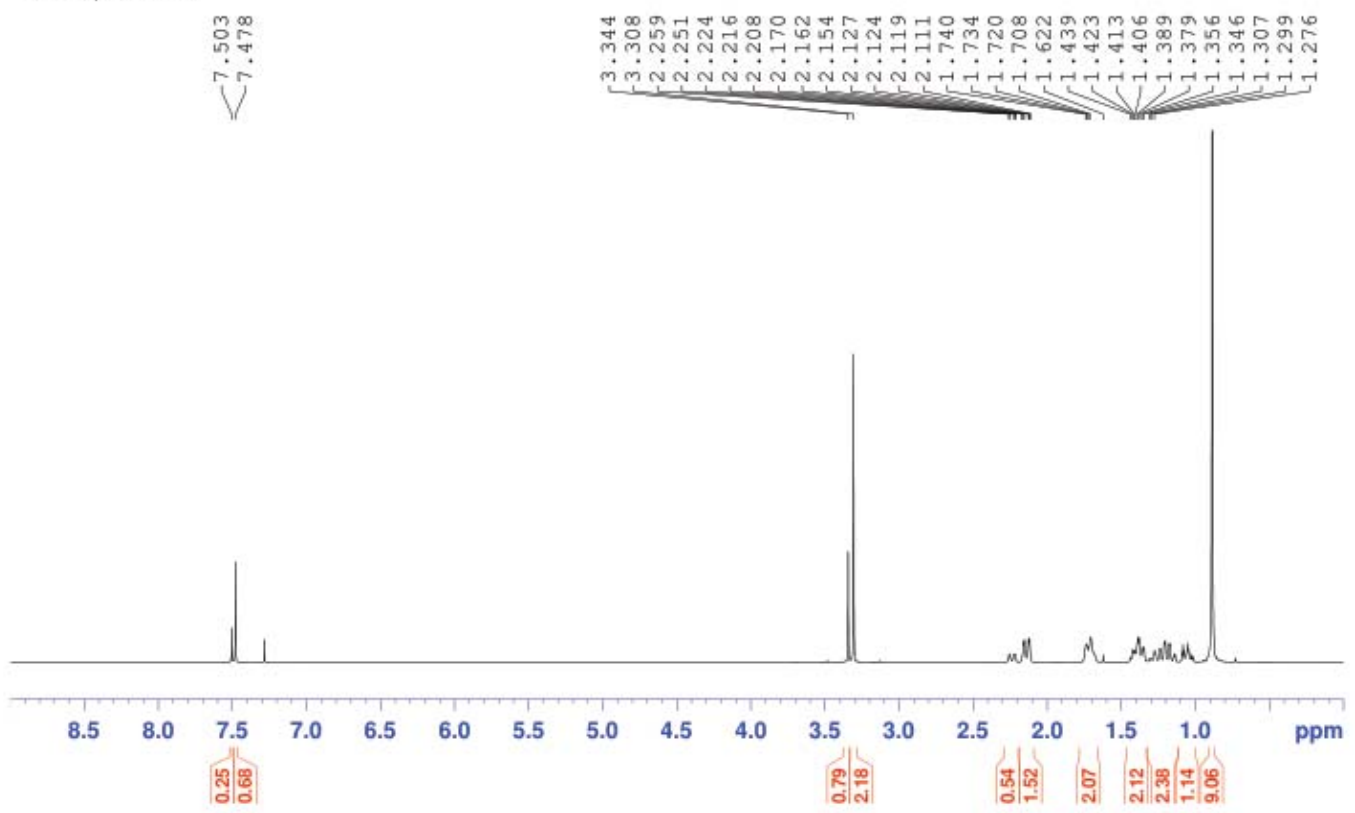

$13 \mathrm{C}$

TFish 0580

CDCl3, 400MHz
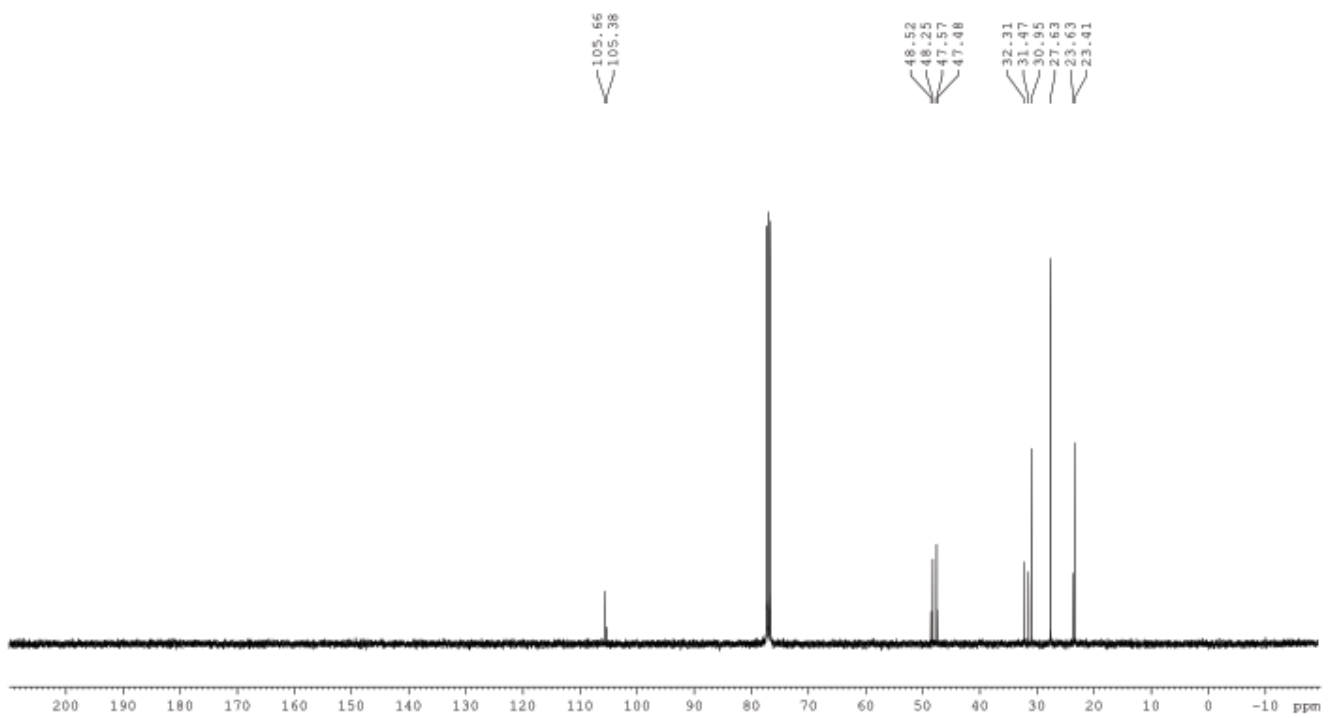
1D Proton NMR

KSKO1-77

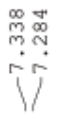

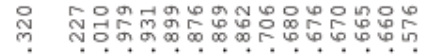

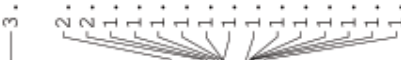

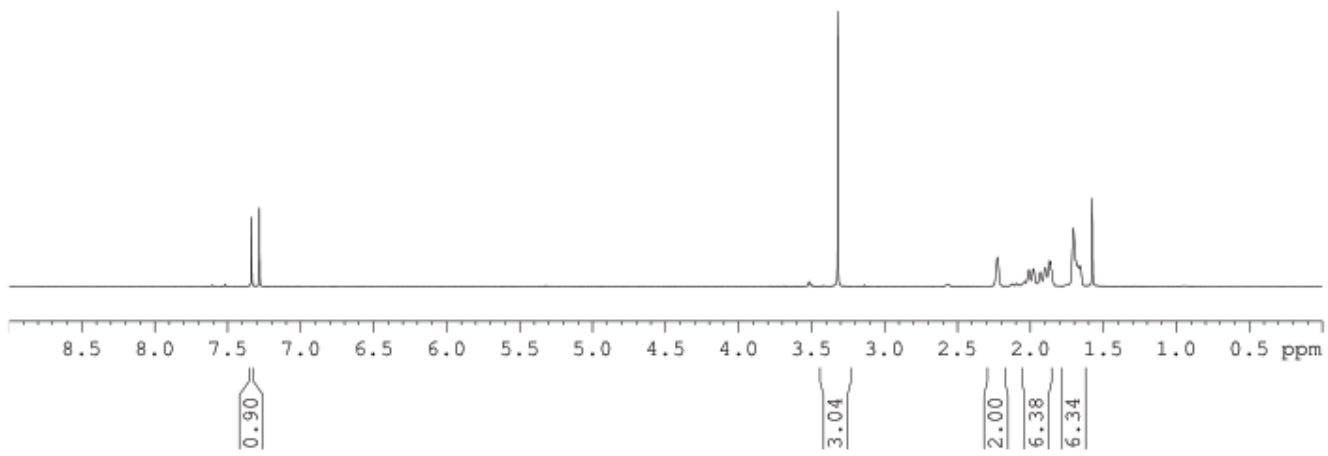

$13 \mathrm{C}$

KSK01-77
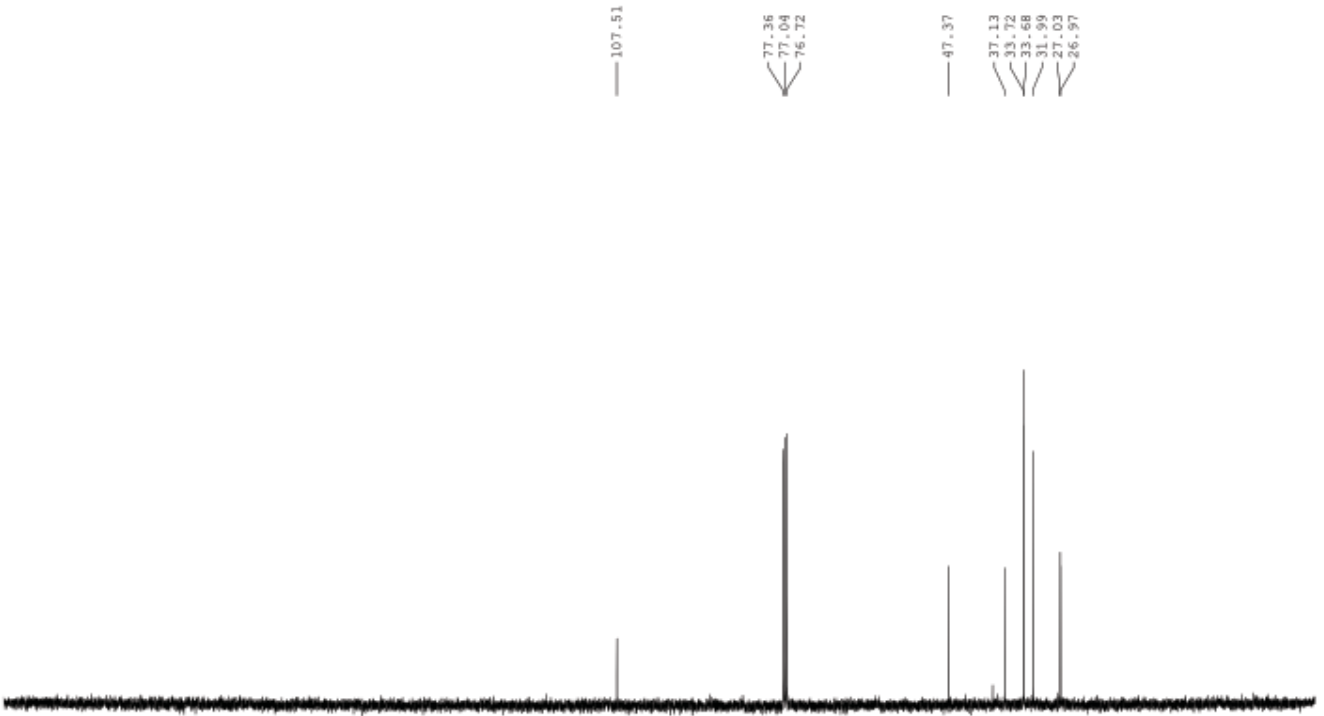

\begin{tabular}{lllllllllllllllllllllll}
120 & 200 & 190 & 180 & 170 & 160 & 150 & 140 & 130 & 120 & 110 & 100 & 90 & 80 & 70 & 60 & 50 & 40 & 30 & 20 & 10 & 0 & -10 \\
\hline
\end{tabular}

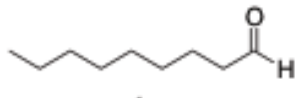

$1 c$ 
1D Proton NMR

ksk01-66
CDCl3, $400 \mathrm{MHz}$
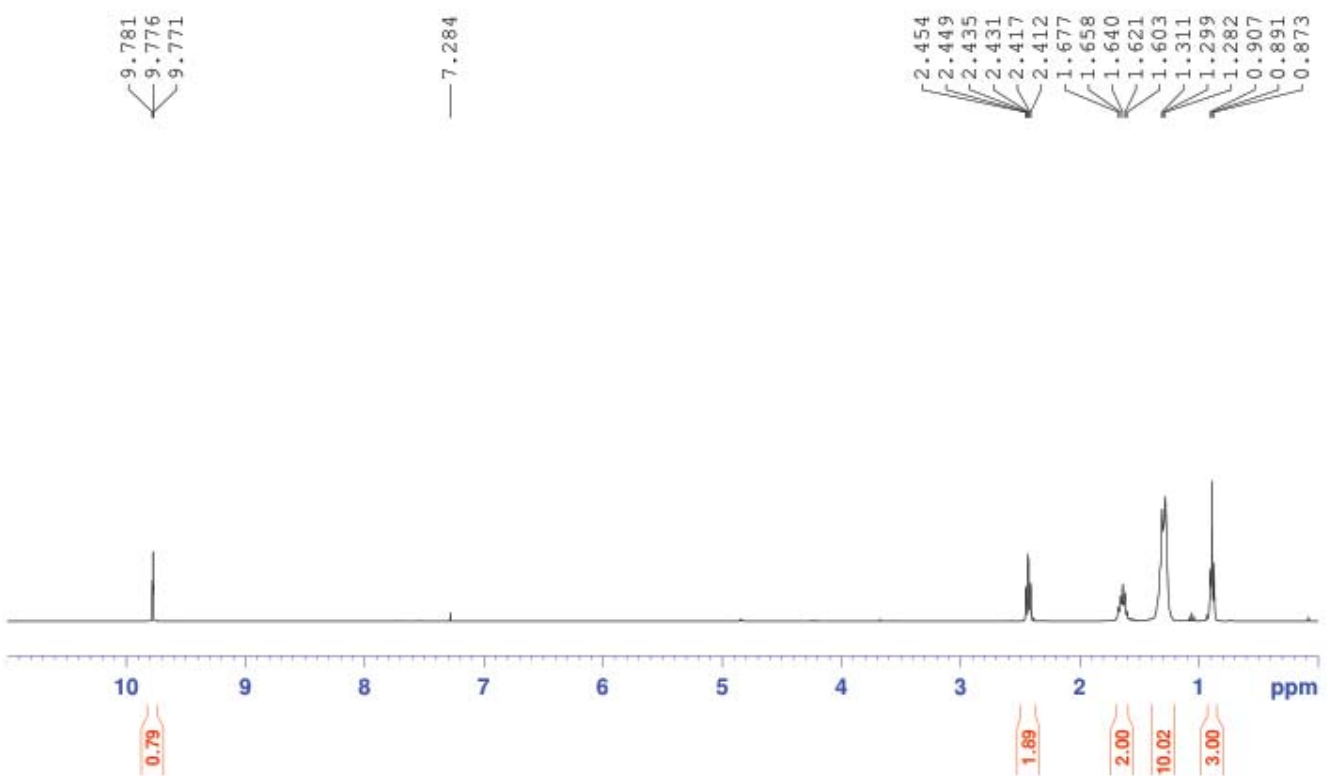

$13 \mathrm{C}$

ksk01-66

CDC13, $400 \mathrm{MHz}$
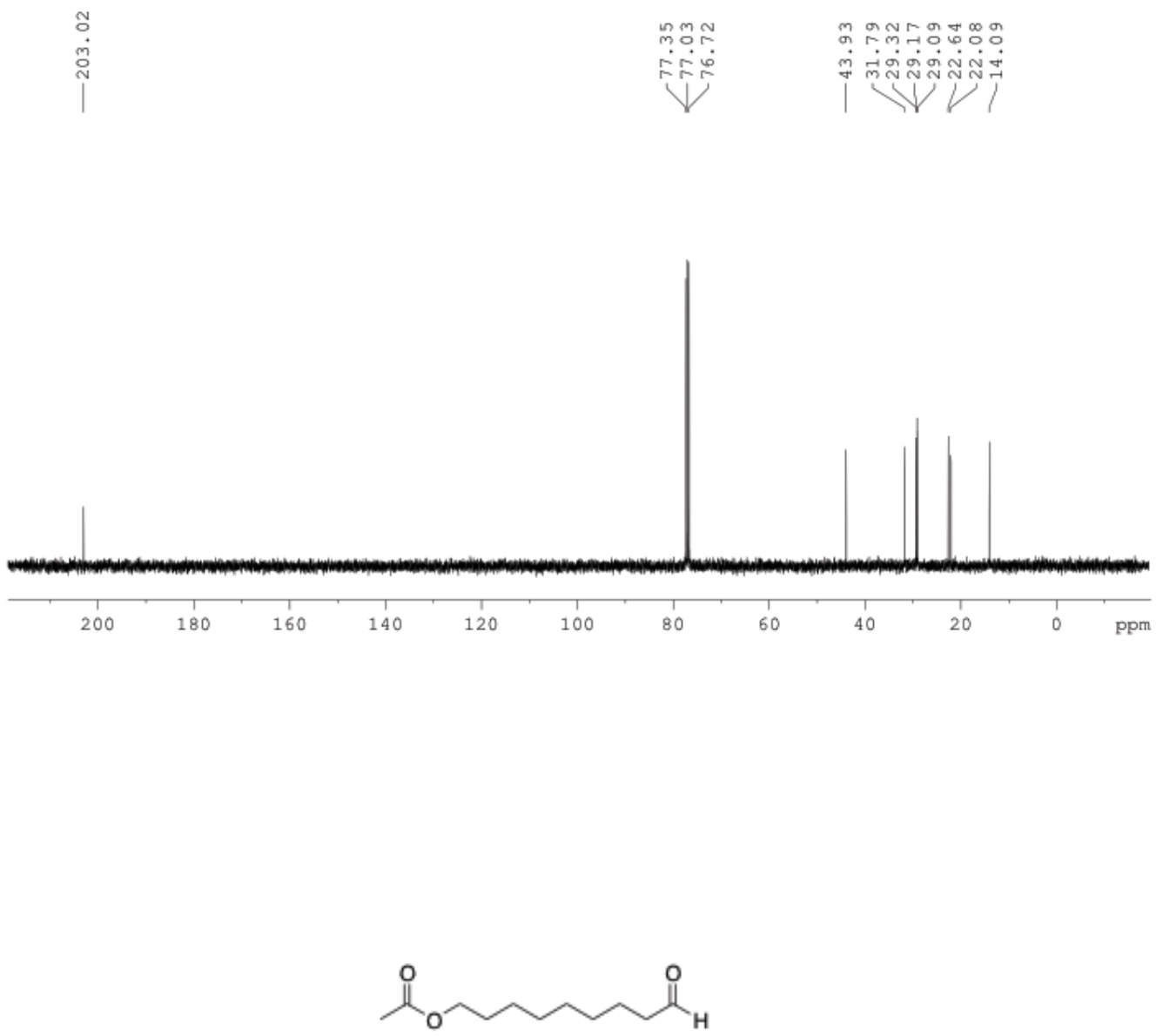

2c 
1D Proton NMR

ksk01-20
CDC13, $400 \mathrm{MHz}$
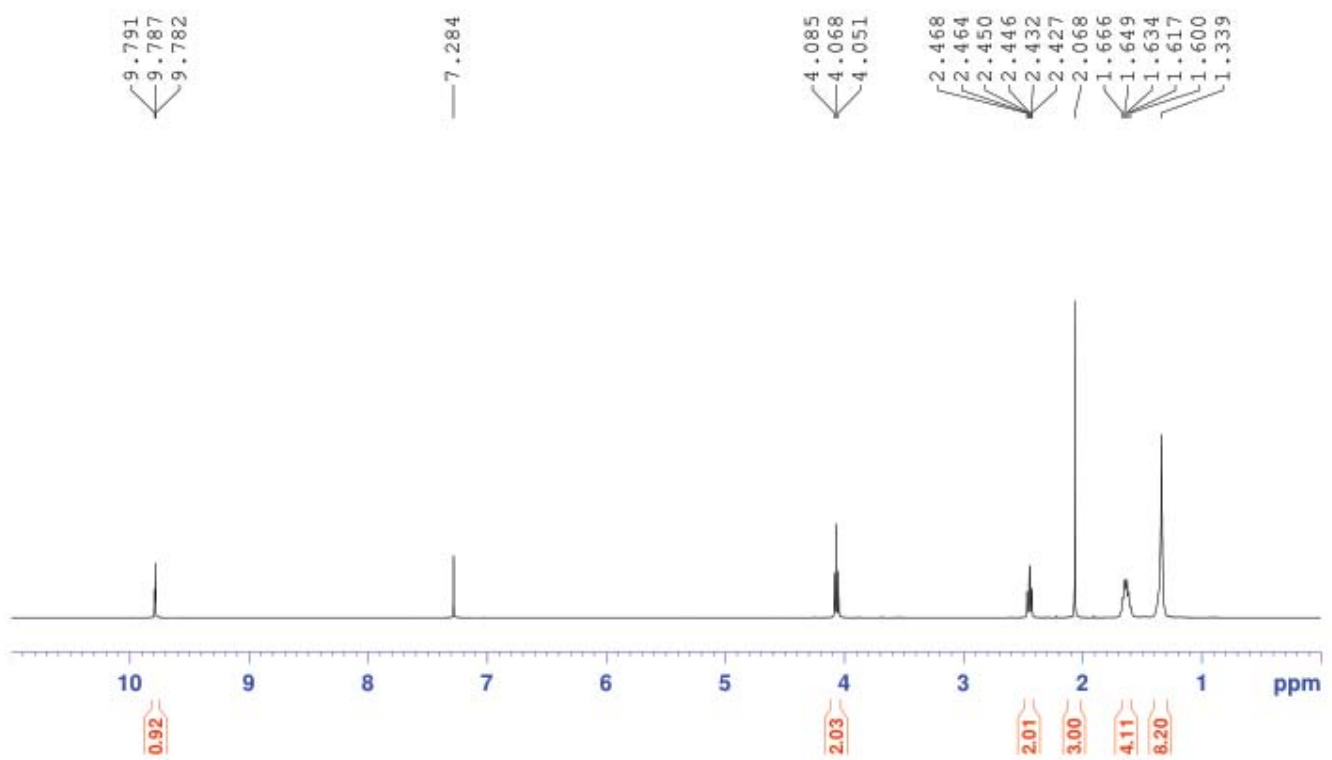

$13 \mathrm{C}$

ksk01-20
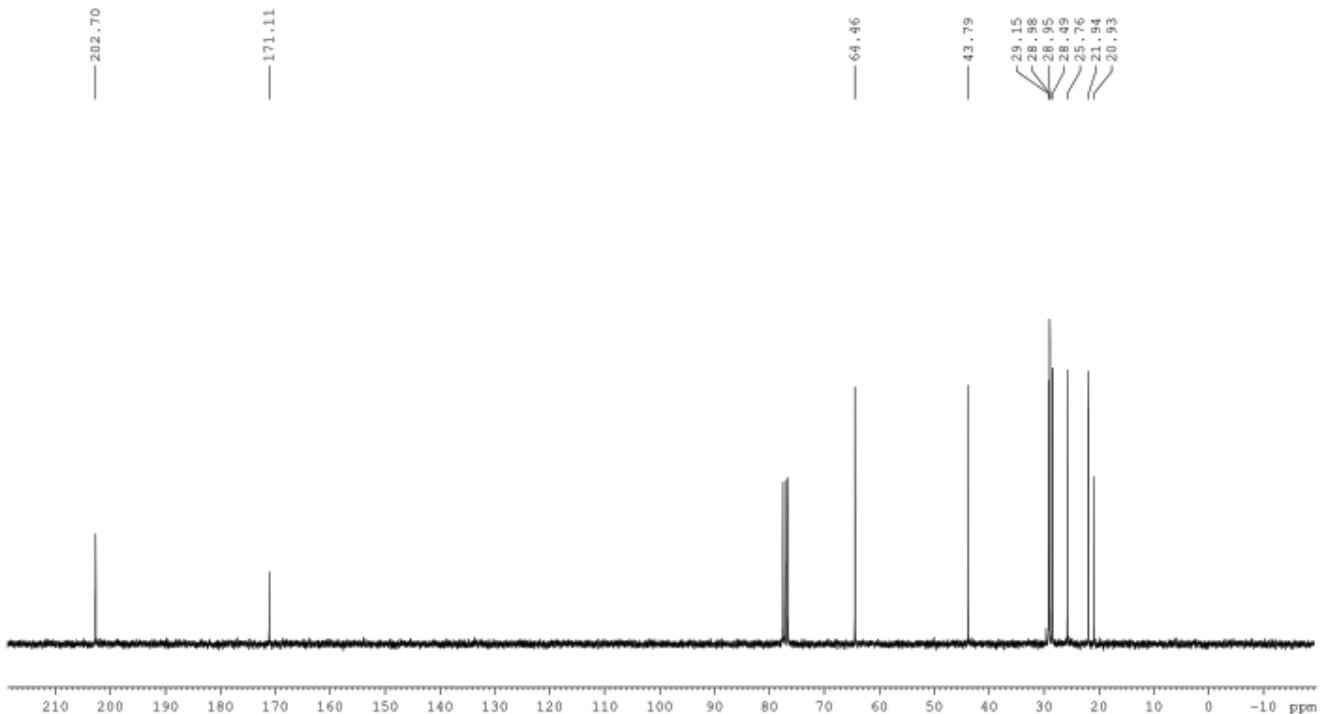

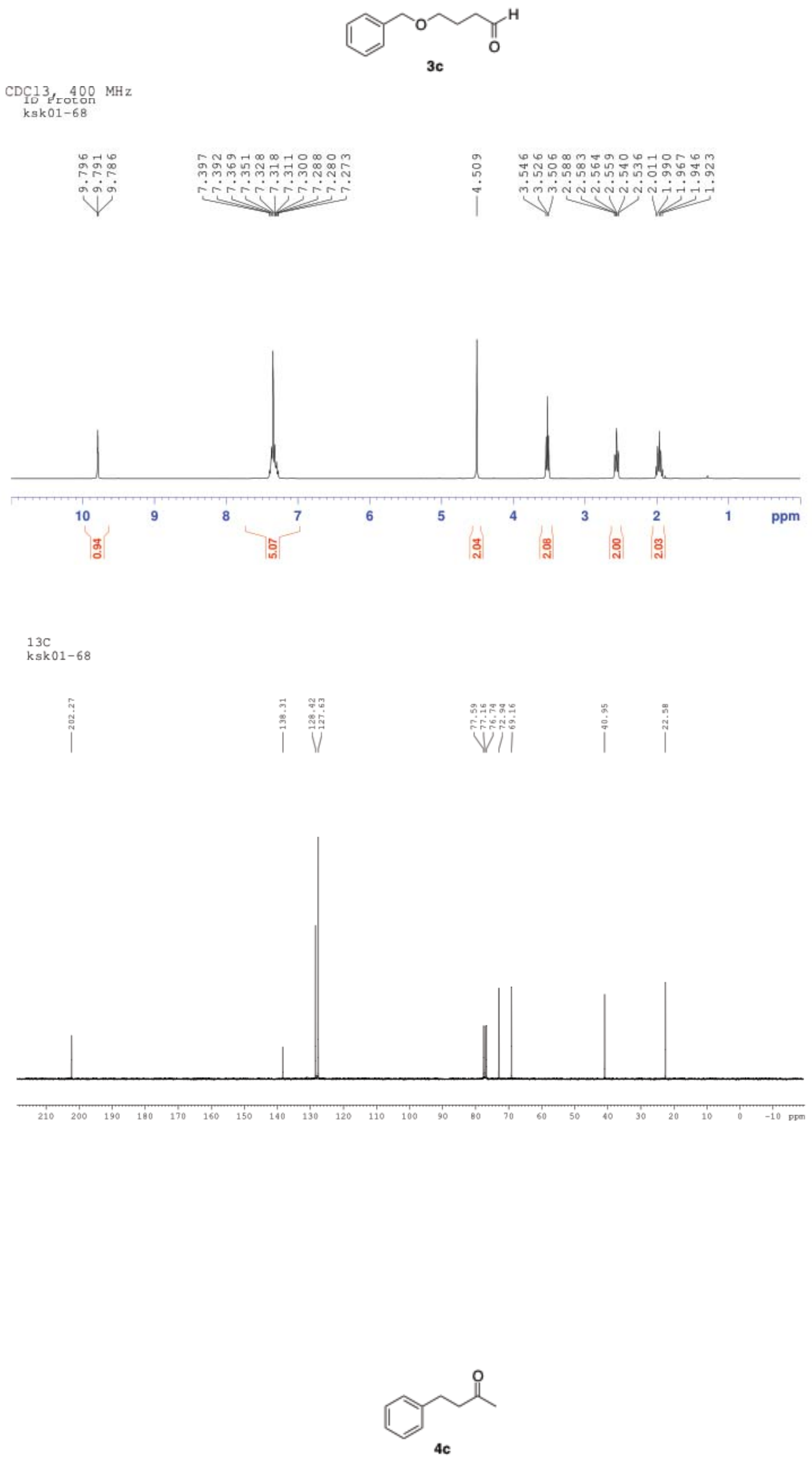
1D Proton NMR

ksk01-80

$\mathrm{CDCl} 3,400 \mathrm{MHz}$

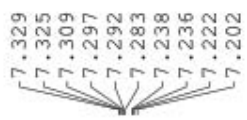

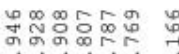

Ninis

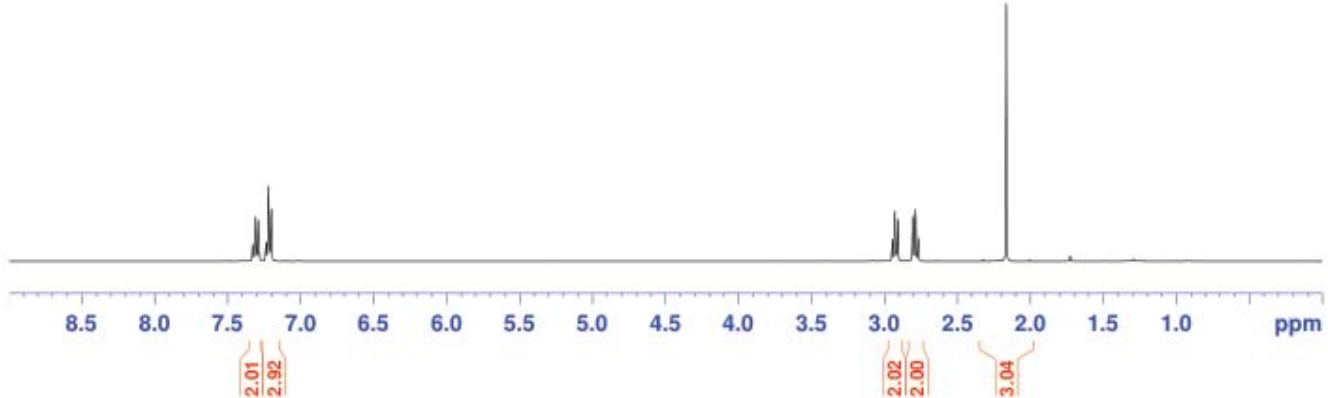

$13 \mathrm{C}$

ksk01-80

مै
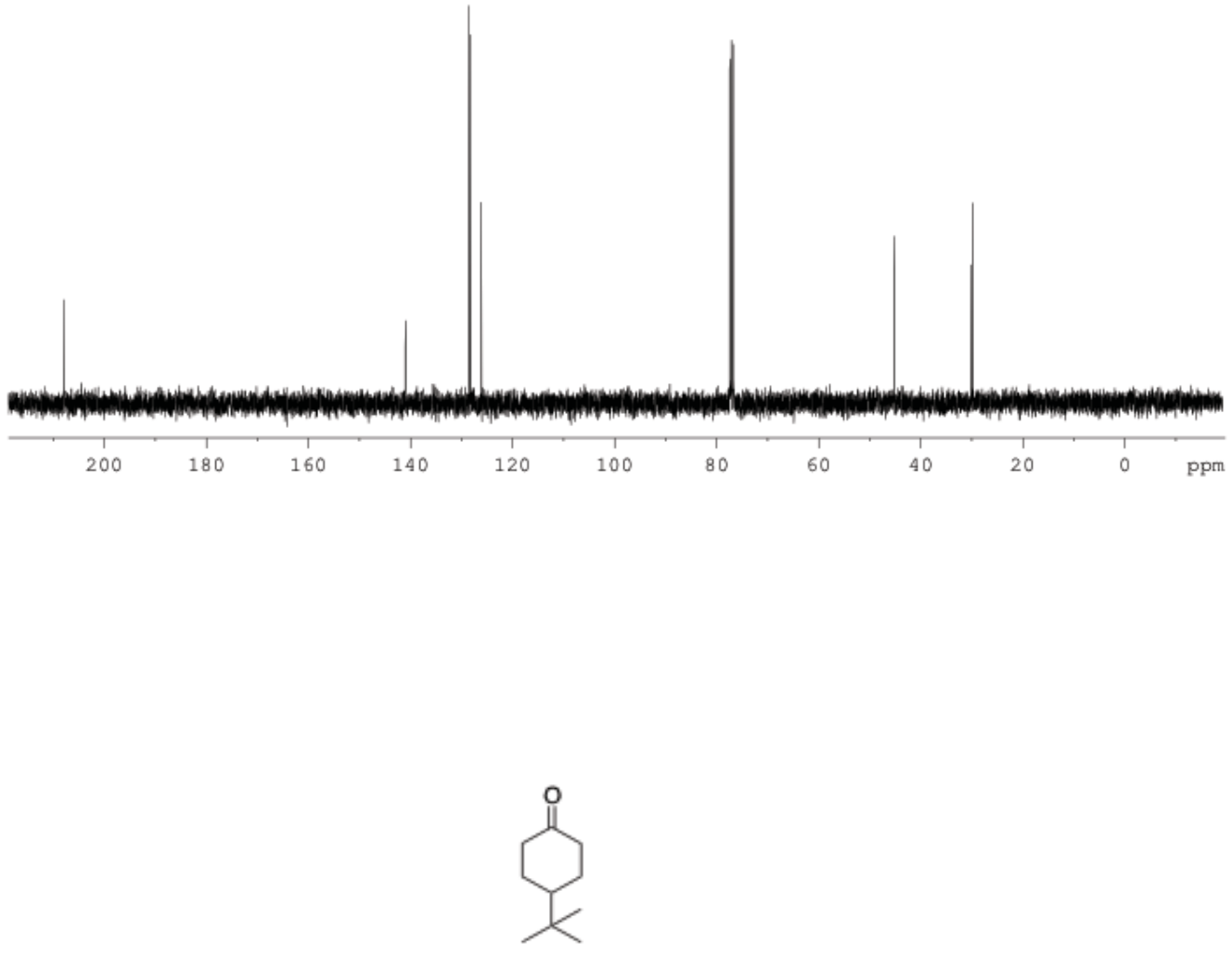
(
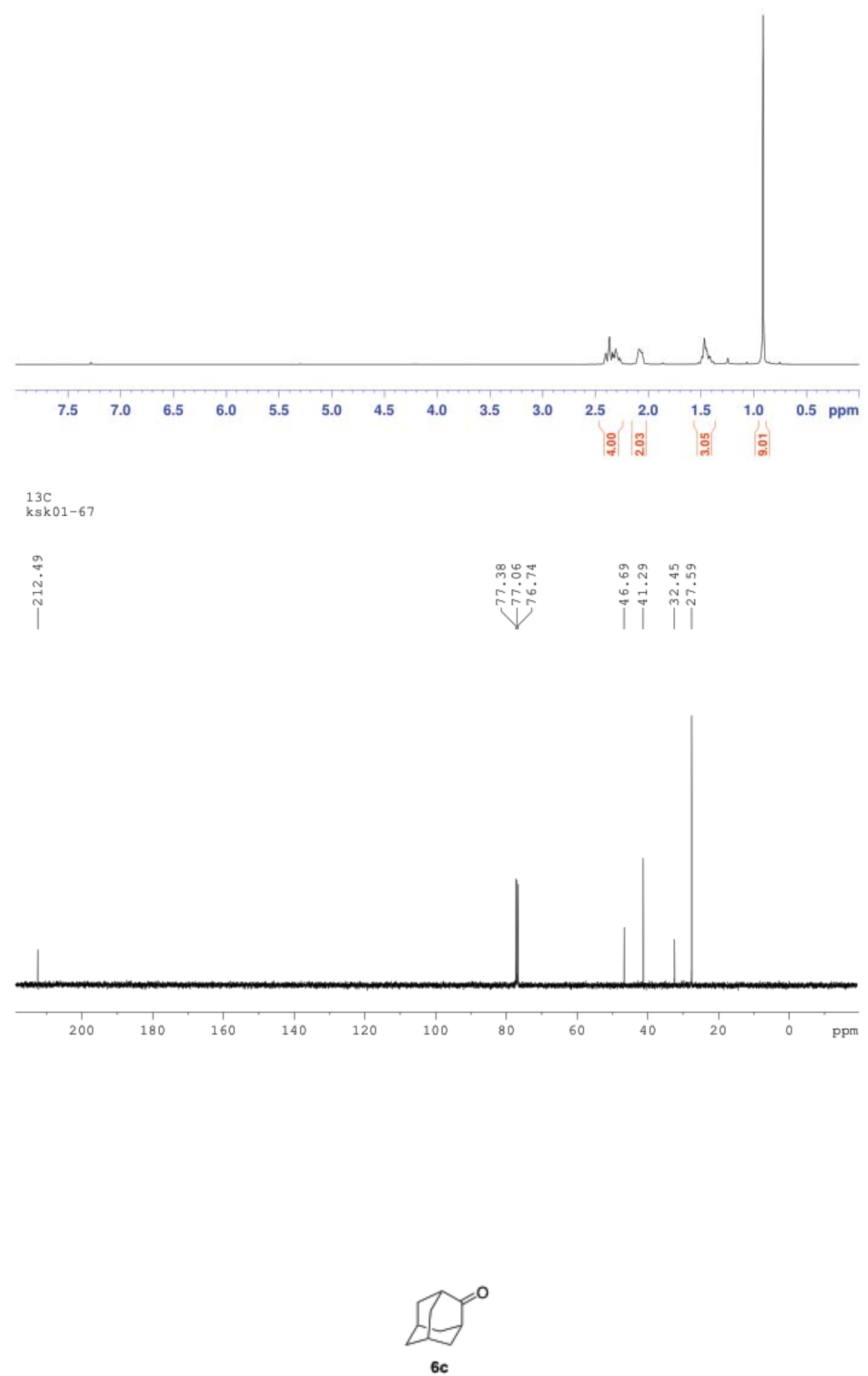
1D Proton NMR

ksk01-78

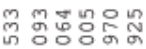

N N

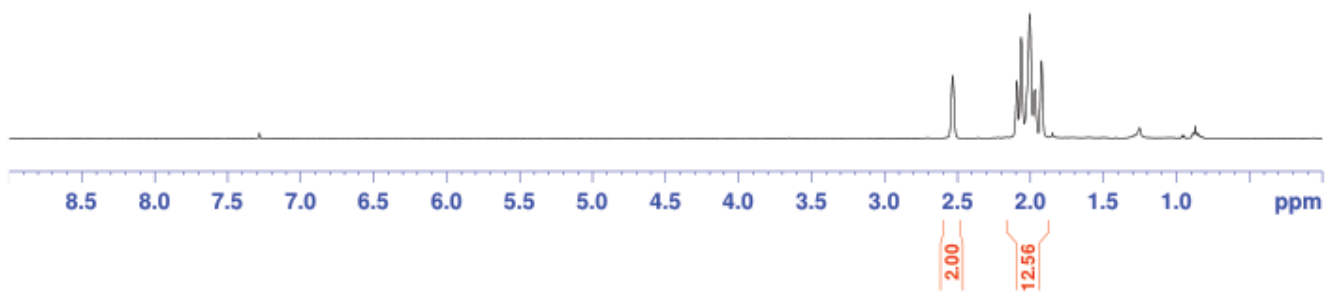

$13 \mathrm{C}$

ksk01-78
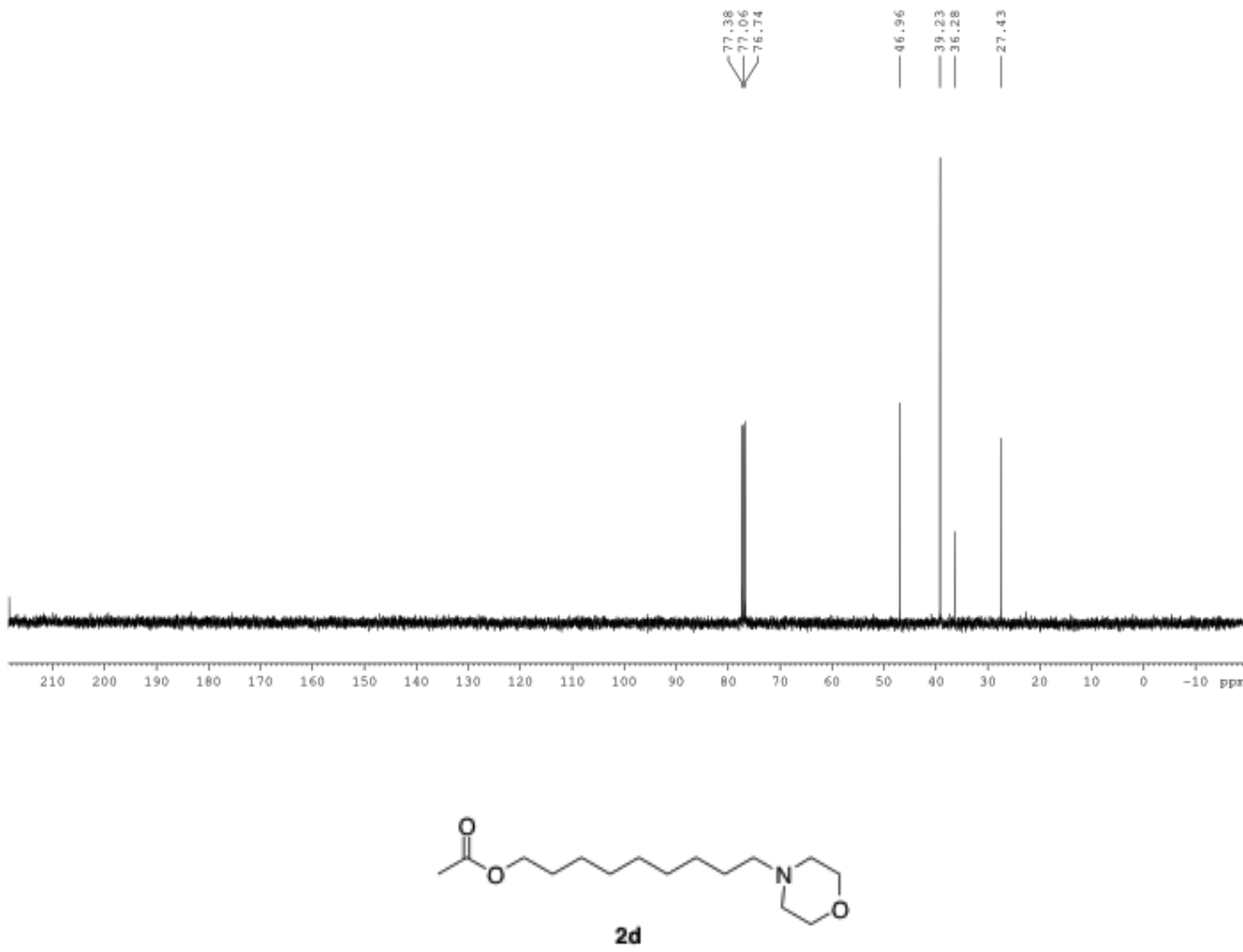
1D Proton NMR ksk01-49
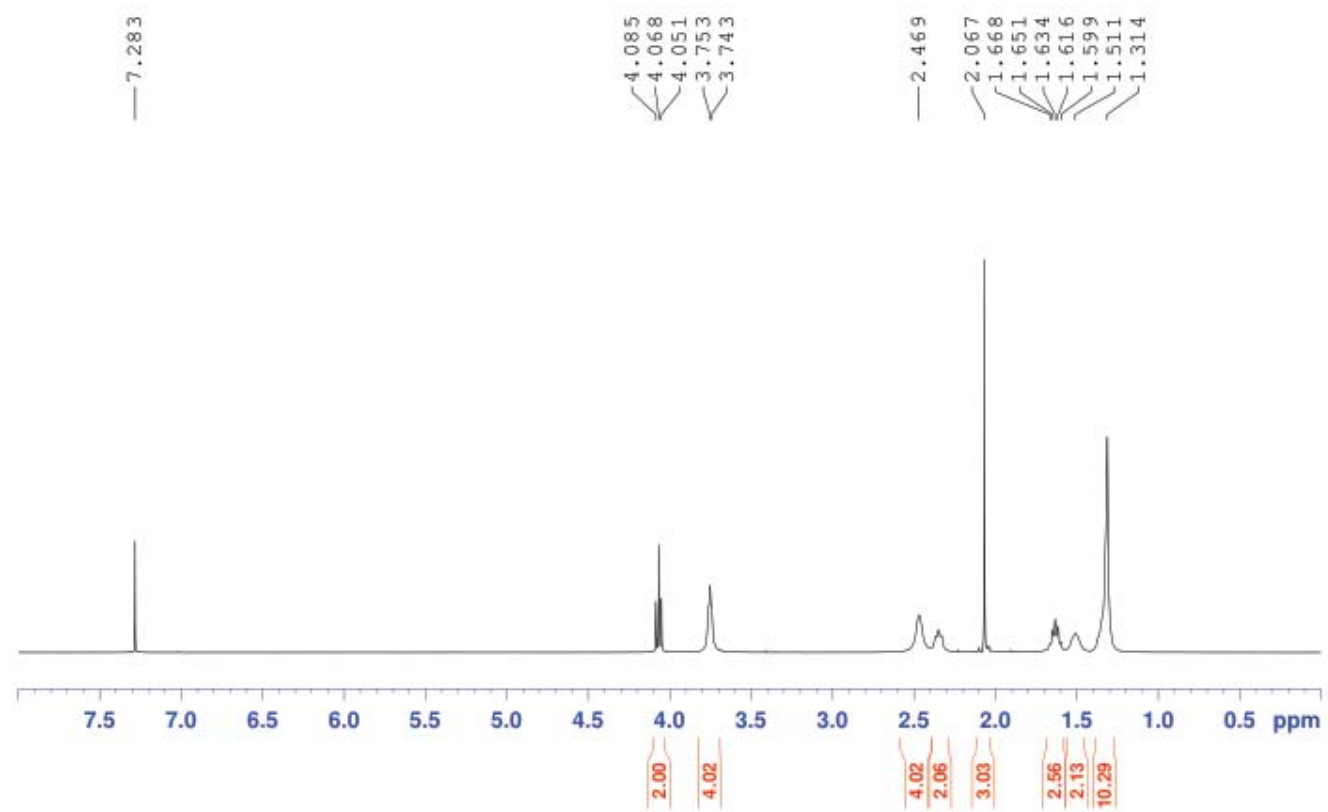

$13 \mathrm{C}$

ksk01-49

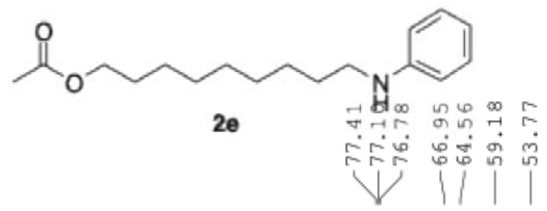

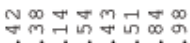

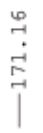

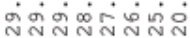

4

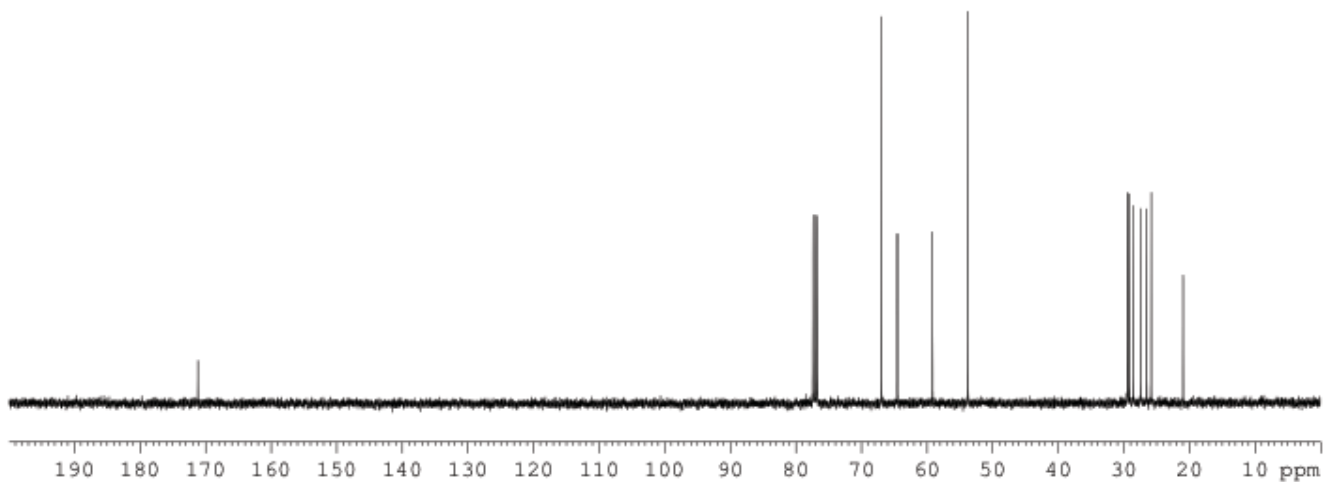


1D Proton NMR
ksk01-82

ksk01-82

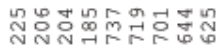

نripipi

귁용

نंग

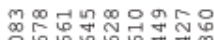

ifinifini

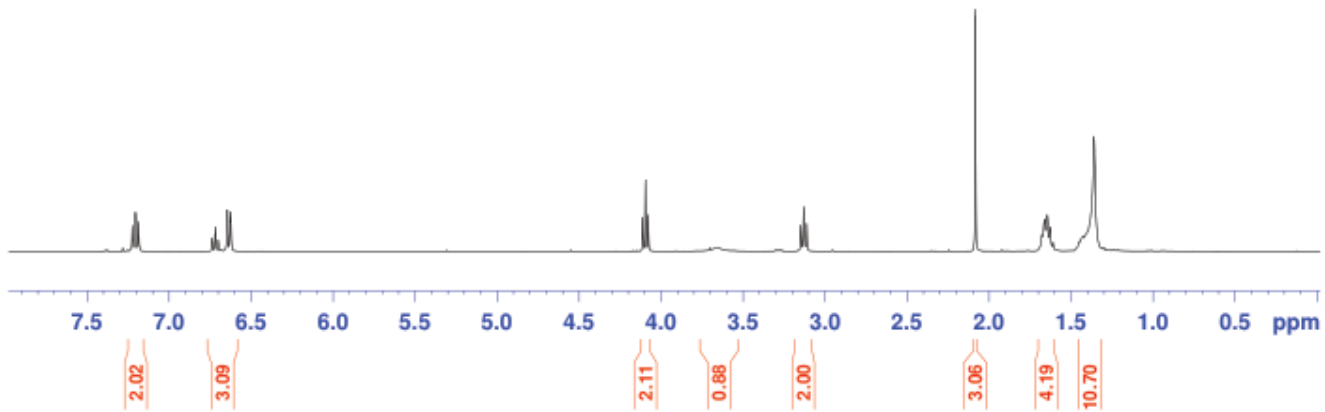

$13 \mathrm{C}$

ksk01-82
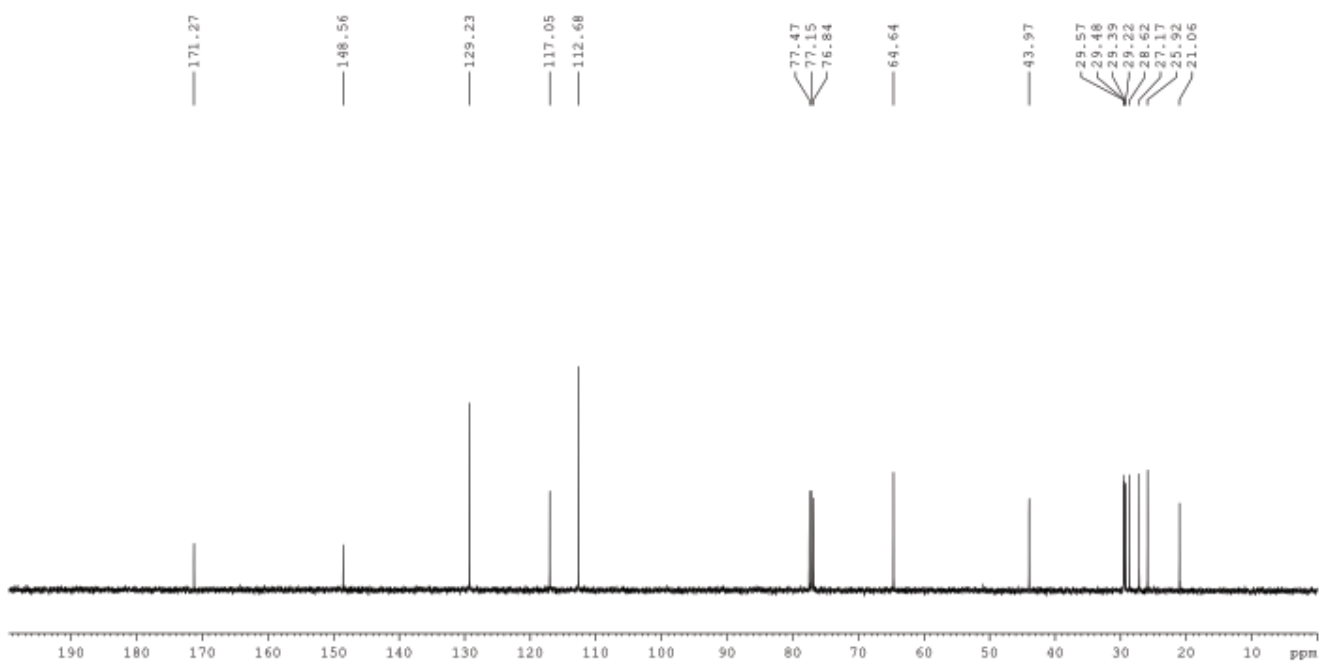


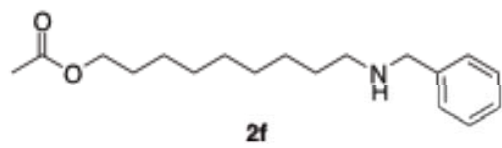

1D Proton NMR

$2 f$

ksk01-32 f2
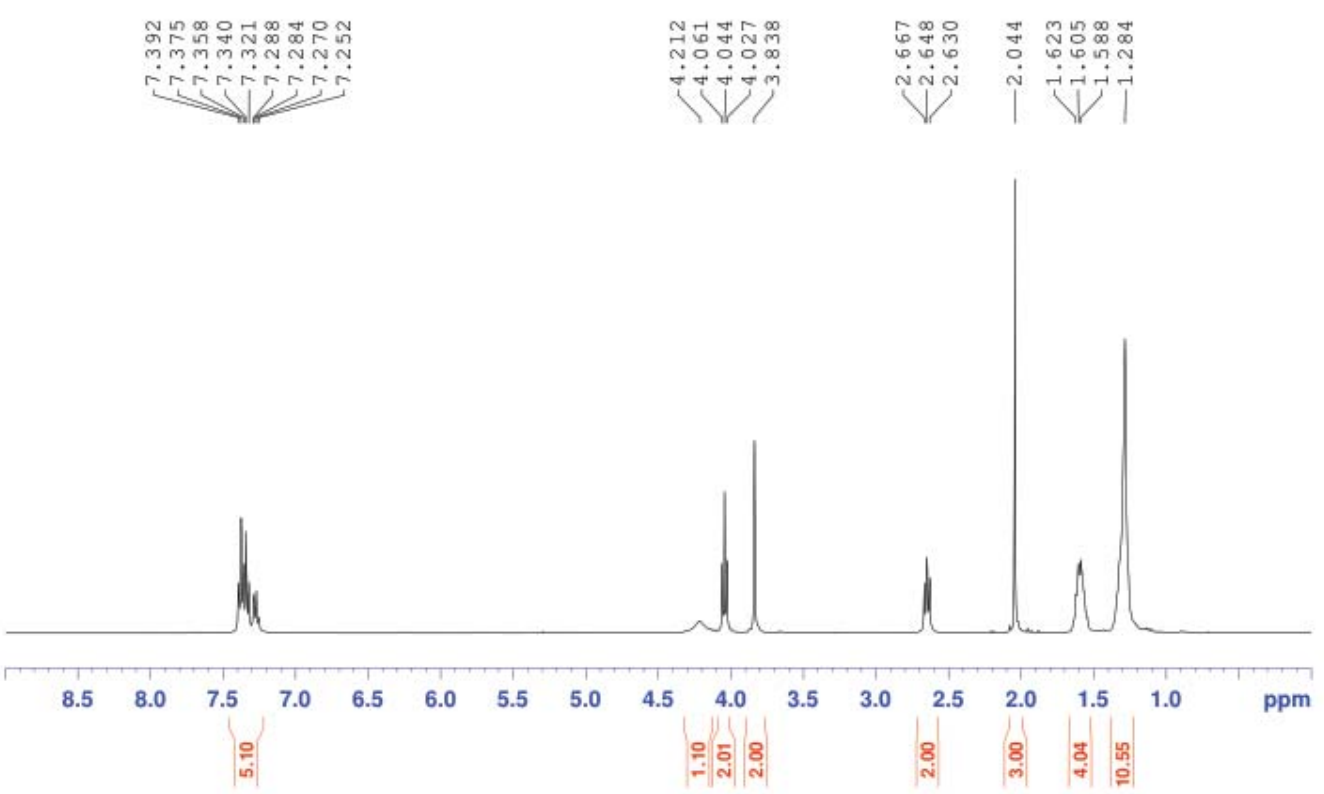

$13 \mathrm{C}$

ksk01-32 f2
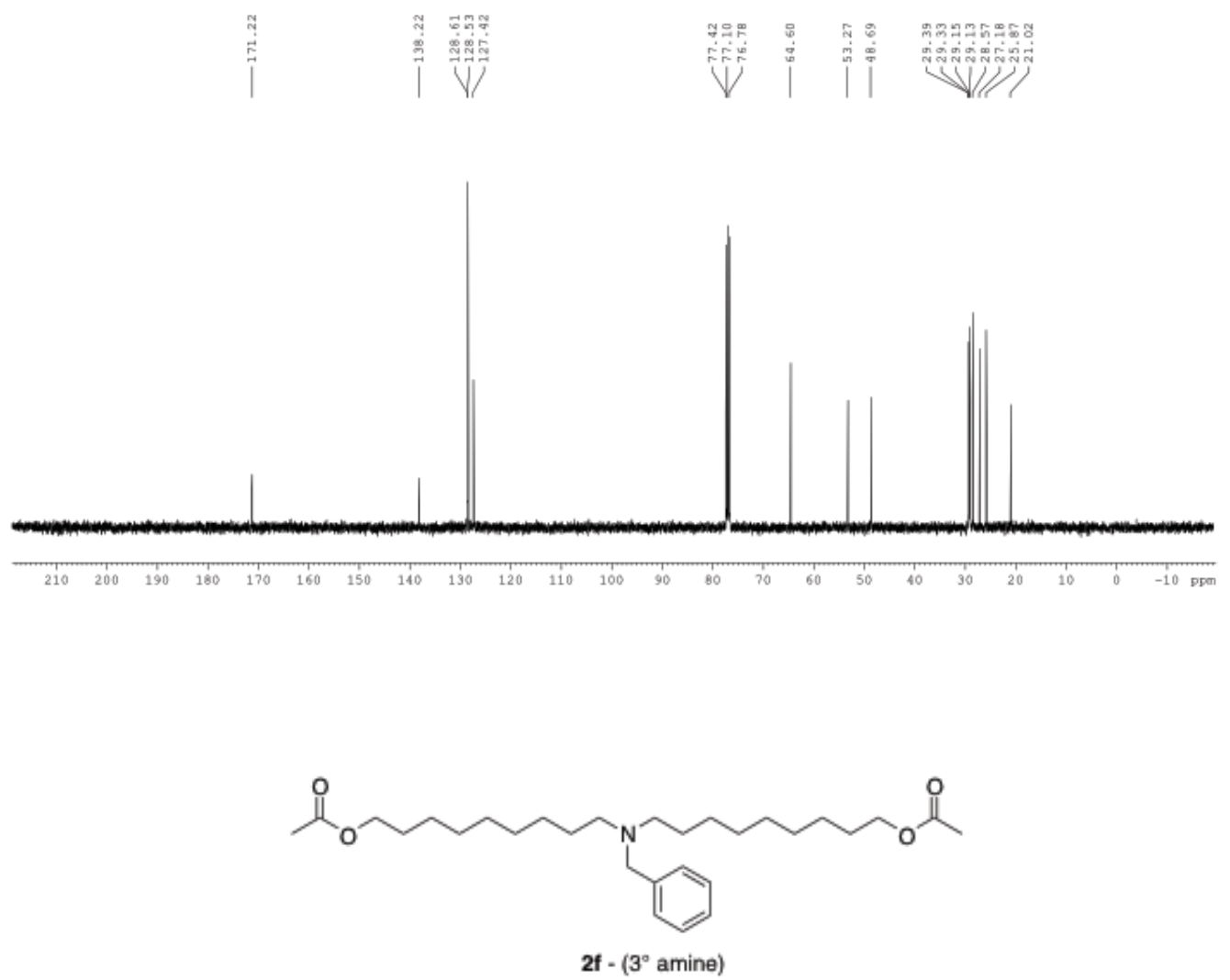

16 

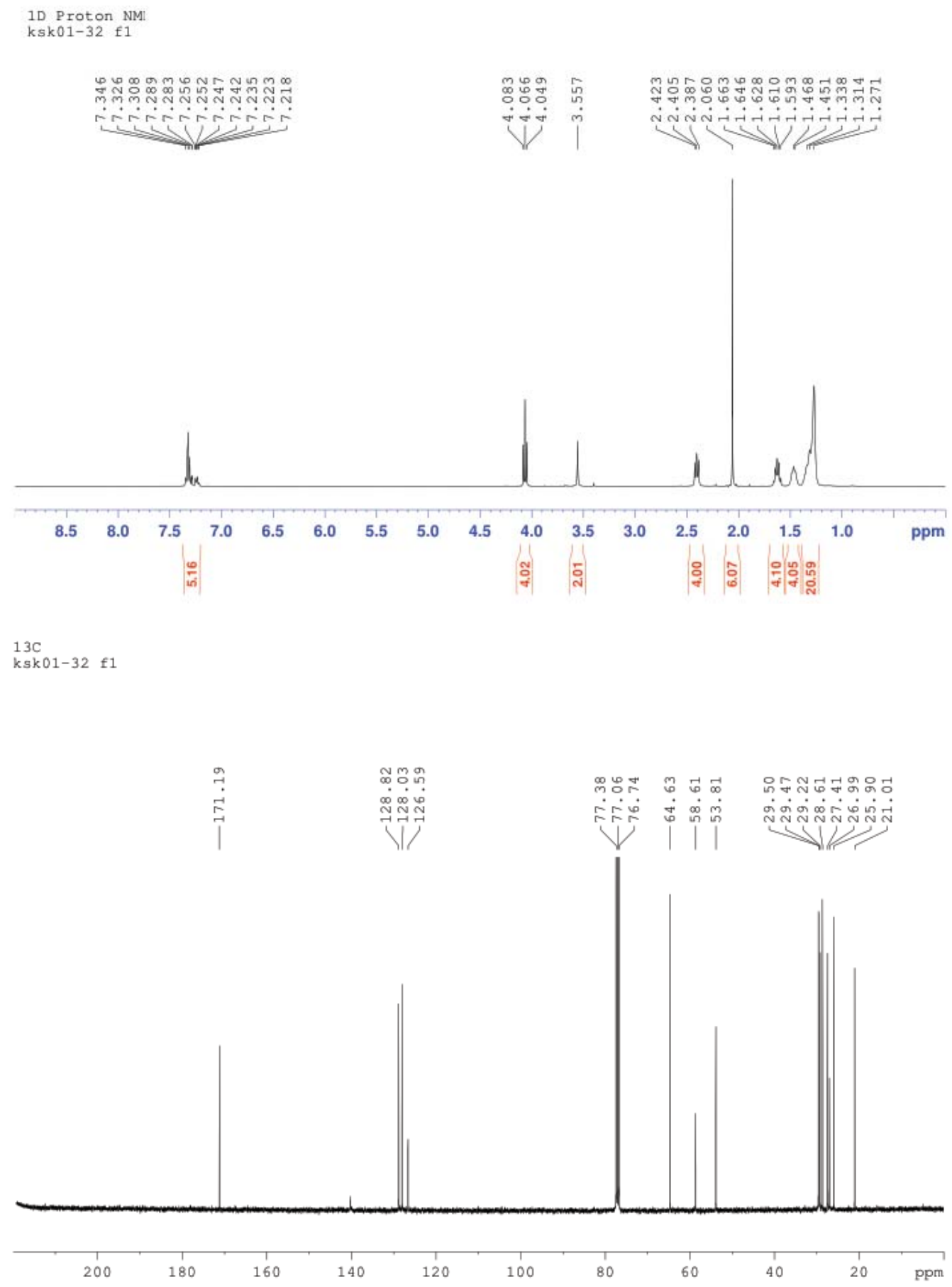


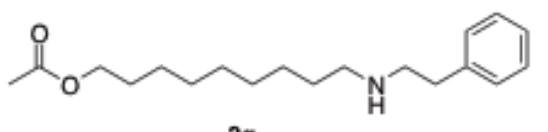

$2 g$

1D Proton NMR

ksk01-90

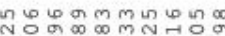

m.

risingrising

5:⿱

गं vinininininjiji

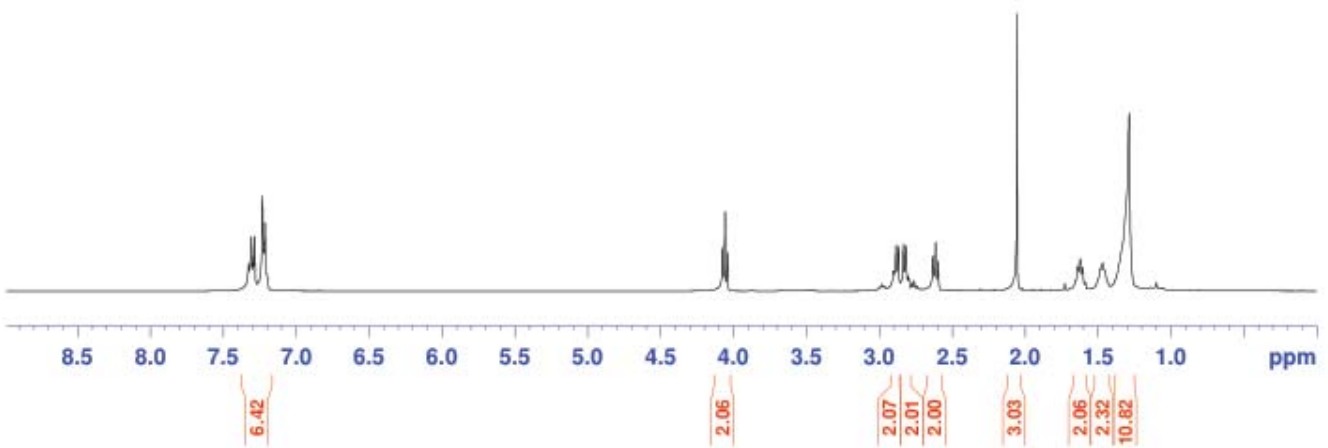

$13 \mathrm{C}$

ksk01-90
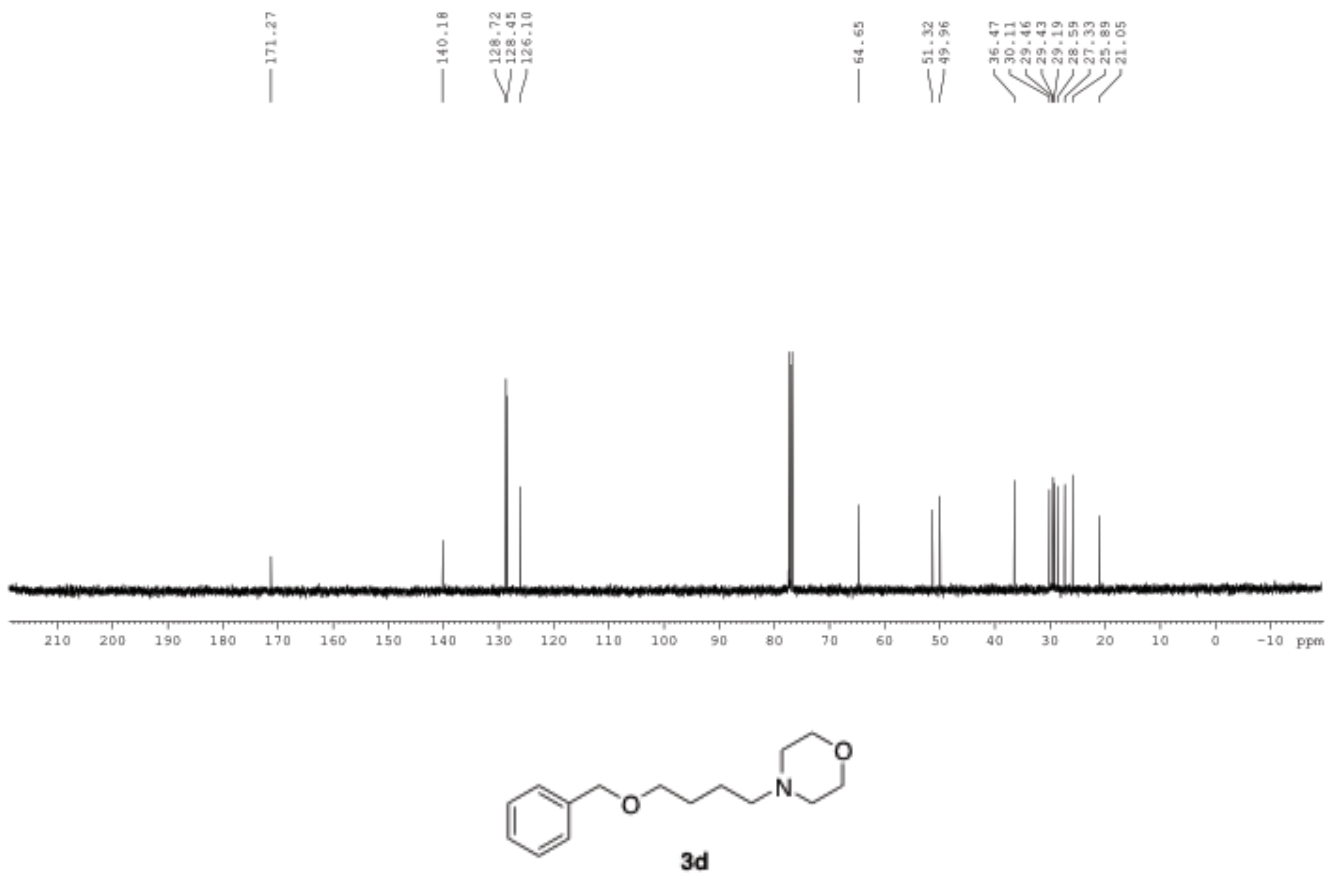
1D Proton NMR

ksk01-71

$400 \mathrm{MHz}, \mathrm{CDCl} 3$
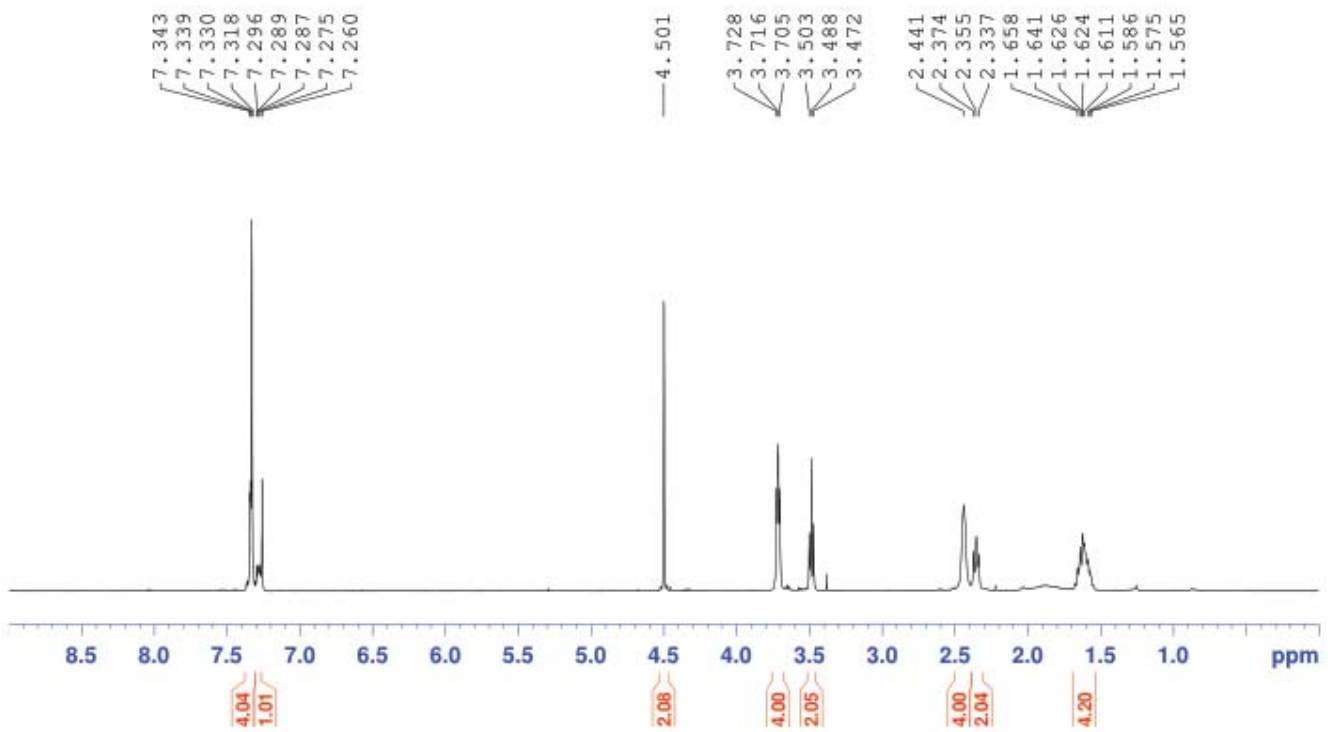

130

ksk01-71
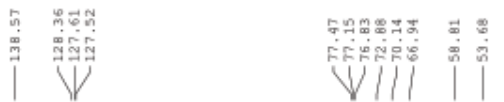

|
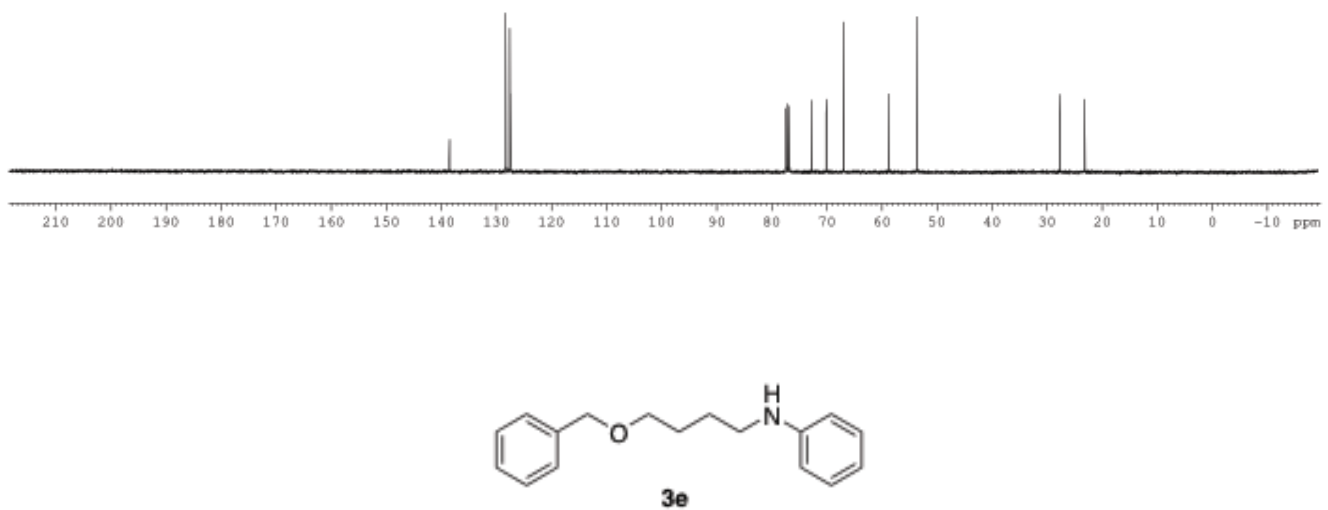


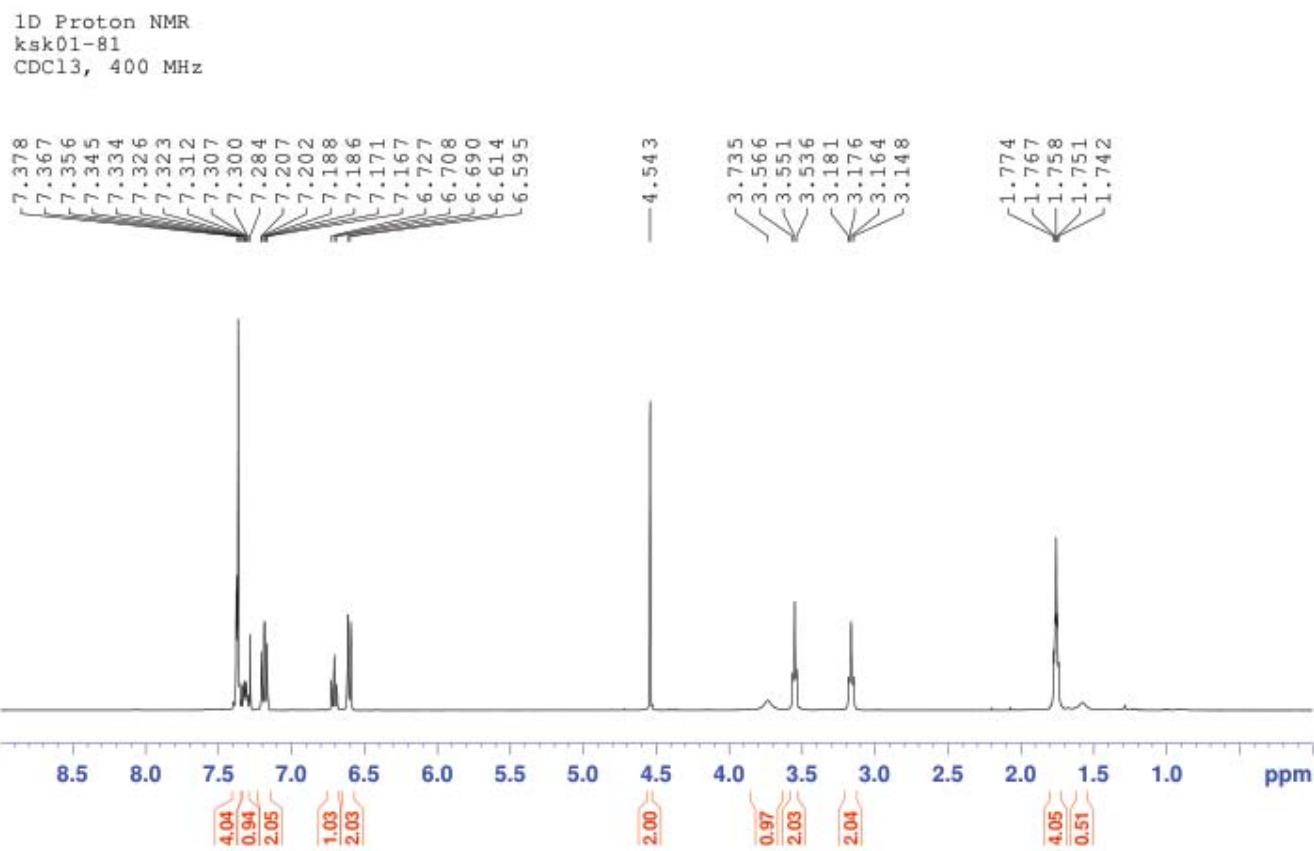

$13 \mathrm{C} \mathrm{ksk01-81}$

CDC13
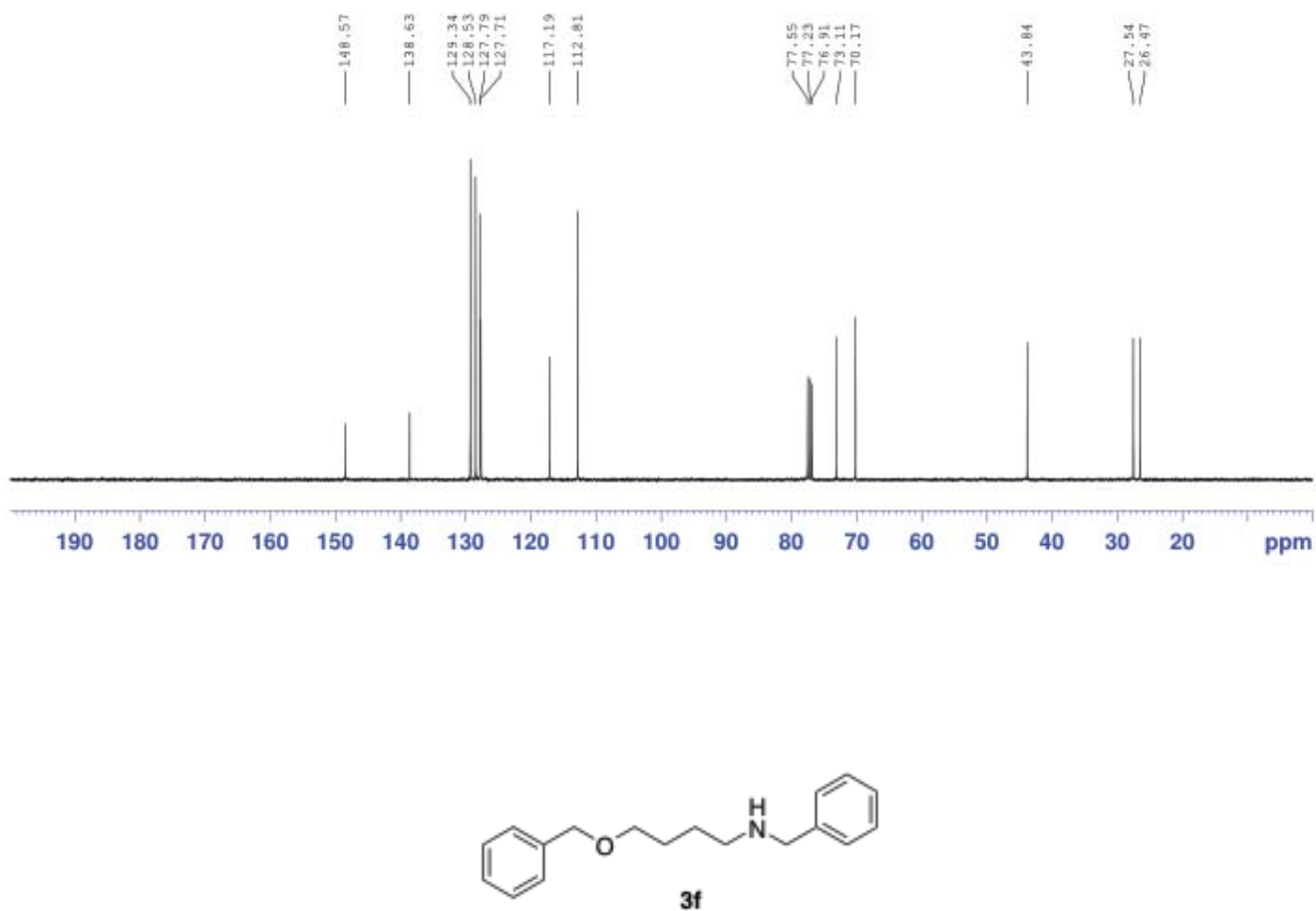
1D Proton NMR

ksk01-83

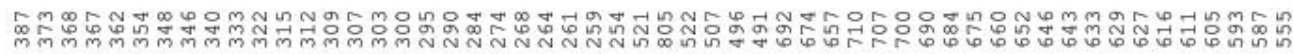

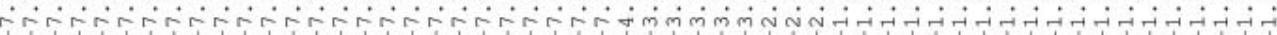

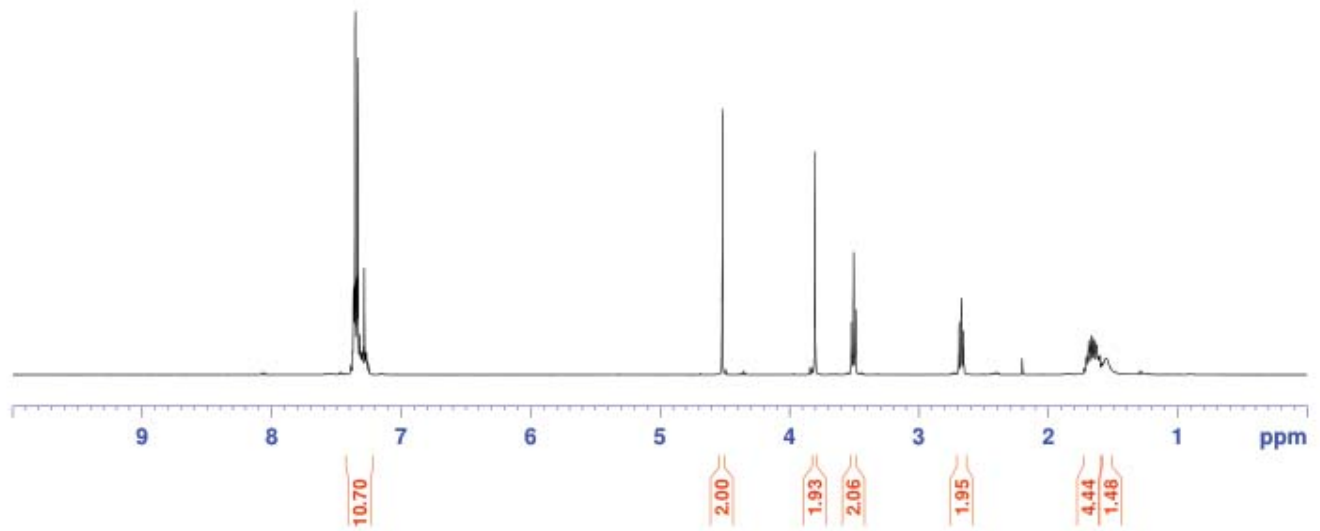

ksk01-83
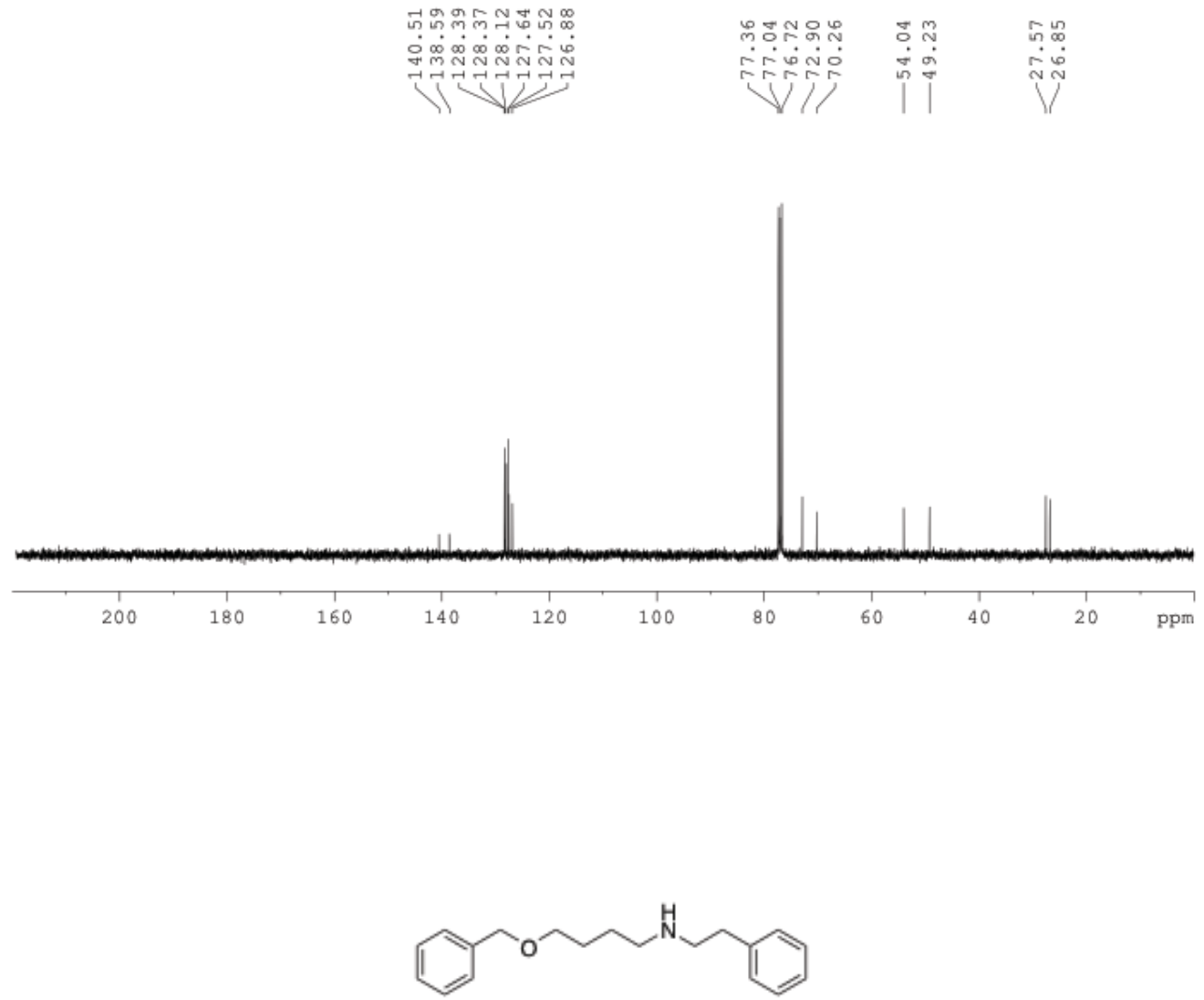

$3 g$ 


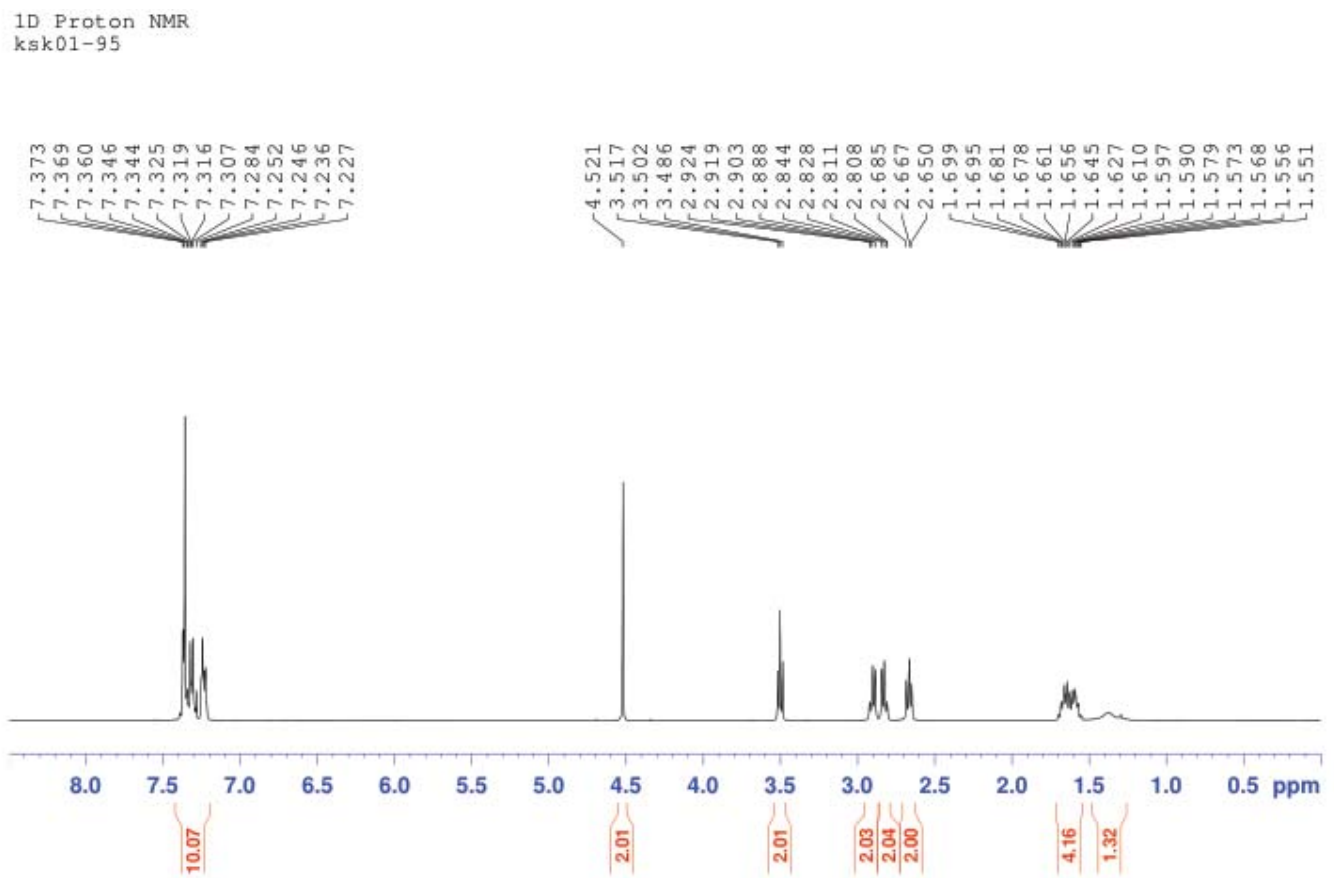

$13 \mathrm{C}$

ksk01-95

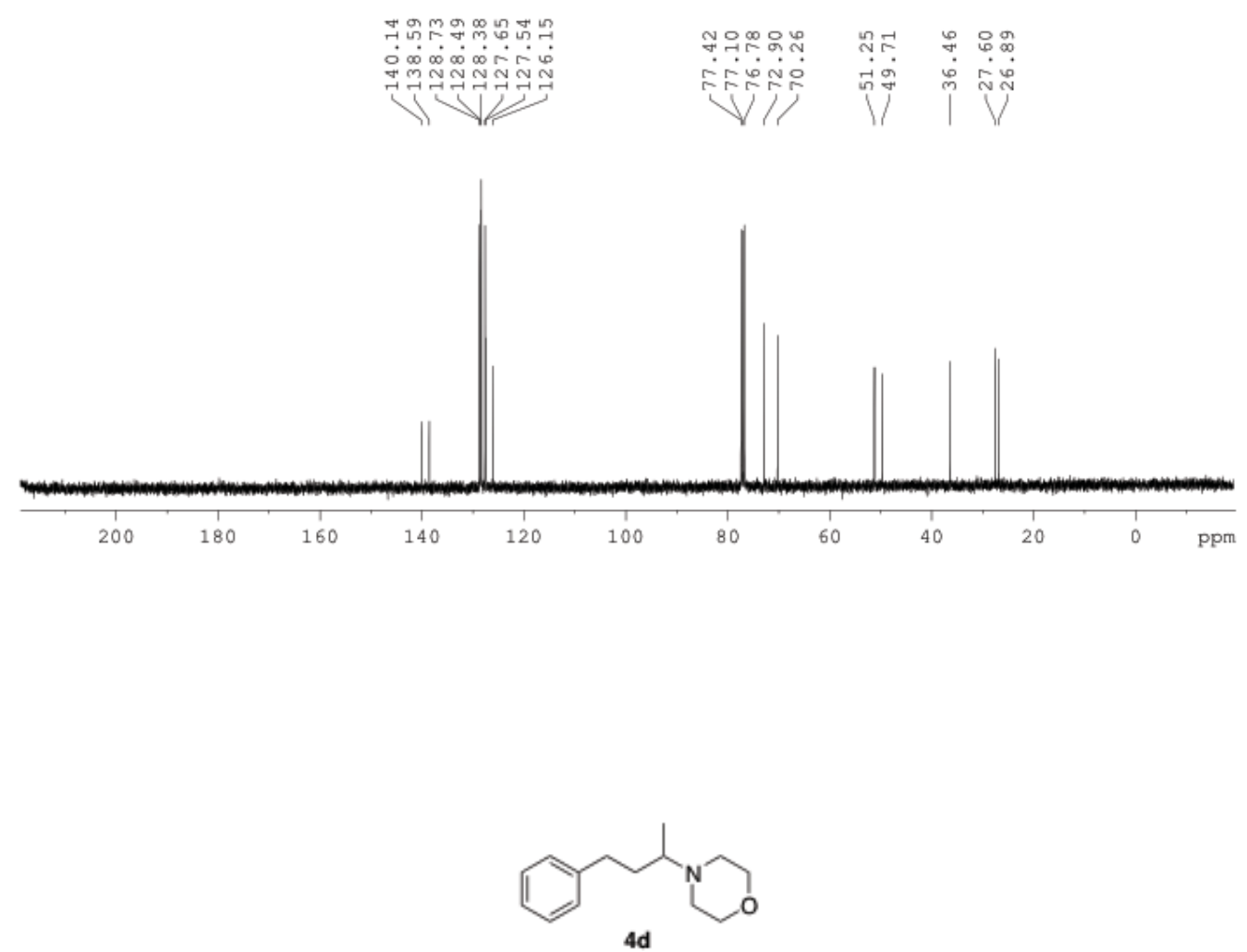


1D Proton NMR

ksk01-86

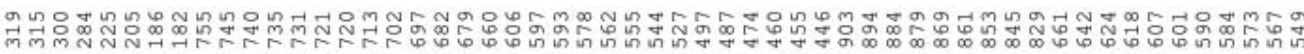

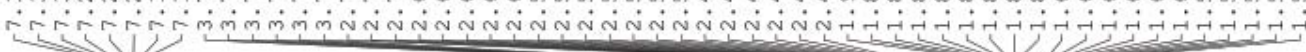

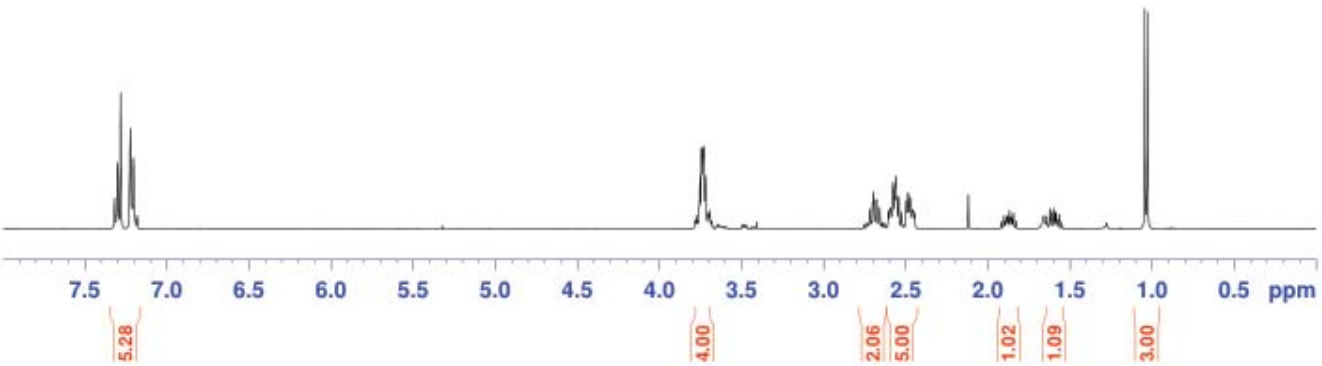

$13 \mathrm{C}$

ksk01-86
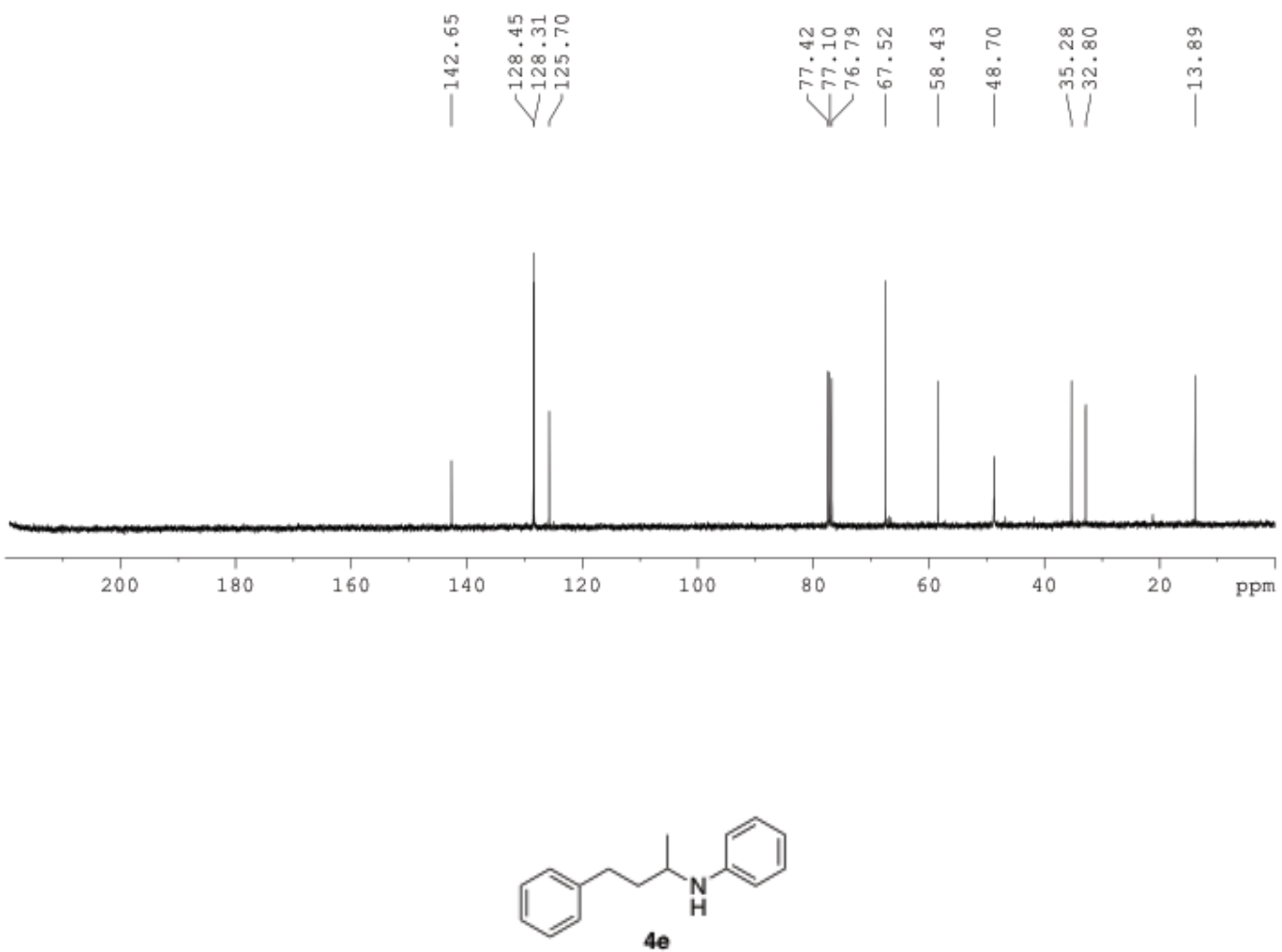


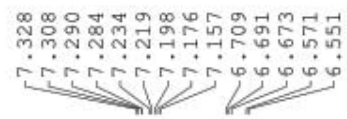

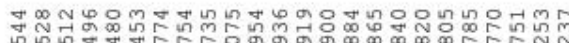


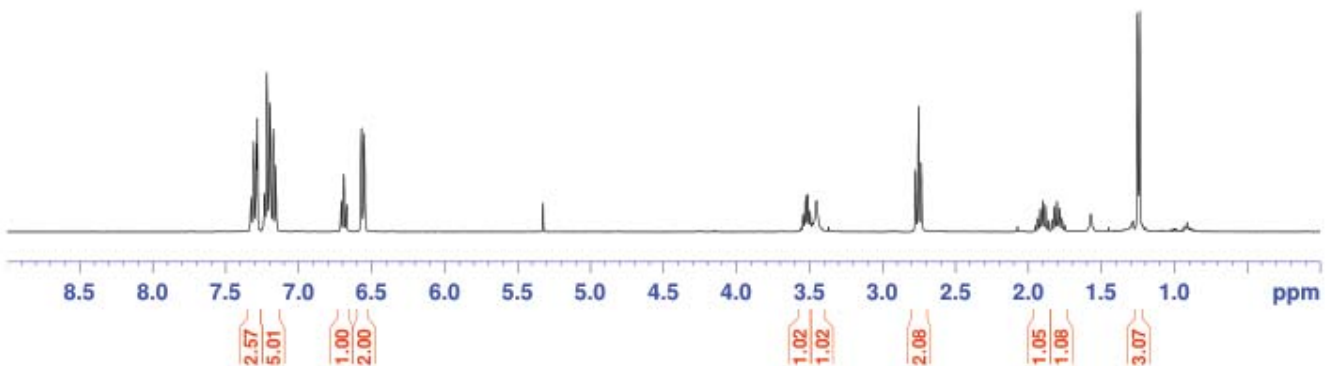

$13 \mathrm{C}$

ksk01-87
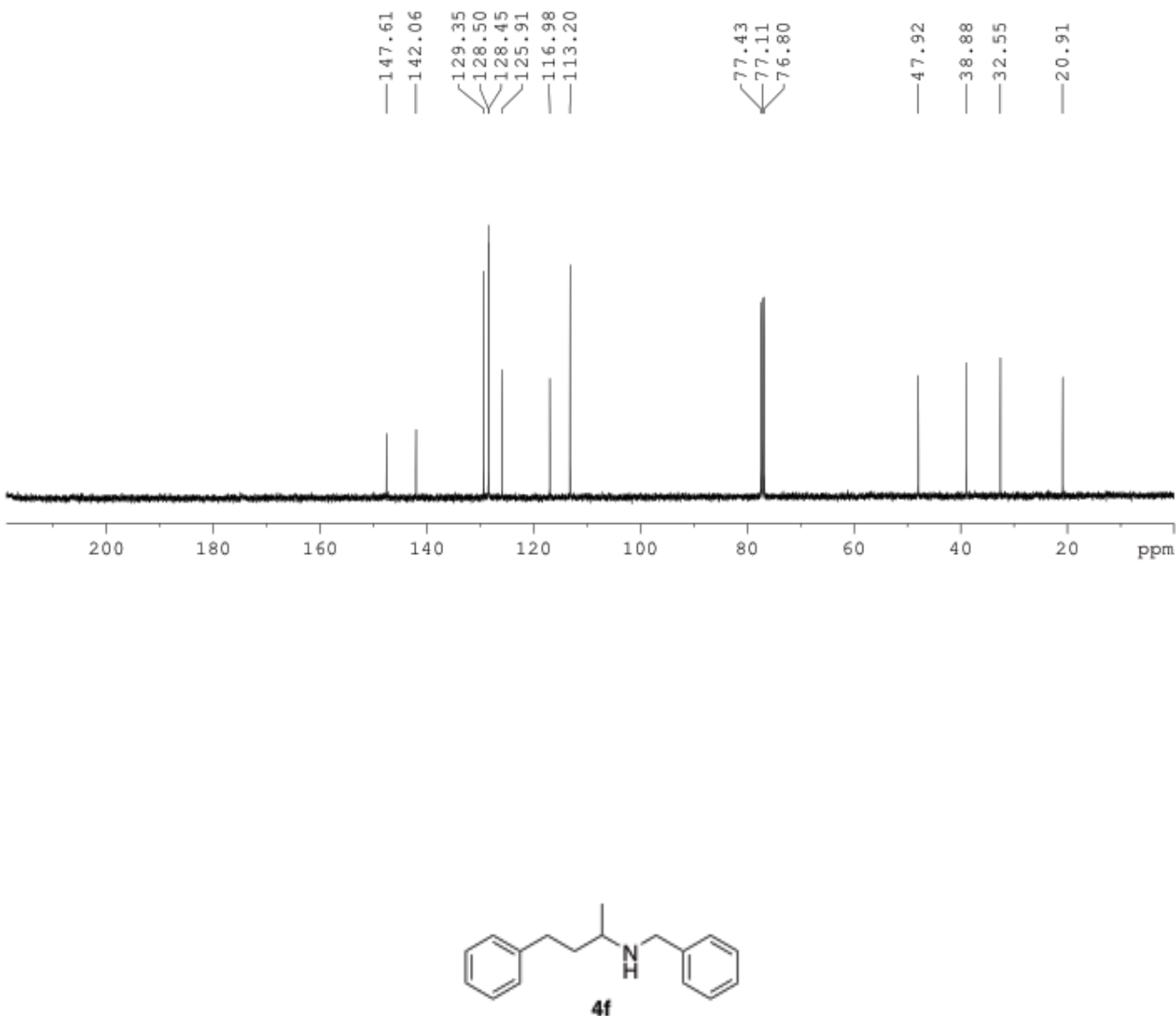


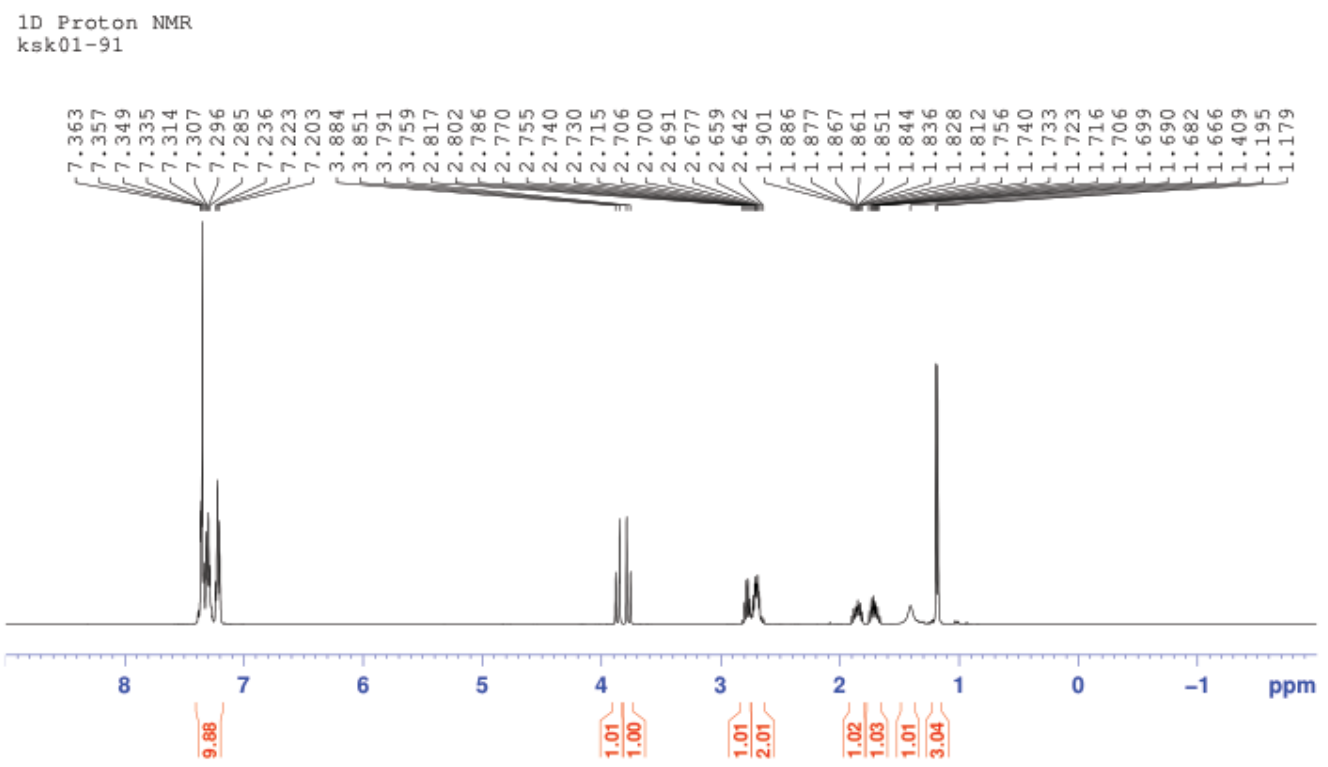

$13 \mathrm{C}$

$13 \mathrm{C}$
ksk01-91
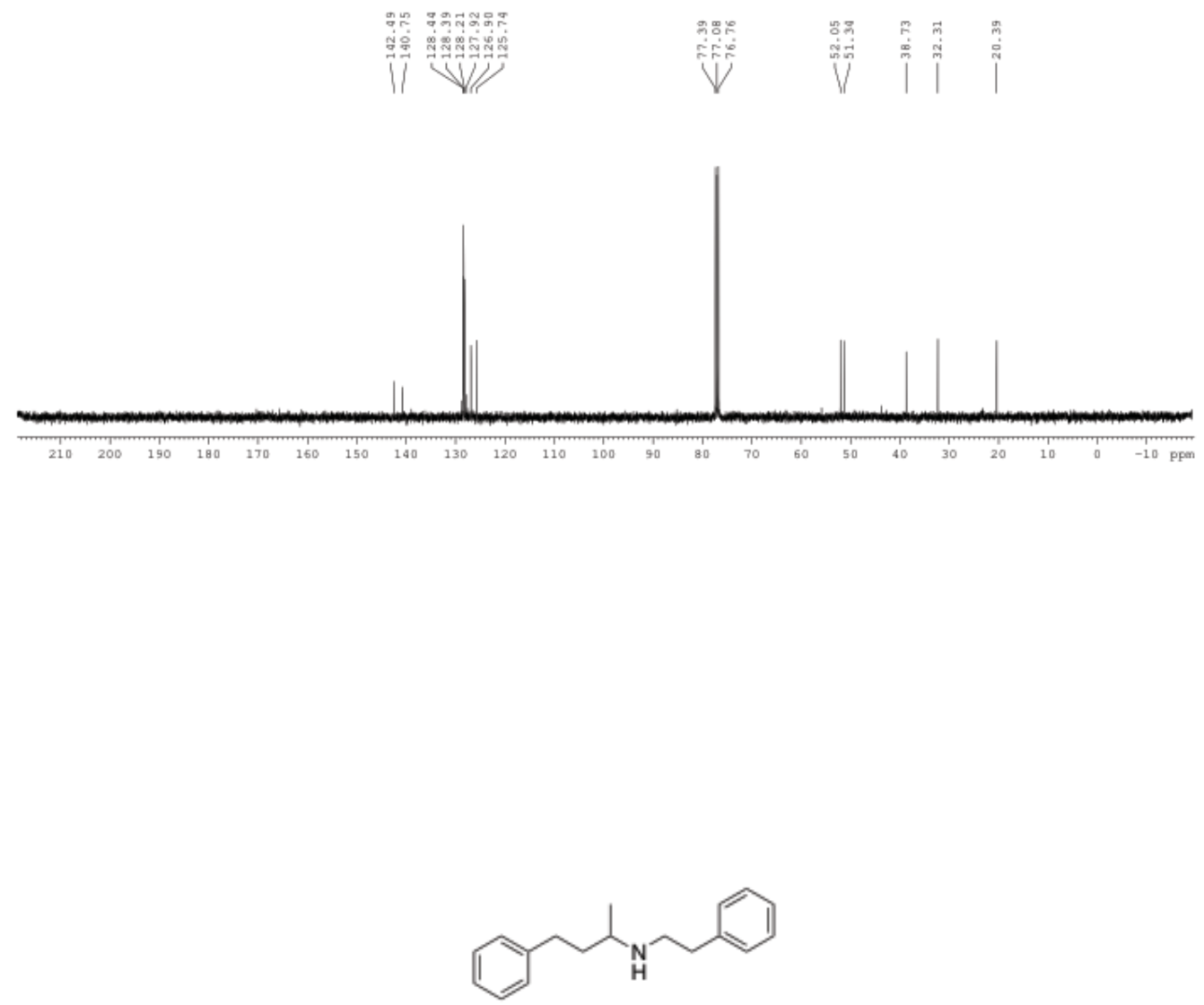

$4 g$ 
1D Proton NMR

ksk01-96

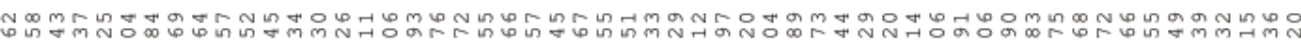

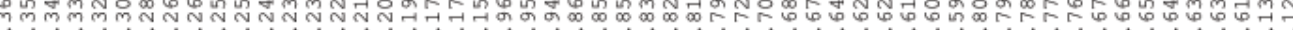

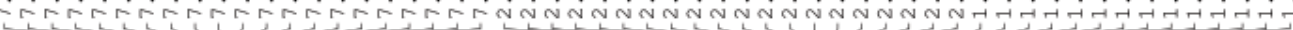

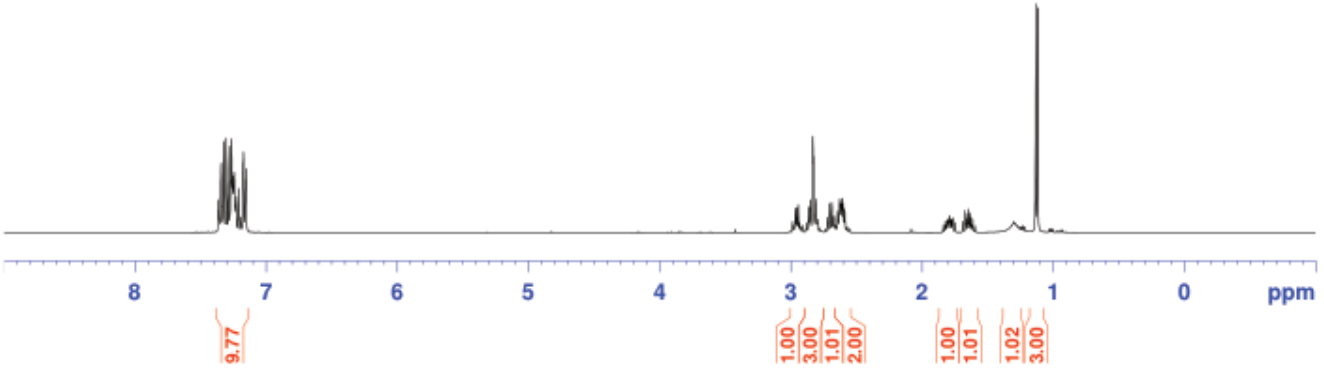

$13 \mathrm{C}$

ksk01-96

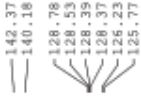

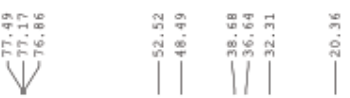

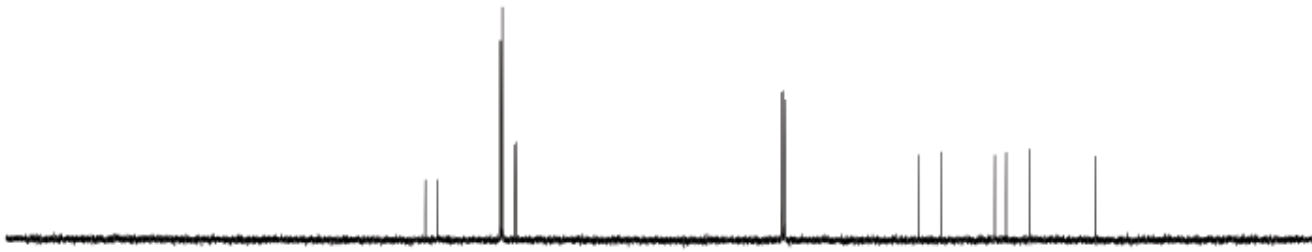

$\begin{array}{llllllllllllllllllllllllllllll}210 & 200 & 190 & 180 & 170 & 160 & 150 & 140 & 130 & 120 & 110 & 100 & 90 & 80 & 70 & 60 & 50 & 40 & 30 & 20 & 10 & 0 & -10 & \text { Dpn }\end{array}$

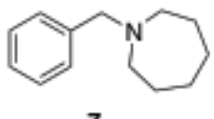


1D Proton NMR

ksk02-63

RRYKER

年

cririris

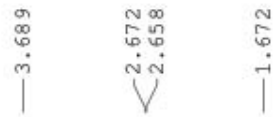

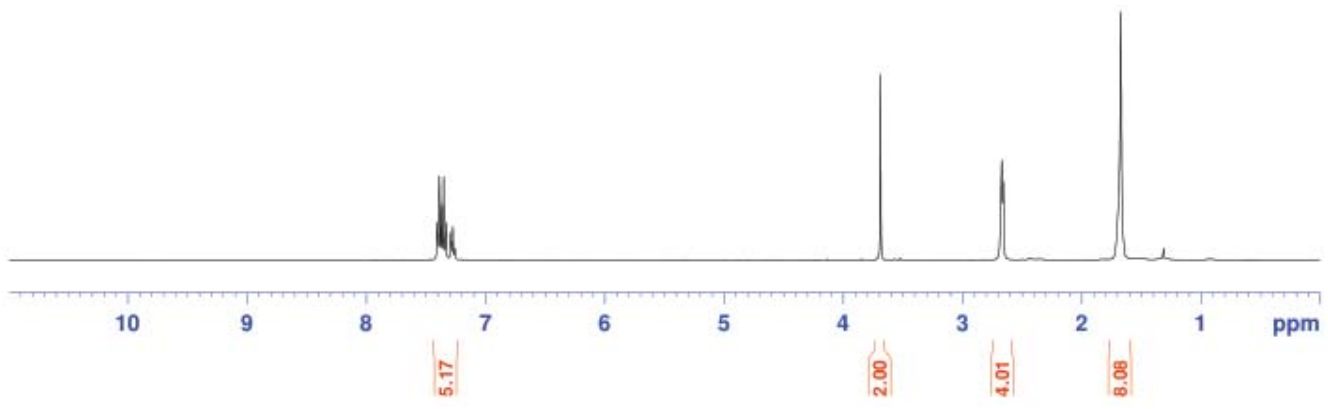

ksk02-63

$400 \mathrm{MHz}, \mathrm{CDCl} 3$
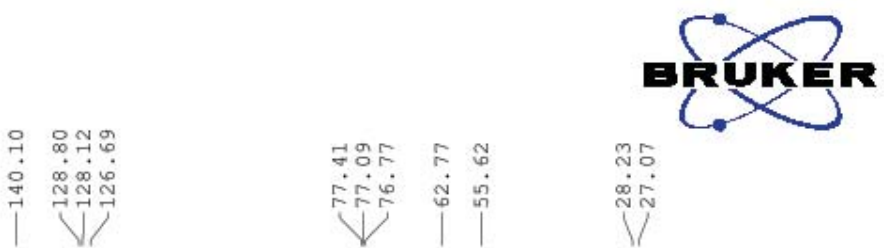

구ㅇㅛㅛ 응

ํㅗㅇ

mo

㐫
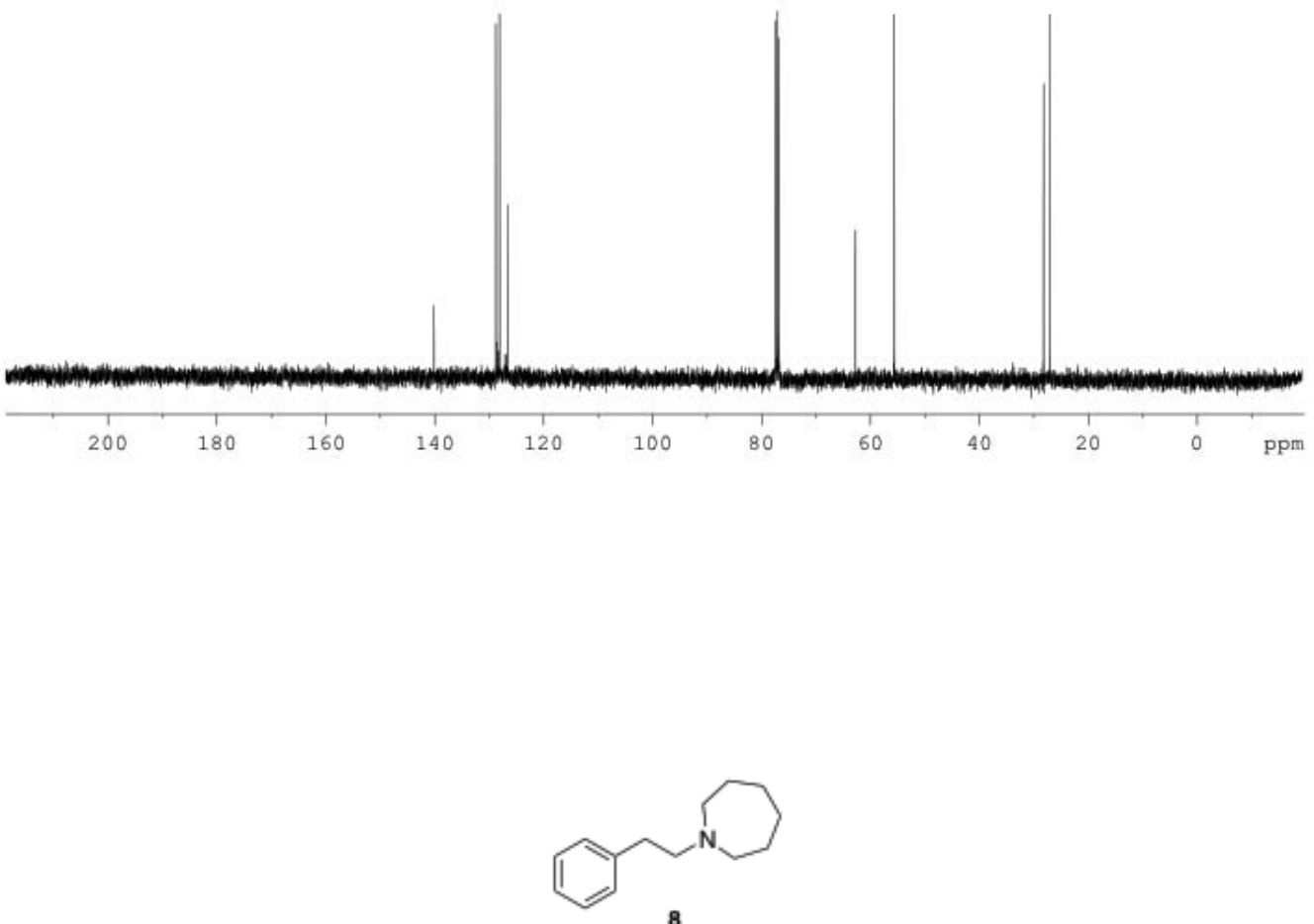
1D Proton NMR

ksk02-64

$400 \mathrm{MHz}, \operatorname{CDCl} 3$

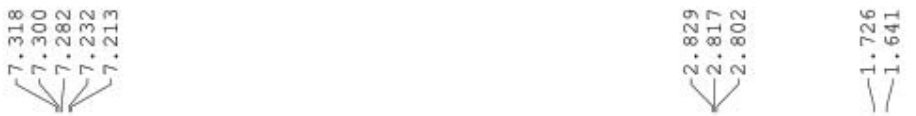

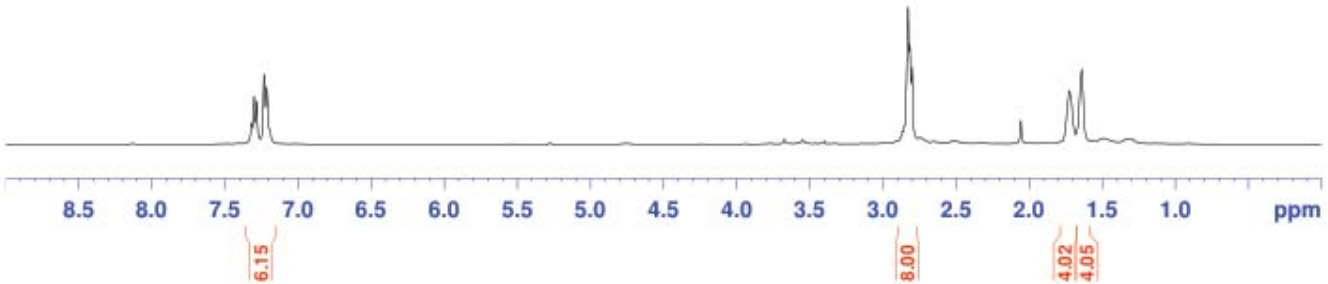

$13 \mathrm{C}$
ksk02-64
$400 \mathrm{MHz}, \quad \mathrm{CDCl} 13$
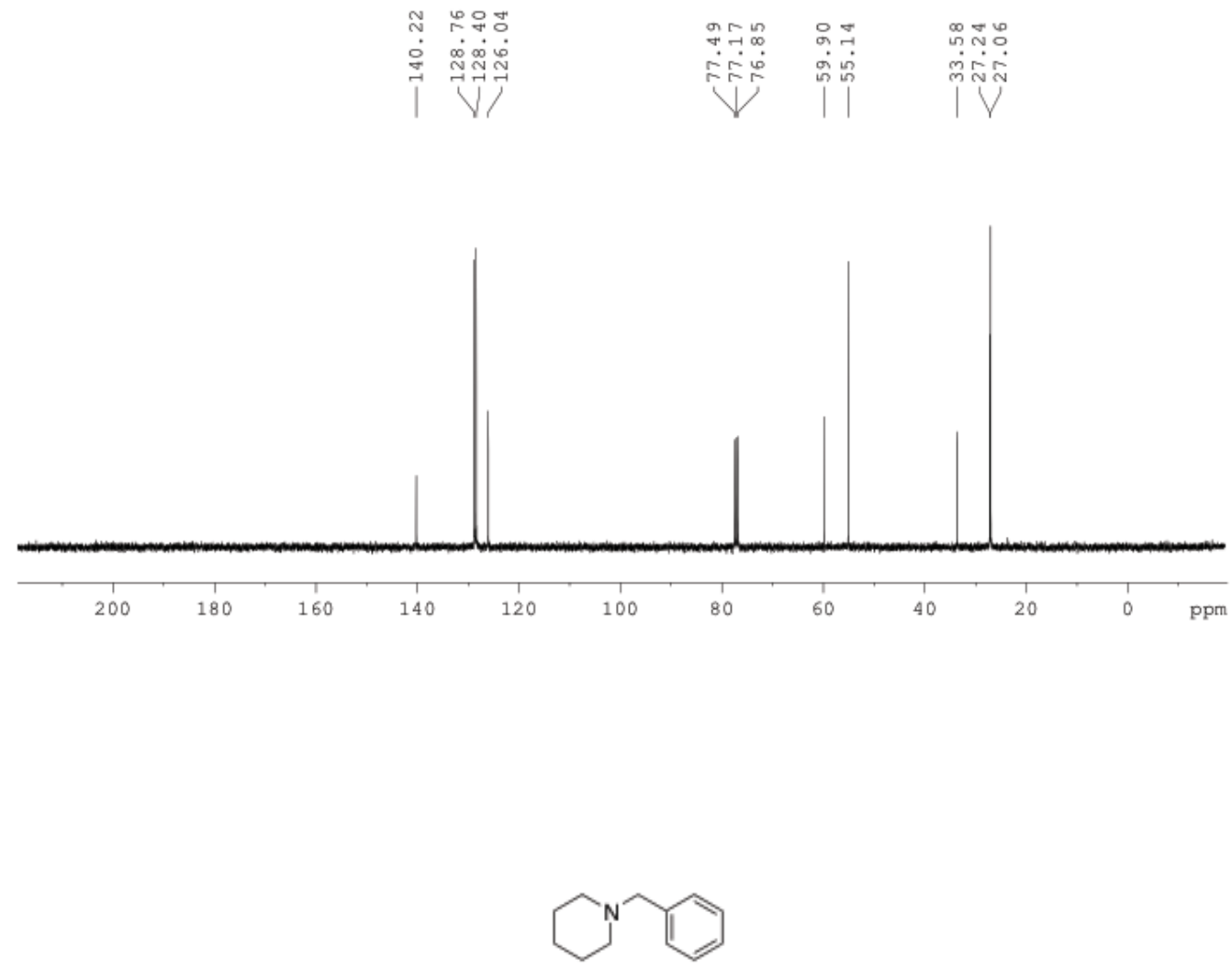

9 


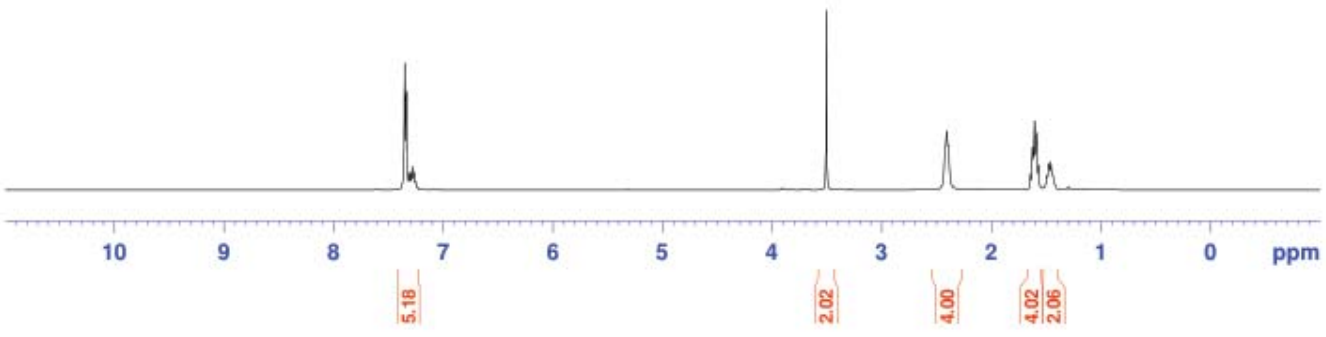

$13 \mathrm{C}$

ksko2-60

$400 \mathrm{MHz}, \mathrm{CDCl} 3$
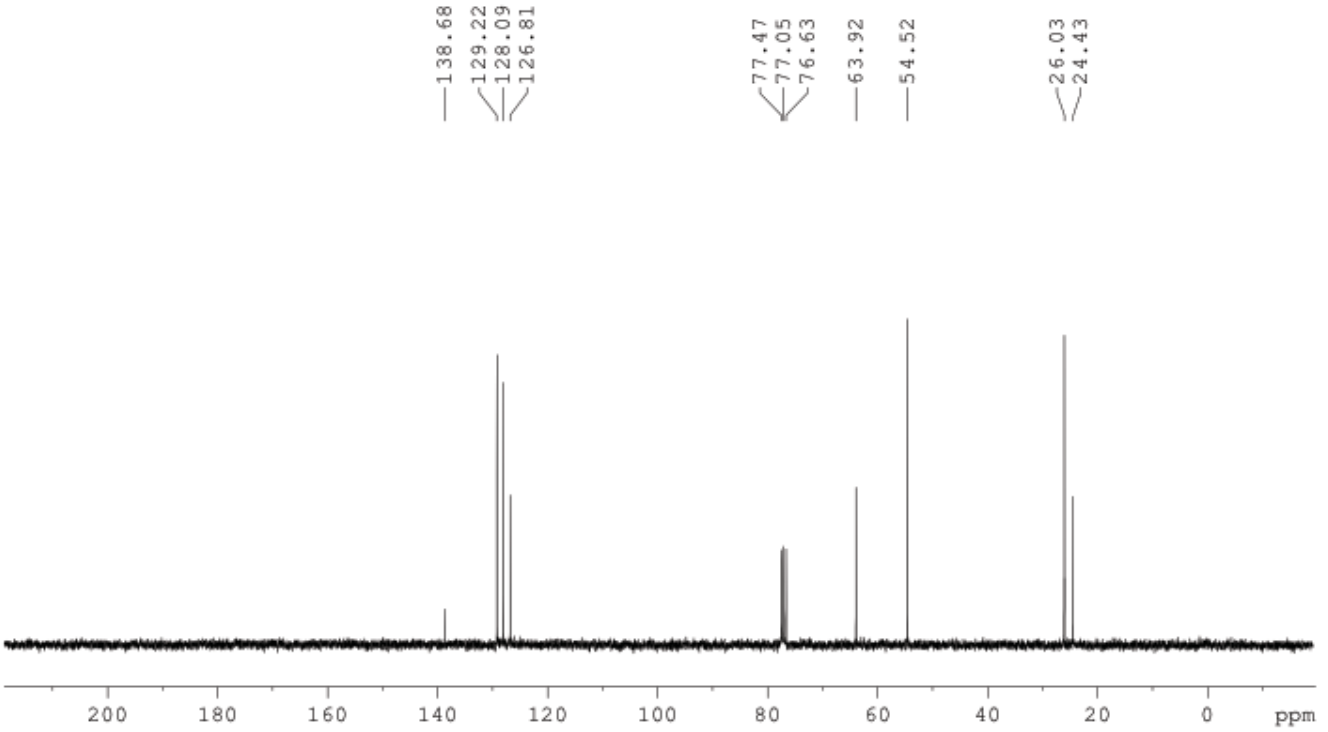


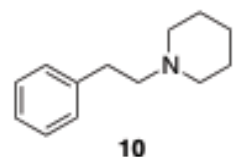

1D Proton NMR ksk01-92

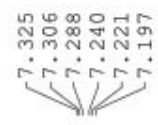

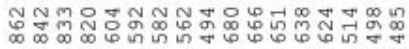

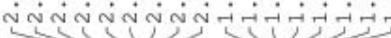
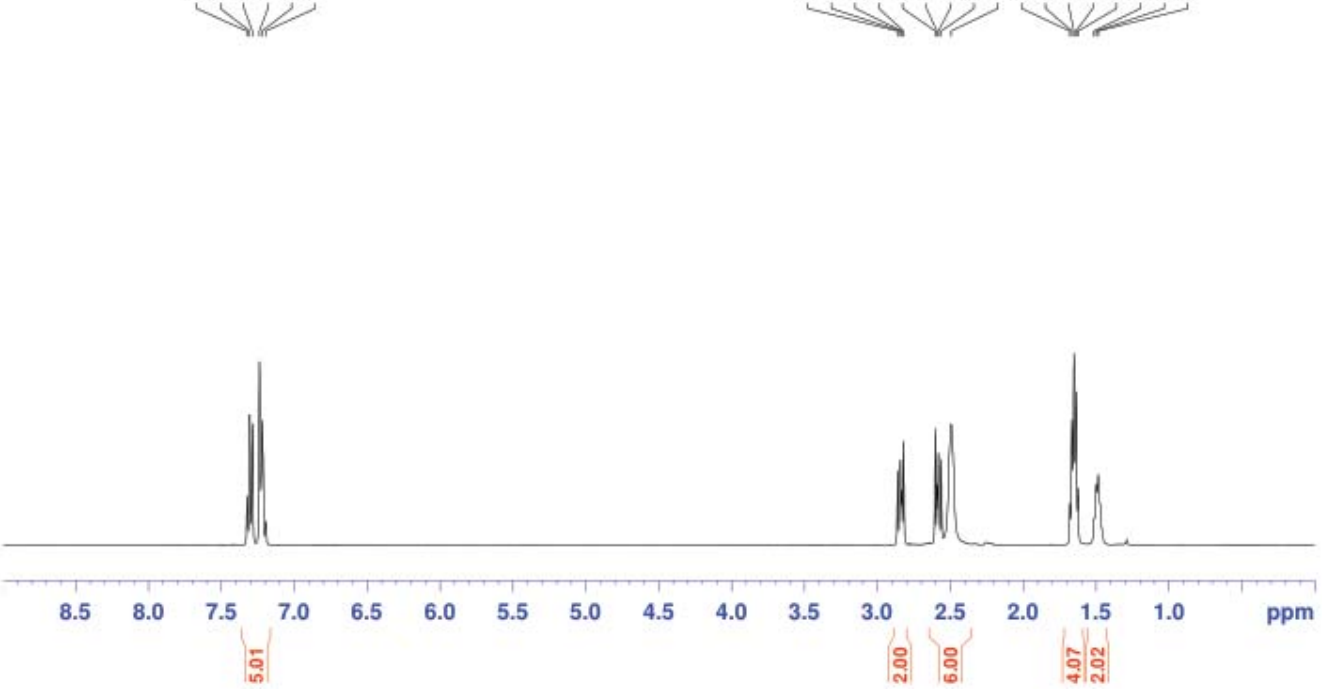

$13 \mathrm{C}$

ksk01-92

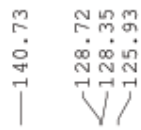

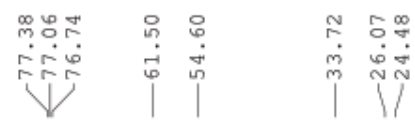
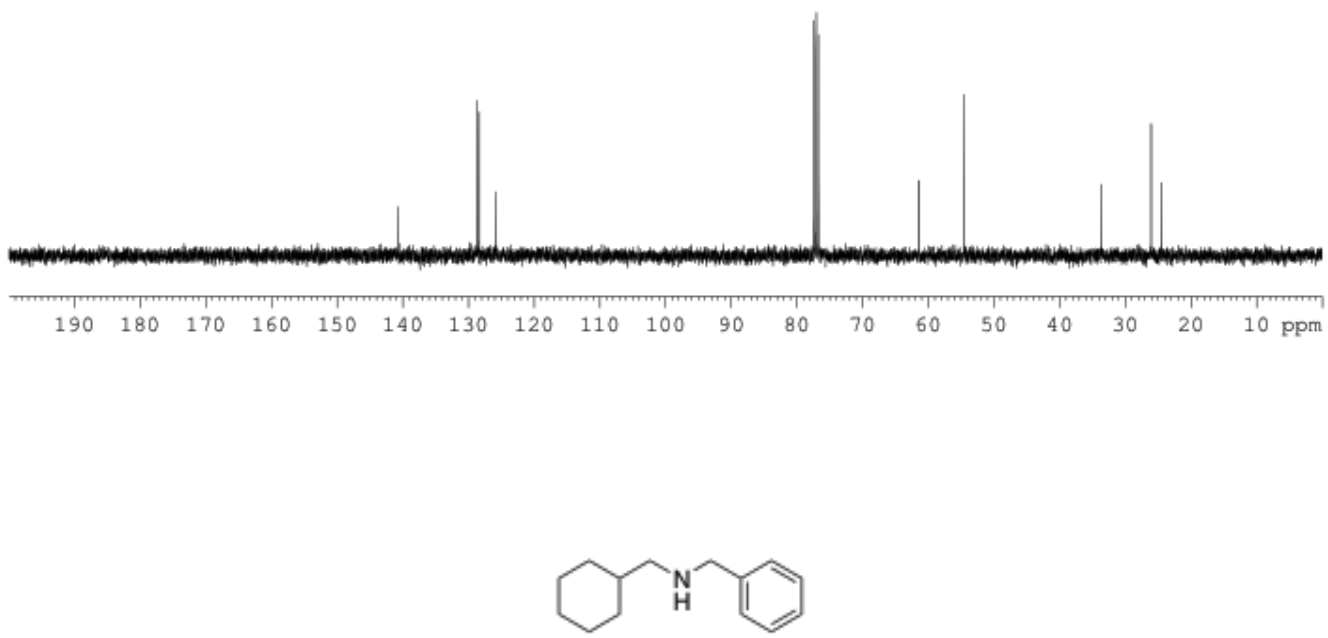

$11 \mathrm{a}$ 

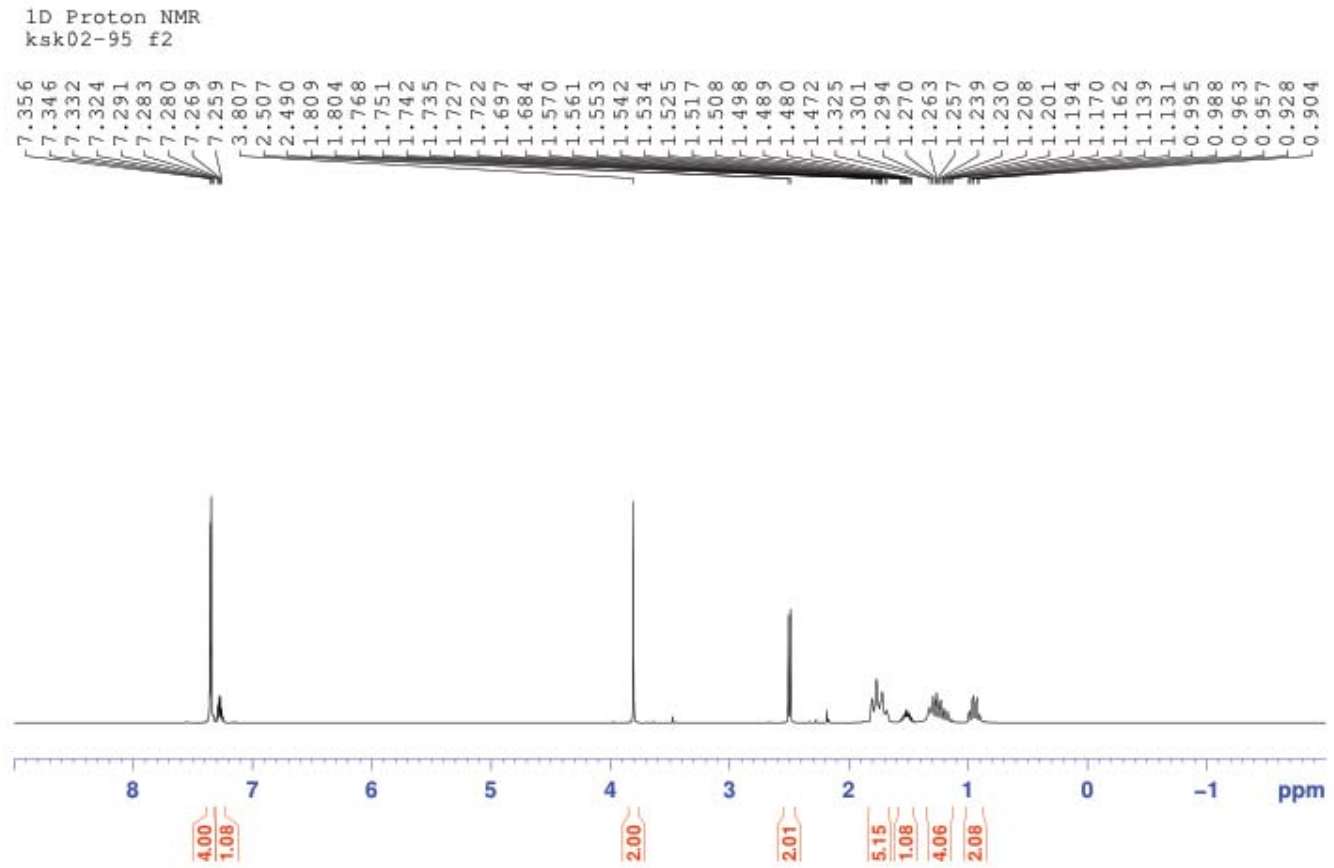

$13 \mathrm{C}$

ksk02-95 f2
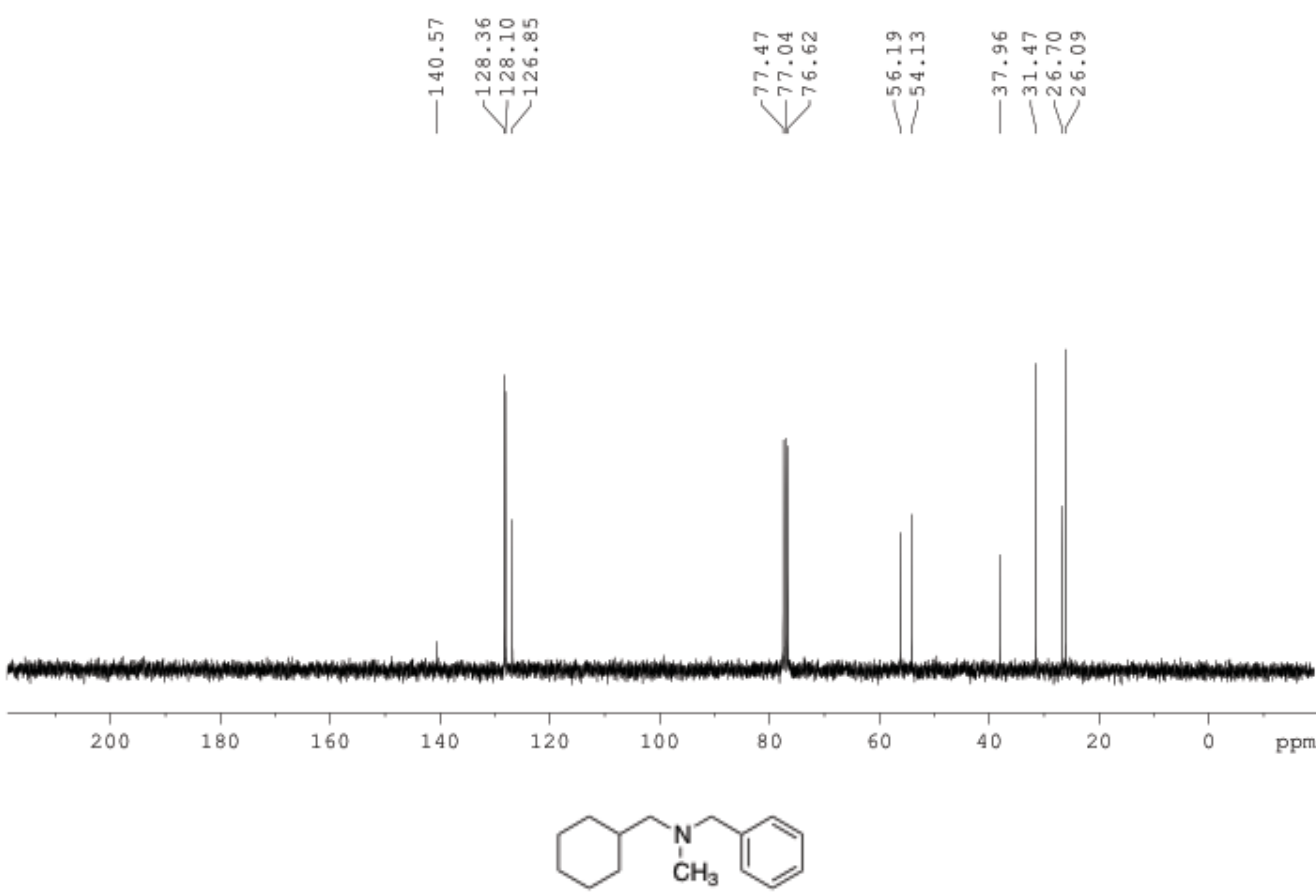

11b 
1D Proton NMR

ksk02-95 f1

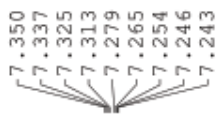

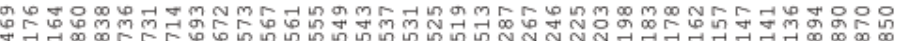

भન

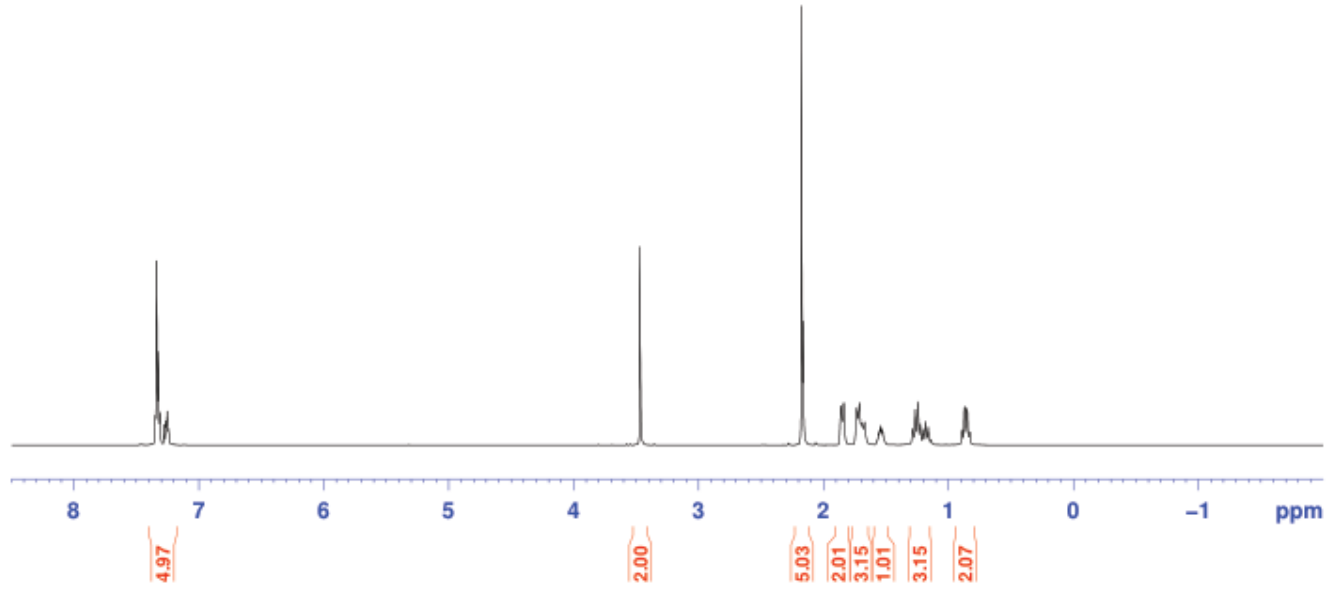

$13 \mathrm{C} \mathrm{NMR}$

ksk02-95 f1
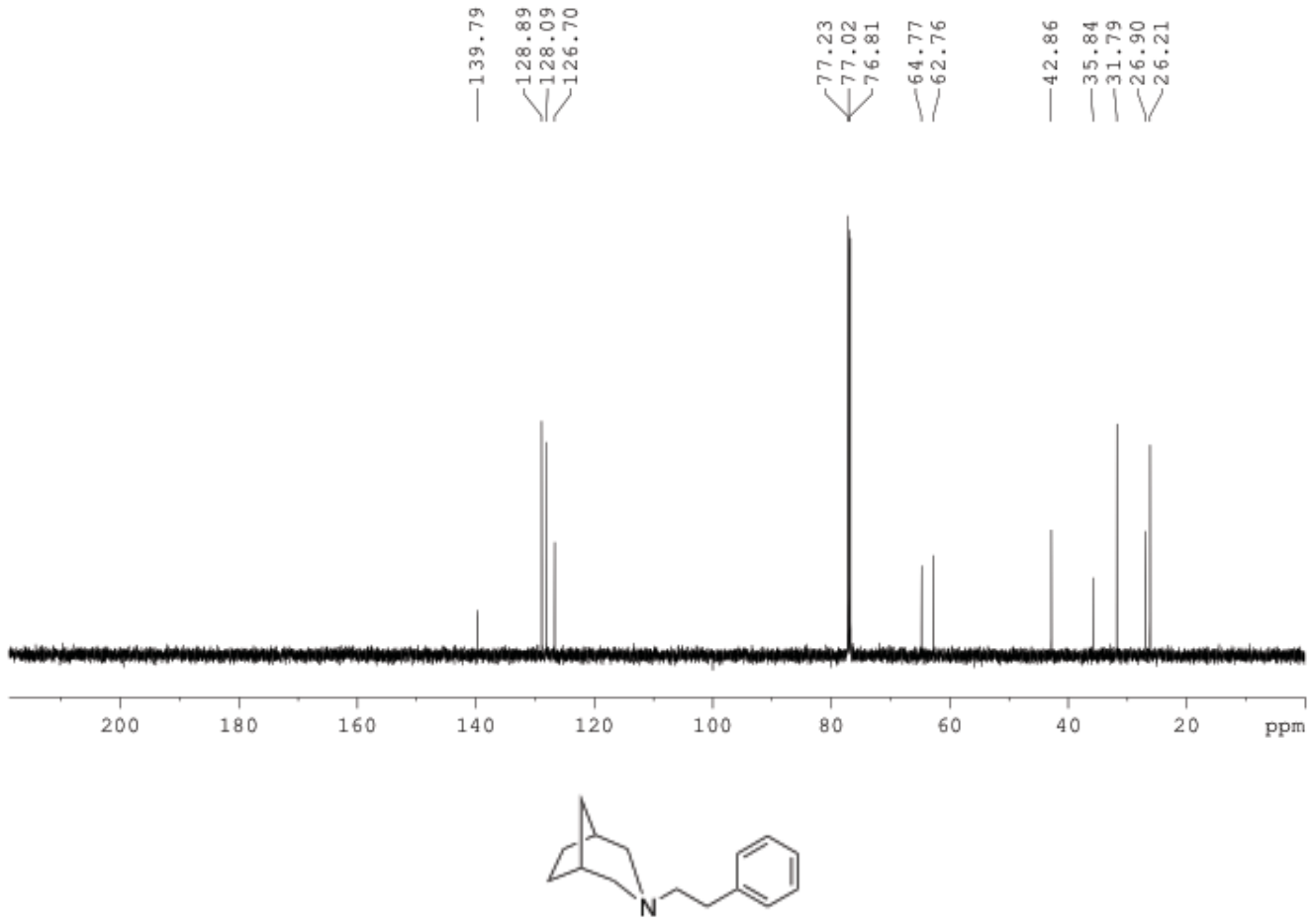

12 
1D Proton NMR

ksk02-89

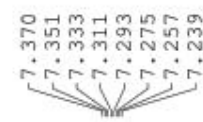

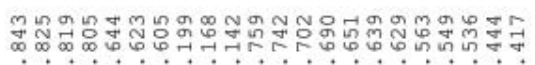

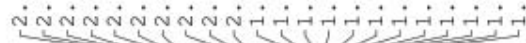

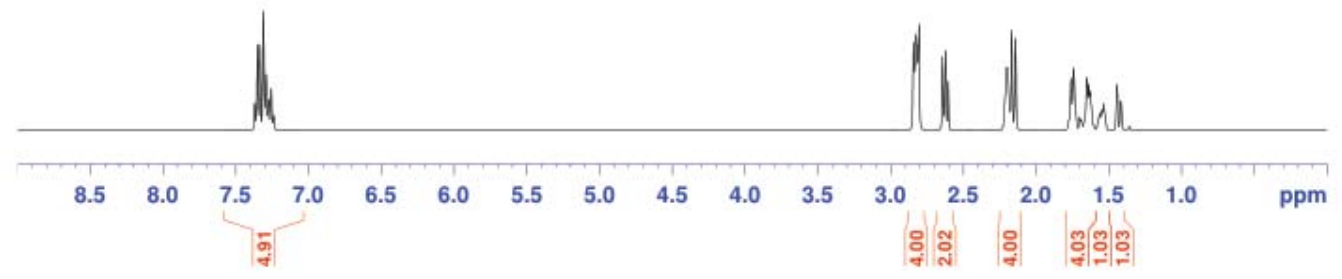

130

ksk02-89

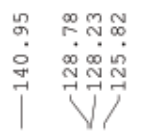

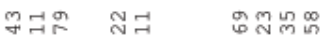

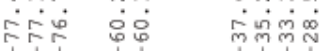

$\vee \quad \backslash / 1$
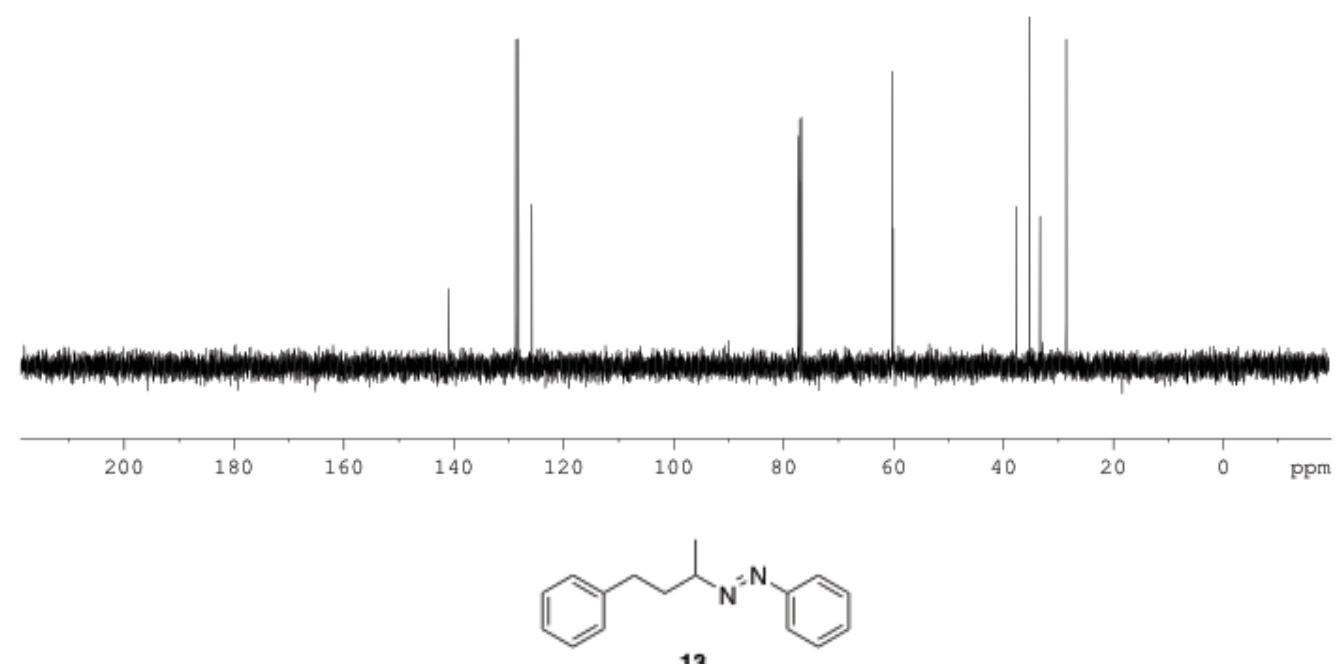
1D Proton NMR

ksk01-40

CDC13, $400 \mathrm{MHz}$

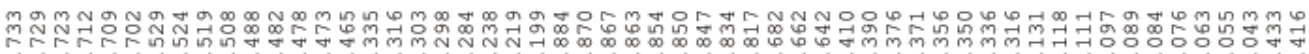
-

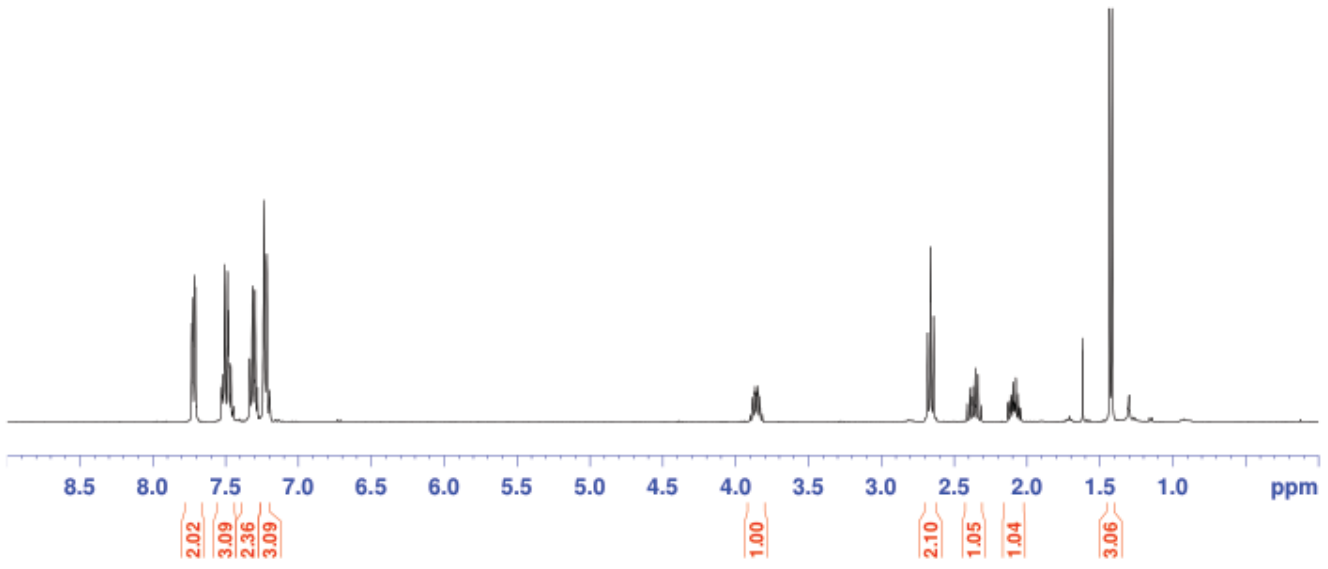

$13 \mathrm{C}$

ksk02-40

$\mathrm{CDCl} 3,400 \mathrm{MHz}$
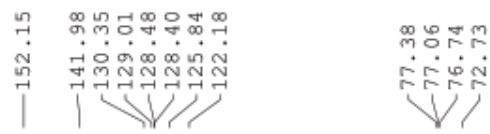

$\prod^{\infty}$

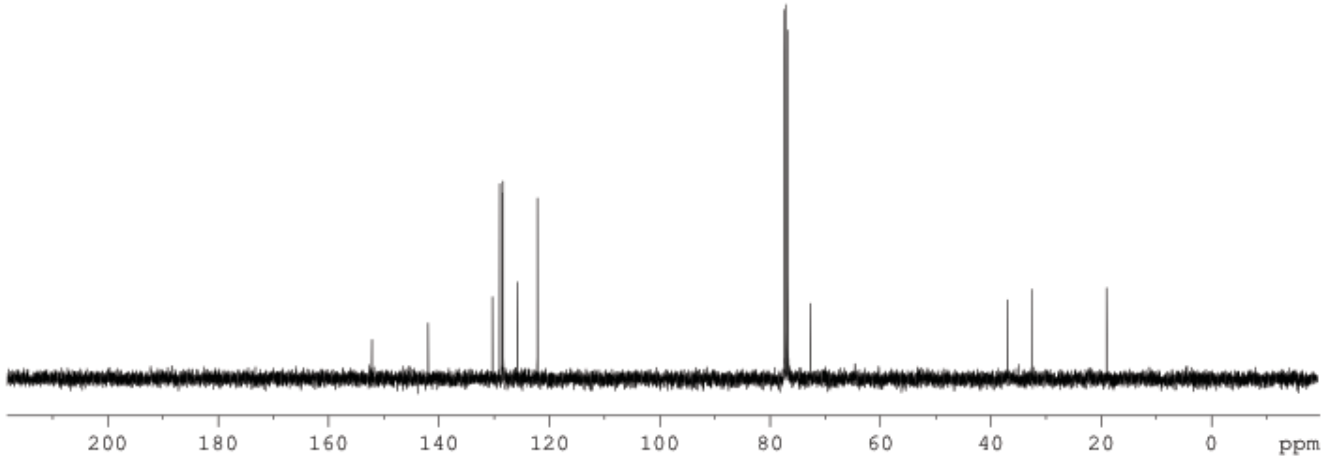

